\title{
Higher Order Large Deviation Approximations Applied to CDO Pricing
}

\author{
Laurent Veilex* \\ Initial version: December 2005; This version: February 2007
}

\begin{abstract}
We propose a Large Deviation approximation for the loss distribution of a credit portfolio and compare it as well as higher order Saddle-point and Edgeworth expansions with the standard recursion method for the pricing of CDO tranches.
\end{abstract}

\section{Introduction}

The most common approach to value synthetic CDO tranches is still via "Base Correlation" or "Local Correlation" models. Both approaches are described in [17] and [27],[2]. Those "static models" are simple extensions of the Gaussian copula, ( $\mathrm{cf} \mathrm{Li} \mathrm{[20],} \mathrm{Roncalli} \mathrm{[21]} \mathrm{).} \mathrm{As} \mathrm{the} \mathrm{value} \mathrm{of} \mathrm{a} \mathrm{CDO} \mathrm{tranche} \mathrm{is} \mathrm{the} \mathrm{sum} \mathrm{of} \mathrm{call-spreads}$ on the Loss distribution of the underlying pool, one only need to compute this loss distribution for arbitrary future times. In this framework, the loss distribution is computed via a numerical integration (cf. [23]): $L=\int L(Z) \phi(Z) d Z$ where $Z$ is Gaussian. Conditionally on $Z$, the common market factor of the model, $L=L(Z)$ is the loss distribution of a portfolio of independent names : we will focus here on the computation of this quantity using various expansion methods. We will look in particular at the higher order expansions results for the Saddle-point method and the Normal proxy, also called Jarrow- Rudd method.

The first section introduces the notations used later.

Next, The second part exploits various extensions of the Saddle-point approximation, up to the 8th order.

In the third part we expand the distribution around the Normal case : this method is similar to JarrowRudd approach, based on Edgeworth expansions of the loss distribution, but initially applied to option pricing (cf. [16])

In the fourth part, we propose a large deviation approximation based on the results of Akahira, K. Takahashi (cf. [9]).

All this numerical methods are compared with the benchmark recursion. They could be as well compared with the standard FFT method. In order to avoid numerical error, one can combine them with a Esscher transform, as described in the last Appendix. This technic prevents "aliasing" in the loss distribution computation.

In the last part, we apply those expansion formulas on a credit portfolio and compare the robustness of the methods, depending on the correlation level and seniority of the Tranches.

*email: laurentveilex@hotmail.com 


\section{Notations}

Let $n$ be the number of credit entities in the basket.

We define :

- $\tau_{i}$ : the default time of entity $i$.

- $X_{i}(t)=1_{\left(\tau_{i} \leq t\right)}$ : the default time indicator for time horizon $t$.

- $p_{i}(t)=1-\exp \left(-\int_{0}^{t} \lambda_{i}(s) d s\right)$ is the default probability up to time $t$ for name $i$ with an intensity model:

$$
p_{i}(t)=E\left(X_{i}(t)\right)
$$

- $q_{i}(t)=1-p_{i}(t)$ is the survival probability for name $i$.

- We define the counting process at time $t$ by:

$$
X(t)=\sum_{i=1}^{n} X_{i}(t) \text { with } X_{i}(t)=1_{\left\{\tau_{i} \leq t\right\}} .
$$

- $\mathcal{N}(x)$ is the CDF of the $N(0,1)$ Gaussian variable:

$$
\mathcal{N}(x)=\int_{-\infty}^{x} \phi(x) d x \text { and } \phi(x)=\frac{\exp \left(-\frac{x^{2}}{2}\right)}{\sqrt{2 \pi}}
$$

- $p_{i}^{z}(t)$ is the conditional probability on the common factor $Z=z$ (cf [23] for more details on this convention). For example, $p_{i}^{z}(t)$ can be one of the following expressions:

If we use the framework of "one factor [Gaussian] copula" or Base correlation, with correlation $z$, we have:

$$
p_{i}^{z}(t)=\mathcal{N}\left(\frac{\mathcal{N}^{-1}\left(p_{i}(t)\right)-\sqrt{\rho} z}{\sqrt{1-\rho}}\right)
$$

If we use the framework of the Local correlation (cf [27])or Random Loading Factor (cf [2]) with a correlation $z \longmapsto \rho(z)$, with values in $[0,1]$, where $z$ is $N(0,1)$, we have:

$$
p_{i}^{z}(t)=\mathcal{N}\left(\frac{\mathcal{H}^{-1}\left(p_{i}(t)\right)-\sqrt{\rho(z)} z}{\sqrt{1-\rho(z)}}\right) .
$$

We define $\mathcal{H}$ as the CDF of the variate used to correlated the default times, i.e.:

$$
\mathcal{H}(x)=P\left(U_{i}<x\right) \text { with } U_{i}=\sqrt{\rho(Z)} Z+\sqrt{1-\rho(Z)} \varepsilon_{i}
$$

with $\varepsilon_{i}$ and $Z$ are i.i.d. $N(0,1) . Z$ is the state variable. In the Gaussian framework we simply have $U_{i}=\sqrt{\rho} z+\sqrt{1-\rho} \varepsilon_{i}$. 
- $X_{i}^{z}(t)=1_{\left\{\varepsilon_{i} \leq \frac{\mathcal{H}^{-1}\left(p_{i}(t)\right)-\sqrt{\rho(z)} z}{\sqrt{1-\rho(z)}}\right\}}$ with $\varepsilon_{i} \sim N(0,1)$ i.i.d. Note that all the $X_{i}^{Z}(t)$ are independent, conditionally on $Z=z$, i.e. a particular value of the state variable.

- In that case $X^{z}(t)=\sum_{i=1}^{n} X_{i}^{z}(t)$ is the sum of independent binomial variables, with $E\left(X_{i}^{z}(t)\right)=$ $p_{i}^{z}(t)$.

$X^{z}(t)$ is the number of defaults in the basket conditional on $Z=z$ up to time $t$.

- Let $a_{i}$ be real numbers. $L_{i}^{z}(t)=\sum_{i=1}^{n} a_{i} X_{i}^{z}(t)$ is the loss accumulated at time $t$ conditional on $Z=z$. Usually $a_{i}=N_{i}\left(1-R_{i}\right)$, where $N_{i}$ is the notional invested in name $i$ (it can be negative) and $R_{i}$ is the recovery of name $i$ supposed constant here.

- The cumulants $K_{t}^{z}(\theta)$ of $X^{z}(t)$ and $L^{z}(t)$ are respectively:

$$
\begin{aligned}
& K_{t}^{z}(\theta)=\ln E\left(e^{\theta X^{z}(t)}\right)=\sum_{i=1}^{n} \ln \left(1-p_{i}^{z}(t)+p_{i}^{z}(t) e^{\theta}\right) \text { for } X^{z}(t) \\
& K_{t}^{z}(\theta)=\ln E\left(e^{\theta L^{z}(t)}\right)=\sum_{i=1}^{n} \ln \left(1-p_{i}^{z}(t)+p_{i}^{z}(t) e^{a_{i} \theta}\right) \text { for } L^{z}(t)
\end{aligned}
$$

- The notation $K^{(i)}$ means $K_{t}^{z,(i)}(\hat{\theta})$ where $\hat{\theta}$ is the Saddle-point (this will be defained in the next part).

- The expected values and variances of $X^{z}(t)$ and $L^{z}(t)$ are respectively given by:

$$
\begin{aligned}
\mu_{x} & =E\left(X^{z}(t)\right)=\Sigma p_{i}^{z}(t) \\
\mu_{l} & =E\left(L^{z}(t)\right)=\Sigma a_{i} p_{i}^{z}(t)
\end{aligned}
$$

and

$$
\begin{aligned}
& \sigma_{x}^{2}=\operatorname{Var}\left(X^{z}(t)\right)=\Sigma p_{i}^{z}(t)\left(1-p_{i}^{z}(t)\right) \\
& \sigma_{l}^{2}=\operatorname{Var}\left(X^{z}(t)\right)=\Sigma a_{i}^{2} p_{i}^{z}(t)\left(1-p_{i}^{z}(t)\right)
\end{aligned}
$$

- Some useful integrals for the Saddle-point are computed in Appendix B.

\section{Saddle-point approximations for CDO and $\mathbf{N}^{t h}$-to- defaults}

Conditionally on the state variable $Z=z$ the number of defaults in the basket at time $t$ is $X^{z}(t)=$ $\sum_{i=1}^{n} X_{i}^{z}(t)$ where the $X_{i}^{z}(t)$ are independent (cf. notations at the beginning); the Loss in the basket is $L^{z}(t)=\sum_{i=1}^{n} a_{i} X_{i}^{z}(t)$. For each approximation, we need to compute the following quantities:

- for the distribution of $X_{i}^{z}(t)$, i.e. the distribution of the number of defaults, we need to get $Q\left(X^{z}(t)=m_{0}\right)$ for each $m_{0} \in\{0,1, \ldots, n\}$;

- to compute the price of a $m_{0}^{\text {th }}$-to-default swap, we need to compute the tail of the distribution $Q\left(X^{z}(t) \geq m_{0}\right)$, for $m_{0} \in\{0,1, \ldots, n\}$ 
- to compute the price of a CDO swap we need to compute the call on loss $E\left(\left(L^{z}(t)-l_{0}\right)_{+}\right)$for different real values of $l_{0}$, either in the lower-tail (for equity tranches) or upper-tail (senior tranches).

The Saddle-point approximation method is briefly recalled below (cf. Daniels [6] and [7]) and was initially applied to portfolio credit risk ( $V A R$ and expected shortfall) in Martin et al. [25]. But the technic has been applied recently to CDO and CDO square pricing by Antonov et al. [3]. More details about this approach on a mathematical basis are available in [18].

The Edgeworth expansions consist in expending the inversion formula around the Saddle-point $\hat{\theta}$. Starting with the expansion at order 2 (i.e. the quadratic expansion and also the standard Saddle-point approximation) we extend it to the $8^{\text {th }}$ order. We compare our results with the order 4 expansion in [28].

\subsection{Quadratic Saddle-point approximation $\sim 2^{\text {nd }}$ order expansion}

\subsubsection{Computation of the density of $X^{z}(t)$}

Our aim is to apply a first order Saddle-point approximation to compute the density $Q\left(X^{z}(t)=m_{0}\right)$ for $m_{0} \in\{0,1, \ldots, n\}$. Note that [3] consider the Loss process $L$ instead of $X$. But dealing with $X$ is equivalent to deal with $L$ if we replace the quantities $a_{i}$ with 1 in the loss process. We have:

$$
Q\left(X^{z}(t)=m_{0}\right)=\frac{1}{2 i \pi} \int_{c-i \infty}^{c+i \infty} M_{t}^{z}(\theta) e^{-\theta m_{0}} d \theta
$$

where $M_{t}^{z}(\theta)=E\left[e^{\theta X^{z}(t)}\right]$ and $c>0$ is any positive number. Replacing $M_{t}^{z}(\theta)$ with $\exp \left(K_{t}^{z}(\theta)\right)$ :

$$
Q\left(X^{z}(t)=m_{0}\right)=\frac{1}{2 i \pi} \int_{c-i \infty}^{c+i \infty} e^{K_{t}^{z}(\theta)-\theta m_{0}} d \theta
$$

Let $\hat{\theta}$ be the Saddle-point, i.e. solution of $K_{t}^{z,(1)}(\hat{\theta})=m_{0}$. We define $K^{(i)}=K_{t}^{z,(i)}(\hat{\theta})$.

Note that $\hat{\theta}<0$ is $m_{0}<E\left(X^{z}(t)\right)=\sum_{i=1}^{n} p_{i}^{z}(t)$ and $\hat{\theta}>0$ otherwise. The upper-tail is the set of $m_{0}$ above the expected value of $X^{z}(t)$, i.e. such that $m_{0}>E\left(X^{z}(t)\right)$. A limited development at order 2 of the function $\theta \longmapsto K_{t}^{z}(\theta)-\theta m_{0}$ gives

$$
\begin{aligned}
K_{t}^{z}(\theta)-\theta m_{0} & =K_{t}^{z}(\hat{\theta})-\hat{\theta} m_{0}+(\theta-\hat{\theta})\left(K^{(1)}-\hat{\theta}\right)+\frac{1}{2}(\theta-\hat{\theta})^{2} K^{(2)}+o(\theta-\hat{\theta})^{2} \\
& =K_{t}^{z}(\hat{\theta})-\hat{\theta} m_{0}+\frac{1}{2}(\theta-\hat{\theta})^{2} K^{(2)}+o(\theta-\hat{\theta})^{2}
\end{aligned}
$$

then

$$
\begin{aligned}
Q\left(X^{z}(t)=m_{0}\right) & \simeq e^{K_{t}^{z}(\hat{\theta})-\hat{\theta} m_{0}} \frac{1}{2 i \pi} \int_{c-i \infty}^{c+i \infty} e^{\frac{1}{2}(\theta-\hat{\theta})^{2} K^{(2)}} d \theta \\
& \simeq e^{K_{t}^{z}(\hat{\theta})-\hat{\theta} m_{0}} J_{0}\left(K^{(2)}, \hat{\theta}\right)
\end{aligned}
$$

using the expression of $J_{0}\left(K^{(2)}, \hat{\theta}\right)$ we finally get

$$
\begin{gathered}
Q\left(X^{z}(t)=m_{0}\right) \simeq \frac{e^{K_{t}^{z}(\hat{\theta})-\hat{\theta} m_{0}}}{\sqrt{2 \pi K^{(2)}}} \\
Q\left(L^{z}(t)=l_{0}\right) \simeq \frac{e^{K_{t}^{z}(\hat{\theta})-\hat{\theta} l_{0}}}{\sqrt{2 \pi K^{(2)}}}
\end{gathered}
$$


Expressions for $K^{(1)}=K_{t}^{z,(1)}(\hat{\theta})$ and $K^{(2)}=K_{t}^{z,(2)}(\hat{\theta})$ are in Appendix-B.

So if $\sum_{i=1}^{n} p_{i}^{z}(t) 1_{\left\{p_{i}^{z}(t)>0\right\}}=m<n$ then $Q\left(X^{z}(t)=k\right)=Q\left(X^{z}(t) \geq k\right)=0$ for $k>m$.. and we don't need all this.

Note that the expression 2 is independent of $m_{0}$ or $l_{0}$ being above or below the expectation of $X^{z}(t)$ or $L^{z}(t)$, as there is no singularity in $\theta \longmapsto e^{K_{t}^{z}(\theta)-\theta m_{0}}$. This is not the case for the tail computation or the call on the Loss, as we are going to see.

\subsubsection{Computation of the survival probability $Q\left(X^{z}(t) \geq m_{0}\right)$ for the $m_{0}^{t h}$ to default event}

As before we have for $X^{z}(t)$ and $L^{z}(t)$

$$
Q\left(X^{z}(t) \geq m_{0}\right)=\frac{1}{2 i \pi} \int_{m_{0}}^{+\infty} d m \int_{c-i \infty}^{c+i \infty} M_{t}^{z}(\theta) e^{-\theta m} d \theta=\frac{1}{2 i \pi} \int_{c-i \infty}^{c+i \infty} \frac{e^{K_{t}^{z}(\theta)-\theta m_{0}}}{\theta} d \theta
$$

We have to consider 3 cases :

- If $m_{0}>E\left(X^{z}(t)\right)$ then $\hat{\theta}>0$ and we have a first order Saddle-point approximation given by

$$
\begin{aligned}
Q\left(X^{z}(t) \geq m_{0}\right) & \simeq e^{K_{t}^{z}(\hat{\theta})-\hat{\theta} m_{0}} \frac{1}{2 i \pi} \int_{c-i \infty}^{c+i \infty} \frac{e^{\frac{1}{2} K^{(2)}(\theta-\hat{\theta})^{2}}}{\theta} d \theta \\
\text { with } K_{t}^{z,(1)}(\hat{\theta}) & =m_{0}
\end{aligned}
$$

so for $m_{0} \geq E\left(X^{z}(t)\right)$ :

$$
\begin{gathered}
Q\left(X^{z}(t) \geq m_{0}\right) \simeq e^{K_{t}^{z}(\hat{\theta})-\hat{\theta} m_{0}} J_{1}\left(K^{(2)}, \hat{\theta}\right) \\
Q\left(X^{z}(t) \geq m_{0}\right) \simeq \exp \left(K_{t}^{z}(\hat{\theta})-\hat{\theta} m_{0}+\frac{1}{2} \hat{\theta}^{2} K^{(2)}\right) \mathcal{N}\left(-\hat{\theta} \sqrt{K^{(2)}}\right)
\end{gathered}
$$

- Note that if $m_{0}=E\left(X^{z}(t)\right)$ the relation is still true as the Saddle-point is at zero $(\hat{\theta}=0)$ and $K_{t}^{z}(0)=0$ so that $Q\left(X^{z}(t) \geq E\left(X^{z}(t)\right)\right)=\frac{1}{2}$.

As pointed out by Taras et al. in [28], the "Saddle-point approximation is accurate into the tail of the distribution, in fact becoming more accurate the further into the tail".

- When $m_{0}<E\left(X^{z}(t)\right)$ we have $\hat{\theta}<0$. In that case, as explained in Martin et al. [25], we need to apply the Residue Theorem to the holomorphic function $\theta \stackrel{f}{\longmapsto} \frac{e^{K_{t}^{z}(\theta)-\theta m_{0}}}{\theta}$ on the complex plane but in 0 . The theorem must be applied on the original $f$, not on the quadratic approximation $\frac{e^{\frac{1}{2} K^{(2)}(\theta-\hat{\theta})^{2}}}{\theta}$. As we have $\int_{\vec{\gamma}} f=2 i \pi \operatorname{Res}(f, 0)$ and given that:

$$
\operatorname{Res}(f, 0)=e^{K_{t}^{z}(0)}=1
$$

we can integrate on the following loop $\vec{\gamma}$ with $R>0$ :

$$
\vec{\gamma}=[\hat{\theta}+i R, \hat{\theta}-i R] \cup[\hat{\theta}-i R, c-i R] \cup[c-i R, c+i R] \cup[c+i R, \hat{\theta}+i R]
$$


as $R$ goes to infinity the only remaining terms are the integration parallel to $i \mathbb{R}$ :

$$
-\frac{1}{2 i \pi} \int_{\hat{\theta}-i \infty}^{\hat{\theta}+i \infty} \frac{e^{K_{t}^{z}(\theta)-\theta m_{0}}}{\theta} d \theta+\frac{1}{2 i \pi} \int_{c-i \infty}^{c+i \infty} \frac{e^{K_{t}^{z}(\theta)-\theta m_{0}}}{\theta} d \theta=1
$$

so finally

$$
\begin{aligned}
\frac{1}{2 i \pi} \int_{c-i \infty}^{c+i \infty} \frac{e^{K_{t}^{z}(\theta)-\theta m_{0}}}{\theta} d \theta & =1+\frac{1}{2 i \pi} \int_{\hat{\theta}-i \infty}^{\hat{\theta}+i \infty} \frac{e^{K_{t}^{z}(\theta)-\theta m_{0}}}{\theta} d \theta \\
& \simeq 1+e^{K_{t}^{z}(\hat{\theta})-\hat{\theta} m_{0}} \frac{1}{2 i \pi} \int_{\hat{\theta}-i \infty}^{\hat{\theta}+i \infty} \frac{e^{\frac{1}{2}(\theta-\hat{\theta})^{2} K^{(2)}}}{\theta} d \theta \\
& \simeq 1+e^{K_{t}^{z}(\hat{\theta})-\hat{\theta} m_{0}} J_{1}\left(K^{(2)}, \hat{\theta}\right)
\end{aligned}
$$

Using Appendix B formula we get for $\hat{\theta}<0$ (for both $X^{z}(t)$ and $\left.L^{z}(t)\right)$ :

$$
Q\left(X^{z}(t) \geq m_{0}\right) \simeq 1-\exp \left(K_{t}^{z}(\hat{\theta})-\hat{\theta} m_{0}+\frac{1}{2} \hat{\theta}^{2} K^{(2)}\right) \mathcal{N}\left(-|\hat{\theta}| \sqrt{K^{(2)}}\right)
$$

Note that the term $\exp \left(K_{t}^{z}(\hat{\theta})-\hat{\theta} m_{0}+\frac{1}{2} \hat{\theta}^{2} K^{(2)}\right)$ can sometimes explode while $\mathcal{N}\left(-|\hat{\theta}| \sqrt{K^{(2)}}\right)$ is null. For those cases $Q\left(X^{z}(t) \geq m_{0}\right)=1$.

Note also that if we are at the mean, then $\hat{\theta}=0$ so that $Q\left(X^{z}(t) \geq m_{0}\right)$. In other words, as for the Normal distribution, the Saddle-point approximation puts half of the distribution on both sides of the mean. This is obviously wrong in most of the cases when pricing CDOs.

\subsubsection{Computation of the call on the loss $E\left(L^{z}(t)-l_{0}\right)_{+}$for a CDO tranche}

We have $Q\left(L^{z}(t) \geq l_{0}\right)=\frac{1}{2 i \pi} \int_{c-i \infty}^{c+i \infty} \frac{e^{K_{t}^{z}(\theta)-\theta l_{0}}}{\theta} d \theta$. So integrating on $l_{0}$ gives:

$$
\begin{aligned}
E\left(L^{z}(t)-l_{0}\right)_{+} & =-\int_{l_{0}}^{+\infty} Q\left(L^{z}(t) \geq l\right) d l=-\int_{l_{0}}^{+\infty} \frac{1}{2 i \pi} \int_{c-i \infty}^{c+i \infty} \frac{e^{K_{t}^{z}(\theta)-\theta l}}{\theta} d \theta d l \\
& =\frac{1}{2 i \pi} \int_{c-i \infty}^{c+i \infty} \frac{e^{K_{t}^{z}(\theta)-\theta l_{0}}}{\theta^{2}} d \theta
\end{aligned}
$$

- If the strike $l_{0}$ is greater than the conditional expected loss, i.e. if $l_{0}>E^{Z}\left(L^{z}(t)\right)$ (or if $\left.\hat{\theta}>0\right)$ then, developing again $K_{t}^{z}(\theta)-\theta l_{0}$ at order 2 around the Saddle-point $\hat{\theta}$ gives the following formula, with $K_{t}^{z,(1)}(\hat{\theta})=l_{0}$ :

$$
\begin{aligned}
Q\left(L^{z}(t)-l_{0}\right)_{+} & \simeq e^{K_{t}^{z}(\hat{\theta})-\hat{\theta} l_{0}} \frac{1}{2 i \pi} \int_{c-i \infty}^{c+i \infty} \frac{e^{\frac{1}{2} K^{(2)}(\theta-\hat{\theta})^{2}}}{\theta^{2}} d \theta=e^{K_{t}^{z}(\hat{\theta})-\hat{\theta} l_{0}} J_{2}\left(K^{(2)}, \hat{\theta}\right) \\
& \simeq e^{K_{t}^{z}(\hat{\theta})-\hat{\theta} l_{0}}\left\{\sqrt{\frac{K^{(2)}}{2 \pi}}-K^{(2)} \hat{\theta} e^{\frac{1}{2} K^{(2)} \hat{\theta}^{2}} \mathcal{N}\left(-\hat{\theta} \sqrt{K^{(2)}}\right)\right\}
\end{aligned}
$$


- If the strike $l_{0}$ is smaller than the conditional expected loss, i.e. if $l_{0}<E^{Z}\left(L^{z}(t)\right)$ then, $\hat{\theta}<0$ and we have to apply the Residue Theorem as in. Let $f(\theta)=\frac{e^{K_{t}^{z}(\theta)-\theta l_{0}}}{\theta^{2}}$. Around $\theta=0$, as $K_{t}^{z}(0)=0$ and $K_{t}^{z,(1)}(0)=E^{Z}\left(L^{z}(t)\right)$ we have:

$$
\begin{aligned}
f(\theta) & \simeq \exp \left(1+K(0)+\theta\left(K_{t}^{z,(1)}(0)-l_{0}\right)+\frac{1}{2} \theta^{2} K_{t}^{z,(2)}(0)+o\left(\theta^{2}\right)\right) \\
& \simeq \frac{1+K(0)}{\theta^{2}}+\frac{E^{Z}\left(L^{z}(t)\right)-l_{0}}{\theta}+O(\theta)
\end{aligned}
$$

So the pole is $E^{Z}\left(L^{z}(t)\right)-l_{0}$ and if $c>0$ :

$$
\frac{1}{2 i \pi} \int_{c-i \infty}^{c+i \infty} \frac{e^{K_{t}^{z}(\theta)-\theta l_{0}}}{\theta^{2}} d \theta=E^{Z}\left(L^{z}(t)\right)-l_{0}
$$

and

$$
\frac{1}{2 i \pi} \int_{c-i \infty}^{c+i \infty} \frac{e^{K_{t}^{z}(\theta)-\theta l_{0}}}{\theta^{2}} d \theta=E^{Z}\left(L^{z}(t)\right)-l_{0}+\frac{1}{2 i \pi} \int_{\hat{\theta}-i \infty}^{\hat{\theta}+i \infty} \frac{e^{K_{t}^{z}(\theta)-\theta l_{0}}}{\theta^{2}} d \theta
$$

so if $\hat{\theta}<0$ :

$$
\begin{aligned}
Q\left(L^{z}(t)-l_{0}\right)_{+} & \simeq E^{Z}\left(L^{z}(t)\right)-l_{0}+e^{K_{t}^{z}(\hat{\theta})-\hat{\theta} l_{0}} J_{2}\left(K^{(2)}, \hat{\theta}\right) \\
& \simeq E^{Z}\left(L^{z}(t)\right)-l_{0}+e^{K_{t}^{z}(\hat{\theta})-\hat{\theta} l_{0}}\left\{\sqrt{\frac{K^{(2)}}{2 \pi}}-K^{(2)}|\hat{\theta}| e^{\frac{1}{2} K^{(2)} \hat{\theta}^{2}} \mathcal{N}\left(-|\hat{\theta}| \sqrt{K^{(2)}}\right)\right\}
\end{aligned}
$$

\subsection{Higher order Saddle-point approximations}

\subsubsection{Computation of the density $Q\left(X^{z}(t)=m_{0}\right) \sim 8^{t h}$ order expansion}

As mentioned in Taras et al. [28] and [9], it is possible to extent the second order approximation at higher orders, which leads to formula (6) in [28] and (2.12) in [9]. We give the formula to order 8 (cf. Appendix-F for more details)

$$
Q\left(X^{z}(t)=m_{0}\right) \simeq e^{K_{t}^{z}(\hat{\theta})-\hat{\theta} m_{0}} \times \frac{1}{\sqrt{2 \pi K^{(2)}}} \times\left\{\begin{array}{c}
1+\frac{K^{(4)}}{8 K^{(2) 2}}-\left\{\frac{K^{(6)}}{48}+\frac{5 K^{(3) 2}}{24}\right\} \frac{1}{K^{(2) 3}} \\
+\left\{\frac{K^{(8)}}{384}+\frac{35 K^{(4) 2}}{384}+\frac{7 K^{(3)} K^{(5)}}{48}\right\} \frac{1}{K^{(2) 4}}
\end{array}\right\}
$$

Note that the expansion of the exponential to order $2 k$ is equivalent to an expansion in order of $\frac{1}{K^{(2) k}}$. The odd terms in $(\theta-\hat{\theta})^{k}$ vanish for $k$ odd and the second term in $\frac{1}{K^{(2)}}$ vanishes too, because $K_{t}^{z,(1)}(\hat{\theta})=m_{0}$.

We will also compare formula (6) with Daniel's formula (we call it order 5 Taylor expansion, as it is order 6 expansion without term $\left.\frac{K^{(6)}}{48}\right)$ :

$$
Q^{\text {Daniels }}\left(X^{z}(t)=m_{0}\right) \simeq e^{K_{t}^{z}(\hat{\theta})-\hat{\theta} m_{0}} \times \frac{1}{\sqrt{2 \pi K^{(2)}}} \times\left\{1+\frac{K^{(4)}}{8 K^{(2) 2}}-\frac{5 K^{(3) 2}}{24 K^{(2) 3}}\right\}
$$




\subsubsection{Computation of the tail $Q\left(X^{z}(t) \geq m_{0}\right) \sim 4^{t h}$ and $6^{\text {th }}$ order expansion}

The tail approximation for an expansion of $(\theta-\hat{\theta})$ at $4^{\text {th }}$ and $6^{\text {th }}$ order is given by $Q^{4^{t h}}\left(X^{z}(t) \geq m_{0}\right)$ and $Q^{6^{t h}}\left(X^{z}(t) \geq m_{0}\right)$ :

$$
\begin{aligned}
& Q^{4^{t h}}\left(X^{z}(t) \geq m_{0}\right) \simeq 1_{\{\hat{\theta} \leq 0\}} \\
& +\operatorname{sign}(\hat{\theta}) e^{K_{t}^{z}(\hat{\theta})-\hat{\theta} m_{0}} e^{\frac{1}{2} K^{(2)} \hat{\theta}^{2}} \mathcal{N}\left(-\sqrt{K^{(2)}}|\hat{\theta}|\right)\left(1-\frac{K^{(3)} \hat{\theta}^{3}}{6}+\frac{K^{(4)} \hat{\theta}^{4}}{24}\right) \\
& +\frac{e^{K_{t}^{z}(\hat{\theta})-\hat{\theta} m_{0}}}{24 \sqrt{2 \pi} K^{(2) \frac{3}{2}}}\left(1-\hat{\theta}^{2} K^{(2)}\right)\left(\hat{\theta} K^{(4)}-4 K^{(3)}\right)
\end{aligned}
$$

The details of the computations are given in Appendix-F. Note that our results are different from Taras [28] .

The $6^{\text {th }}$ order is given by:

$$
\begin{aligned}
& Q^{6^{t h}}\left(X^{z}(t) \geq m_{0}\right) \\
\simeq & 1_{\{\hat{\theta} \leq 0\}}+\operatorname{sign}(\hat{\theta}) e^{K_{t}^{z}(\hat{\theta})-\hat{\theta} m_{0}} \times e^{\frac{1}{2} K^{(2)} \hat{\theta}^{2}} \mathcal{N}\left(-\sqrt{K^{(2)}}|\hat{\theta}|\right) \times \\
& \left\{1-\frac{K^{(3)} \hat{\theta}^{3}}{6}+\frac{K^{(4)} \hat{\theta}^{4}}{24}-\frac{K^{(5)} \hat{\theta}^{5}}{120}+\frac{K^{(6)} \hat{\theta}^{6}}{720}+\frac{K^{(3) 2} \hat{\theta}^{6}}{72}\right\} \\
+ & \frac{e^{K_{t}^{z}(\hat{\theta})-\hat{\theta} m_{0}}}{72 \sqrt{2 \pi} K^{(2) \frac{5}{2}}} \times\left\{\begin{array}{c}
3 K^{(2)}\left(1-\hat{\theta}^{2} K^{(2)}\right)\left[\hat{\theta} K^{(4)}-4 K^{(3)}+\frac{\hat{\theta}^{2}}{5}\left(\frac{\hat{\theta} K^{(6)}}{6}-K^{(5)}\right)\right] \\
-\hat{\theta} K^{(3) 2} \cdot\left(18-\hat{\theta}^{2} K^{(2)}+\hat{\theta}^{4} K^{(2) 2}\right) \\
+\frac{9 K^{(5)}}{5}+K^{(6)}\left(\frac{3}{2}-\frac{9 \hat{\theta}}{5}\right)+15 K^{(3) 2}
\end{array}\right\}
\end{aligned}
$$

We recall Lugannani \& Rice formula for the tail :

$$
\begin{aligned}
Q^{\text {Lug.\&Rce }}\left(X^{z}(t) \geq m_{0}\right) & =1-\mathcal{N}\left(\operatorname{sign}(\hat{\theta}) \sqrt{2 .\left|K_{t}^{z}(\hat{\theta})-\hat{\theta} m_{0}\right|}\right) \\
& +\quad \frac{e^{K_{t}^{z}(\hat{\theta})-\hat{\theta} m_{0}}}{\sqrt{2 \pi}} \times\left\{\frac{1}{\hat{\theta} \sqrt{K^{(2)}}}-\frac{1}{\operatorname{sign}(\hat{\theta}) \sqrt{2 \cdot\left|K_{t}^{z}(\hat{\theta})-\hat{\theta} m_{0}\right|}}\right\}
\end{aligned}
$$


and Damian Taras, Christopher Cloke-Browne and Evan Kalimtgis formula:

$$
\begin{aligned}
& Q^{T C B K}\left(X^{z}(t) \geq m_{0}\right) \\
\simeq \quad & 1_{\{\hat{\theta} \leq 0\}}+\operatorname{sign}(\hat{\theta}) e^{K_{t}^{z}(\hat{\theta})-\hat{\theta} m_{0}} \times e^{\frac{1}{2} K^{(2)} \hat{\theta}^{2}} \mathcal{N}\left(-\sqrt{K^{(2)}}|\hat{\theta}|\right) \times \\
& \left\{1-\frac{K^{(3)} \hat{\theta}^{3}}{6}+\frac{K^{(4)} \hat{\theta}^{4}}{24}+\frac{K^{(3) 2} \hat{\theta}^{6}}{72}\right\} \\
& +\frac{e^{K_{t}^{z}(\hat{\theta})-\hat{\theta} m_{0}}}{72 \sqrt{2 \pi} K^{(2) \frac{5}{2}}} \times\left\{\begin{array}{c}
3 K^{(2)}\left(1-\hat{\theta}^{2} K^{(2)}\right)\left(\hat{\theta} K^{(4)}-4 K^{(3)}\right) \\
-\hat{\theta} K^{(3) 2} \cdot\left(3-\hat{\theta}^{2} K^{(2)}+\hat{\theta}^{4} K^{(2) 2}\right)
\end{array}\right\}
\end{aligned}
$$

3.2.3 Computation of the call on the loss $E\left(L^{z}(t)-l_{0}\right)_{+} \sim 4^{t h}$ and $6^{\text {th }}$ order expansion

The details of the following formula are given in Appendix-F :

$$
E\left(L^{z}(t)-l_{0}\right)_{+} \simeq 1_{\{\hat{\theta} \leq 0\}} \cdot\left(E^{Z}\left(L^{z}(t)\right)-l_{0}\right)+e^{K_{t}^{z}(\hat{\theta})-\hat{\theta} l_{0}} \times S^{4 t h}
$$

with:

$$
\begin{aligned}
S^{4 t h}= & \hat{\theta}^{2} \operatorname{sign}(\hat{\theta}) \mathcal{N}\left(-\sqrt{K^{(2)}}|\hat{\theta}|\right) e^{\frac{1}{2} K^{(2)} \hat{\theta}^{2}}\left\{\frac{K^{(3)}}{2}-\frac{K^{(4)} \hat{\theta}}{6}\right\} \\
& -|\hat{\theta}| K^{(2)} \mathcal{N}\left(-\sqrt{K^{(2)}}|\hat{\theta}|\right) e^{\frac{1}{2} K^{(2)} \hat{\theta}^{2}}\left\{1-\frac{K^{(3)} \hat{\theta}^{3}}{6}+\frac{K^{(4)} \hat{\theta}^{4}}{24}\right\} \\
& +\frac{1}{\sqrt{2 \pi} K^{(2) \frac{3}{2}}}\left\{K^{(2) 2}-\frac{K^{(4)}}{24}+K^{(2)} \hat{\theta}\left(-\frac{K^{(3)}}{3}+\frac{K^{(4)} \hat{\theta}}{8}-\frac{K^{(2)} K^{(3)} \hat{\theta}^{2}}{6}+\frac{K^{(2)} K^{(4)} \hat{\theta}^{3}}{24}\right)\right\}
\end{aligned}
$$

and the $6^{\text {th }}$ order:

$$
E\left(L^{z}(t)-l_{0}\right)_{+} \simeq 1_{\{\hat{\theta} \leq 0\}} \cdot\left(E^{Z}\left(L^{z}(t)\right)-l_{0}\right)+e^{K_{t}^{z}(\hat{\theta})-\hat{\theta} l_{0}} \times S^{6 t h}
$$

with:

$$
\begin{aligned}
S^{6 t h}= & \hat{\theta}^{2} \operatorname{sign}(\hat{\theta}) \mathcal{N}\left(-\sqrt{K^{(2)}}|\hat{\theta}|\right) e^{\frac{1}{2} K^{(2)} \hat{\theta}^{2}}\left\{\frac{K^{(3)}}{2}-\frac{K^{(4)} \hat{\theta}}{6}+\frac{K^{(5)} \hat{\theta}^{2}}{24}-\frac{K^{(6)} \hat{\theta}^{3}}{120}-\frac{K^{(3) 2} \hat{\theta}^{3}}{12}\right\} \\
& -|\hat{\theta}| K^{(2)} \mathcal{N}\left(-\sqrt{K^{(2)}}|\hat{\theta}|\right) e^{\frac{1}{2} K^{(2)} \hat{\theta}^{2}}\left\{1-\frac{K^{(3)} \hat{\theta}^{3}}{6}+\frac{K^{(4)} \hat{\theta}^{4}}{24}-\frac{K^{(5)} \hat{\theta}^{5}}{120}+\frac{K^{(6)} \hat{\theta}^{6}}{720}+\frac{K^{(3) 2} \hat{\theta}^{6}}{72}\right\} \\
& +\frac{1}{\sqrt{2 \pi} K^{(2) \frac{5}{2}}}\left\{\begin{array}{c}
K^{(2) 2} \hat{\theta}\left(-\frac{K^{(3)}}{3}+\frac{K^{(4)} \hat{\theta}}{8}-\frac{K^{(5)} \hat{\theta}^{2}}{30}+\frac{K^{(6)} \hat{\theta}^{3}}{144}+\frac{5 K^{(3) 2} \hat{\theta}^{3}}{72}\right) \\
+K^{(2)}\left(-\frac{K^{(4)}}{24}+\frac{K^{(5)} \hat{\theta}}{60}-\frac{K^{(6)} \hat{\theta}^{2}}{240}-\frac{K^{(3) 2} \hat{\theta}^{2}}{24}\right) \\
+K^{(2) 3}\left(1-\frac{K^{(3)} \hat{\theta}^{3}}{6}+\frac{K^{(4)} \hat{\theta}^{4}}{24}-\frac{K^{(5)} \hat{\theta}^{5}}{120}+\frac{K^{(6)} \hat{\theta}^{6}}{720}+\frac{K^{(3) 2} \hat{\theta}^{6}}{72}\right) \\
+\frac{K^{(6)}}{240}+\frac{K^{(3) 2}}{24}
\end{array}\right\}
\end{aligned}
$$

Note that $K^{(i)}=K_{t}^{z,(i)}(\hat{\theta})$ where $\hat{\theta}$ is the Saddle-point, i.e. solution of $K_{t}^{z,(1)}(\hat{\theta})=l_{0}$. 


\section{The Normal-Proxy approximation of David Shelton}

The approach from David Shelton [26] is an even more direct and efficient approximation than the Saddlepoint. All it needs, conditional on the variable $Z$, is : the value of the expectation of $X^{z}(t)$ and its variance (cf. the notations at the beginning of this paper). We have $\mu_{x}=\Sigma p_{i}^{z}(t)$ and $\sigma_{x}^{2}=\Sigma p_{i}^{z}(t)\left(1-p_{i}^{z}(t)\right)$ and we assume that the distribution of $X^{z}(t)$ is Normal $N\left(\mu_{x}, \sigma_{x}\right)$. This approximation is particulary good for large portfolio as it is somewhat a limit of the theorem of large numbers. The most useful property of this approximation is that given a value of $z$ the density computed with the normal-proxy is generally very different from the theoretical one, but when we integrate numerically on $z$ then it becomes very close to the real distribution (cf. numerical results).

The conditional density of $X^{z}(t)$ is simply given by

$$
Q_{N P}\left(X^{z}(t)=m_{0}\right)=\frac{1}{\sqrt{2 \pi} \sigma_{x}} \exp \left(-\frac{\left(m_{0}-\mu_{x}\right)^{2}}{2 \sigma_{x}}\right)
$$

and a call on Loss by

$$
\begin{aligned}
E\left(L^{z}(t)-K\right)_{+} & =\sigma_{l}\{\phi(\tilde{K})-\tilde{K} \mathcal{N}(-\tilde{K})\} \\
\tilde{K} & =\frac{K-\mu_{l}}{\sigma_{l}} \\
\mu_{l} & =\Sigma a_{i} p_{i}^{z}(t) \\
\sigma_{l}^{2} & =\Sigma a_{i}^{2} p_{i}^{z}(t)\left(1-p_{i}^{z}(t)\right)
\end{aligned}
$$

Note that the density, tail and call should not be renormalized with $\mathcal{N}\left(\frac{X_{\max }^{z}-\mu_{x}}{\sigma_{x}}\right)-\mathcal{N}\left(\frac{X_{\min }^{z}-\mu_{x}}{\sigma_{x}}\right)$ to make sure that the density sum to one.

\section{Expanding the Normal Proxy : the Jarrow-Rudd approach}

As we will see in the numerical illustrations, the Normal-Proxy is very efficient in most cases, but not for very thin or senior tranches. Our aim here is to refine it by capturing higher order moments of the distribution. The idea is to start from a given distribution (i.e. we start from the Normal distribution) and approximate the real distribution of the loss using higher moments : the skew and the kurtosis. This is called a generalized Edgeworth series expansion of the density (cf. [5], [19],[16]). From the expansion of the density as in [16], we have directly the expansion of the call on loss.

\subsection{Computation of the density using Jarrow-Rudd expansion}

As in [16] we define $x \longmapsto a(x)$ as the approximate density (the Normal one, cf. (9)) and $x \longmapsto f(x)$ as the real density of $L^{z}(t)$ that we want to expand.

Following Jarrow-Rudd expansion (4) in [16], we have:

$$
\begin{aligned}
f(x) \approx & a(x)+\frac{\left(K_{2}(f)-K_{2}(a)\right)}{2} a^{(2)}(x)-\frac{\left(K_{3}(f)-K_{3}(a)\right)}{6} a^{(3)}(x) \\
& +\frac{\left(K_{4}(f)-K_{4}(a)\right)+3\left(K_{2}(f)-K_{2}(a)\right)^{2}}{24} a^{(4)}(x)
\end{aligned}
$$


with $K_{i}(f)=K^{(i)}(\hat{\theta})$ is the cumulant of order $i$ for the density $f$, taken at value $\hat{\theta}=0 . a^{(i)}(x)$ is the derivative of order $i$. In the paper of Jarrow-Rudd, The value of $\hat{\theta}$ is zero (there is no Saddle-point approximation here) . The formula above is proven in [16]. The idea is to write the Taylor series of the first cumulant of $f$ i.e. $K_{0}(f)(\theta)$ around $\theta=0$ and to do the same with $K_{0}(a)(\theta)$. Taking the difference of those series up to a order $N$ one have $K_{0}(f)(\theta) \approx K_{0}(a)(\theta)+\sum_{i=1}^{N}\left(K_{i}(f)-K_{i}(a)\right) \frac{\theta^{i}}{i !}$. Then taking the exponential of this equation, one find a relation between the characteristic functions of $f$ and $a: M_{0}(f) \approx$ $M_{0}(a) \exp \left(\sum_{i=1}^{N}\left(K_{i}(f)-K_{i}(a)\right) \frac{\theta^{i}}{i !}\right)$. Again, we do a Taylor expansion of the exponential to finally have $\exp \left(\sum_{i=1}^{N}\left(K_{i}(f)-K_{i}(a)\right) \frac{\theta^{i}}{i !}\right) \approx \sum_{i=1}^{N} E_{j} \frac{\theta^{i}}{i !}$. This step is actually very similar to the computation of expansions in the Saddle-point framework.

Using the inverse Fourier transform of this series one finally find a relationship between the density of $f$ and the density of $a$

Let define by $\mu_{l}$ and $\sigma_{l}^{2}$ respectively the mean and the variance of the loss $L^{z}(t)$. Then concerning $a(x)$,we need to have $K_{1}(a)=K_{1}(f)=\mu_{l}$. We use $a(x)$ given by the normal proxy. We know that it is already a good approximation of the real density :

$$
a(x)=\frac{1}{\sqrt{2 \pi} \sigma_{l}} \exp \left(-\frac{\left(x-\mu_{l}\right)^{2}}{2 \sigma_{l}^{2}}\right)
$$

In particular, we have

$$
\begin{aligned}
K_{2}(a) & =\sigma_{l}^{2}=K_{2}(f) \\
K_{i}(a) & =0 \text { for all } i \geq 3
\end{aligned}
$$

The formula for $K_{i}(f)$ when $f$ is the density of the loss process $L^{z}(t)$ are given in Appendix A. So we have at order 4 :

$$
f(x) \approx a(x)-\frac{K_{3}(f)}{6} a^{(3)}(x)+\frac{K_{4}(f)}{24} a^{(4)}(x)
$$

Note that because the first two moments of $f$ and $a$ are chosen to be equal, there is not weight on $a^{(1)}(x)$ and $a^{(2)}(x)$. This formula, because it shows the expansion of the density, is much more instructive and explicit than the Saddle-point approximation. One can see how the real density differs from the normal density by looking at the weights on higher order terms, i.e. skew and kurtosis. Indeed, the term in front of $a^{(2)}(x)$ is a function of the difference in variances. If $L$ was normal, with a different volatility than that of $a$ then we would have $f(x) \approx a(x)+\frac{\left(\sigma_{f}-\sigma_{l}^{2}\right)}{2} a^{(2)}(x)$. The term in front of $a^{(3)}(x)$ captures the skewness of $f$ and the last one the kurtosis.

The expansion (10) can be decomposed into a polynomial $P(\tilde{x})$ multiplied with $\phi(\tilde{x})$ :

$$
\begin{aligned}
f(x) & \approx P(\tilde{x}) \frac{1}{\sigma} \phi(\tilde{x}) \\
\tilde{x} & =\frac{x-\mu}{\sigma}
\end{aligned}
$$

\subsubsection{Order 3 expansion}

We have $f(x) \approx a(x)-\frac{K_{3}(f)}{6} a^{(3)}(x)$ so:

$$
P(\tilde{x})=1-\frac{K_{3}}{2 \sigma^{3}} \tilde{x}+\frac{K_{3}}{6 \sigma^{3}} \tilde{x}^{3}
$$




\subsubsection{Order 4 expansion:}

We have $f(x) \approx a(x)-\frac{K_{3}(f)}{6} a^{(3)}(x)+\frac{K_{4}(f)}{24} a^{(4)}(x)$ so:

$$
P(\tilde{x})=1+\frac{K_{4}}{8 \sigma^{4}}-\frac{K_{3}}{2 \sigma^{3}} \tilde{x}-\frac{K_{4}}{4 \sigma^{4}} \tilde{x}^{2}+\frac{K_{3}}{6 \sigma^{3}} \tilde{x}^{3}+\frac{K_{4}}{24 \sigma^{4}} \tilde{x}^{4}
$$

with $K_{i}$ either the cumulants of $X^{z}(t)$ or $L^{z}(t)$ computed in Appendix C (note that in appendix C, we compute the cumulants associated with an Esscher transform : here the cumulants $K_{i}$ are computed with $\hat{\theta}=0)$. Mean $\mu$ and volatility $\sigma$ are those of $X^{z}(t)$ or $L^{z}(t)$

\subsection{Computation of the call on Loss using Jarrow-Rudd expansion}

Now that we have an explicit expansion of the density we can easily compute $E\left(L^{z}(t)-K\right)_{+}$from expression (10) :

$$
E\left(L^{z}(t)-K\right)_{+}=\sum_{i=0}^{4} \eta_{i} \int_{\tilde{K}}^{+\infty}(z-\tilde{K}) z^{i} \phi(z) d z
$$

with $\tilde{K}=\frac{x-\mu_{l}}{\sigma_{l}}$ and $\eta_{i}$ the coefficient of degree $i$ of the polynomial $P$ in (11).

Using Appendix $\mathrm{C}$ formulas of the moments of a Normal variable stuck at $\tilde{K}$ we find:

\subsubsection{Order 3 expansion:}

We have $P(x)=1-\frac{K_{3}}{2 \sigma^{3}} x+\frac{K_{3}}{6 \sigma^{3}} x^{3}$ so

$$
\begin{aligned}
E\left(L^{z}(t)-K\right)_{+} & =\sigma_{l}\left\{\left(1+\frac{K_{3}}{6 \sigma_{l}^{3}} \tilde{K}\right) \phi(\tilde{K})-\tilde{K} \mathcal{N}(-\tilde{K})\right\} \\
& =E^{\operatorname{Proxy}}\left(L^{z}(t)-K\right)_{+}+\frac{K_{3}}{6 \sigma_{l}^{2}} \tilde{K} \phi(\tilde{K})
\end{aligned}
$$

\subsubsection{Order 4 expansion:}

We have $P(x)=1+\frac{K_{4}}{8 \sigma^{4}}-\frac{K_{3}}{2 \sigma^{3}} x-\frac{K_{4}}{4 \sigma^{4}} x^{2}+\frac{K_{3}}{6 \sigma^{3}} x^{3}+\frac{K_{4}}{24 \sigma^{4}} x^{4}$ so

$$
\begin{aligned}
E\left(L^{z}(t)-K\right)_{+} & =\sigma_{l}\left\{\left(1-\frac{K_{4}}{24 \sigma_{l}^{4}}+\frac{K_{3}}{6 \sigma_{l}^{3}} \tilde{K}+\frac{5 K_{4}}{24 \sigma_{l}^{4}} \tilde{K}^{2}\right) \phi(\tilde{K})-\tilde{K} \mathcal{N}(-\tilde{K})\right\} \\
& =E^{\operatorname{Proxy}}\left(L^{z}(t)-K\right)_{+}+\left(\frac{K_{3}}{6 \sigma_{l}^{2}} \tilde{K}+\frac{5 K_{4}}{24 \sigma_{l}^{3}} \tilde{K}^{2}-\frac{K_{4}}{24 \sigma_{l}^{3}}\right) \phi(\tilde{K})
\end{aligned}
$$

\section{Higher order Large Deviation approximations}

\subsection{Computation of the density $Q\left(X^{z}(t)=m_{0}\right)$}

The recursion algorithm in Akahira \& Takahashi [9] enables to relate explicitly density $Q\left(X^{z}(t)=m_{0}\right)$ and $Q\left(X^{z}(t)=m_{0}+k\right)$ for any $k$. 
This can be applied to can be applied to $X^{z}(t)$ or $L^{z}(t)$. The only thing we need is the value of the cumulants. Let suppose you know $Q\left(X^{z}(t)=m_{0}\right)$. We want to compute $Q\left(X^{z}(t)=m_{0}+k\right)$. Akahira, $\mathrm{K}$. Takahashi propose Daniel's formula for the initial value at $k=0$ :

$$
Q\left(X^{z}(t)=m_{0}\right) \simeq \frac{e^{K_{t}^{z}(\hat{\theta})-\hat{\theta} m_{0}}}{\sqrt{2 \pi K^{(2)}}}\left\{1+\frac{K^{(4)}}{8 K^{(2) 2}}-\frac{5 K^{(3) 2}}{24 K^{(2) 3}}\right\}
$$

Then the result of Akahira \& Takahashi is the tail approximation, $\hat{\theta}$ being the Saddle-point at $m_{0}$ :

- if $m_{0} \geq E\left(X^{z}(t)\right)$ :

$$
Q\left(X^{z}(t)=m_{0}+k\right)=Q\left(X^{z}(t)=m_{0}\right) \exp \left(-k\left(\hat{\theta}+\frac{K^{(3)}(\hat{\theta})}{2 K^{(2)}(\hat{\theta})^{2}}\right)-\frac{k^{2}}{2 K^{(2)}(\hat{\theta})}+O\left(\frac{1}{n^{2}}\right)\right)
$$

- and for $m_{0}<E\left(X^{z}(t)\right)$ :

$$
Q\left(X^{z}(t)=m_{0}-k\right)=Q\left(X^{z}(t)=m_{0}\right) \exp \left(k\left(\hat{\theta}+\frac{K^{(3)}(\hat{\theta})}{2 K^{(2)}(\hat{\theta})^{2}}\right)-\frac{k^{2}}{2 K^{(2)}(\hat{\theta})}+O\left(\frac{1}{n^{2}}\right)\right)
$$

We extend the result of Akahira et al. to take into account higher order powers in $k$.

- if $m_{0} \geq E\left(X^{z}(t)\right)$ :

$$
Q\left(X^{z}(t)=m_{0}+k\right)=\ldots
$$

- and for $m_{0}<E\left(X^{z}(t)\right)$ :

$$
Q\left(X^{z}(t)=m_{0}-k\right)=\ldots
$$

The proof is given in appendix $\mathrm{G}$.

\subsection{Computation of the tail $Q\left(m_{0} \geq E\left(X^{z}(t)\right)\right)$}

- In that case, we get the tail as $Q\left(X^{z}(t) \geq m_{0}\right)=1-Q\left(X^{z}(t) \leq m_{0}-1\right)$, so Saddle-point $\hat{\theta}$ should be carefully computed at $m_{0}-1$ instead of $m_{0}$.

- if $m_{0} \geq E\left(X^{z}(t)\right)$ :

$$
Q\left(X^{z}(t) \geq m_{0}\right) \approx Q\left(X^{z}(t)=m_{0}\right) \sum_{k=0}^{n-m_{0}} \exp \left(-k\left(\hat{\theta}+\frac{K^{(3)}}{2 K^{(2) 2}}\right)-\frac{k^{2}}{2 K^{(2)}}\right)
$$

- and for $m_{0}<E\left(X^{z}(t)\right)$ :

$$
Q\left(X^{z}(t) \leq m_{0}\right) \approx Q\left(X^{z}(t)=m_{0}\right) \sum_{k=0}^{m_{0}} \exp \left(k\left(\hat{\theta}+\frac{K^{(3)}}{2 K^{(2) 2}}\right)-\frac{k^{2}}{2 K^{(2)}}\right)
$$


We can see in the idea of the proof that as opposed to the Saddle-point approximation for the tail $Q\left(X^{z}(t) \geq m_{0}\right)$, the Large deviation approximation basically uses the Saddle-point information at all points $Q\left(X^{z}(t)=m_{0}+k\right)$ and not only at $m_{0}$. The approximation for the tail $Q\left(X^{z}(t) \geq m_{0}\right)$ is

consequently more accurate than for the Saddle-point, which in fact diverge if we use higher orders.

When $m_{0}<E\left(X^{z}(t)\right)$ we get the upper tail via the lower tail : $Q\left(X^{z}(t) \geq m_{0}\right)=1-Q\left(X^{z}(t) \leq m_{0}-1\right)$.

\subsection{Computation of the call on loss $E\left(L^{z}(t)-l_{0}\right)_{+}$}

The computation of the call on loss $E\left(L^{z}(t)-l_{0}\right)_{+}$is straightforward. We have to consider 2 cases:

- If $l \geq E\left(L^{z}(t)\right)$ and $\hat{\theta}$ being the Saddle-point at $l_{0}$ :

$$
E\left(L^{z}(t)-l_{0}\right)_{+}=Q\left(L^{z}(t)=l_{0}\right) \sum_{k=0}^{n-l_{0}} k \cdot \exp \left(-k\left(\hat{\theta}+\frac{K^{(3)}}{2 K^{(2) 2}}\right)-\frac{k^{2}}{2 K^{(2)}}\right)
$$

- if $l_{0}<E\left(L^{z}(t)\right)$ : In that case, we compute the Saddle-point $\hat{\theta}$ at $\mu_{l}=E\left(L^{z}(t)\right)$ and we cut the integral in 2 parts :

$$
\begin{aligned}
I_{1} & =Q\left(L^{z}(t)=\mu_{l}\right) \sum_{k=0}^{n-\mu_{l}}\left(\mu_{l}+k-l_{0}\right) \cdot \exp \left(-k\left(\hat{\theta}+\frac{K^{(3)}}{2 K^{(2) 2}}\right)-\frac{k^{2}}{2 K^{(2)}}\right) \\
I_{2} & =Q\left(L^{z}(t)=\mu_{l}\right) \sum_{k=1}^{\mu_{l}-l_{0}}\left(\mu_{l}-k-l_{0}\right) \cdot \exp \left(k\left(\hat{\theta}+\frac{K^{(3)}}{2 K^{(2) 2}}\right)-\frac{k^{2}}{2 K^{(2)}}\right) \\
E\left(L^{z}(t)-l_{0}\right)_{+} & =I_{1}+I_{2}
\end{aligned}
$$

\section{Numerical results}

We consider an homogeneous portfolio of 100 names. If the default intensity is sufficiently large, to highlight the differences in the distribution we obtain (intensity is $1000 \mathrm{bps}$ ) : 


\begin{tabular}{|c|c|c|c|c|c|}
\hline \# default & recursion & saddle point & Large Deviation & Normal proxy & Jarow Rudd \\
\hline 13 & 0.06217 & 0.06196 & 0.06070 & 0.06726 & 0.06134 \\
14 & 0.04063 & 0.04048 & 0.04000 & 0.04235 & 0.04031 \\
15 & 0.02450 & 0.02440 & 0.02424 & 0.02374 & 0.02471 \\
16 & 0.01369 & 0.01363 & 0.01359 & 0.01185 & 0.01410 \\
17 & 0.00711 & 0.00708 & 0.00708 & 0.00527 & 0.00742 \\
18 & 0.00345 & 0.00343 & 0.00344 & 0.00208 & 0.00356 \\
19 & 0.00157 & 0.00156 & 0.00156 & 0.00073 & 0.00154 \\
20 & 0.00067 & 0.00066 & 0.00067 & 0.00023 & 0.00060 \\
21 & 0.00027 & 0.00027 & 0.00027 & 0.00006 & 0.00021 \\
22 & 0.00010 & 0.00010 & 0.00010 & 0.00002 & 0.00006 \\
23 & 0.00004 & 0.00004 & 0.00004 & $\mathbf{0 . 0 0 0 0 0}$ & $\mathbf{0 . 0 0 0 0 2}$ \\
24 & 0.00001 & 0.00001 & 0.00001 & $\mathbf{0 . 0 0 0 0 0}$ & $\mathbf{0 . 0 0 0 0 0}$ \\
25 & 0.00000 & 0.00000 & 0.00000 & $\mathbf{0 . 0 0 0 0 0}$ & $\mathbf{0 . 0 0 0 0 0}$ \\
26 & 0.00000 & 0.00000 & 0.00000 & $\mathbf{0 . 0 0 0 0 0}$ & $\mathbf{0 . 0 0 0 0 0}$ \\
27 & 0.00000 & 0.00000 & 0.00000 & 0.00000 & 0.00000 \\
28 & 0.00000 & 0.00000 & 0.00000 & 0.00000 & 0.00000 \\
\hline
\end{tabular}

Comparison of loss distributions based on different tails approximations

The densities are very close to each other. The distribution is plotted for the number of defaults in [13,22].

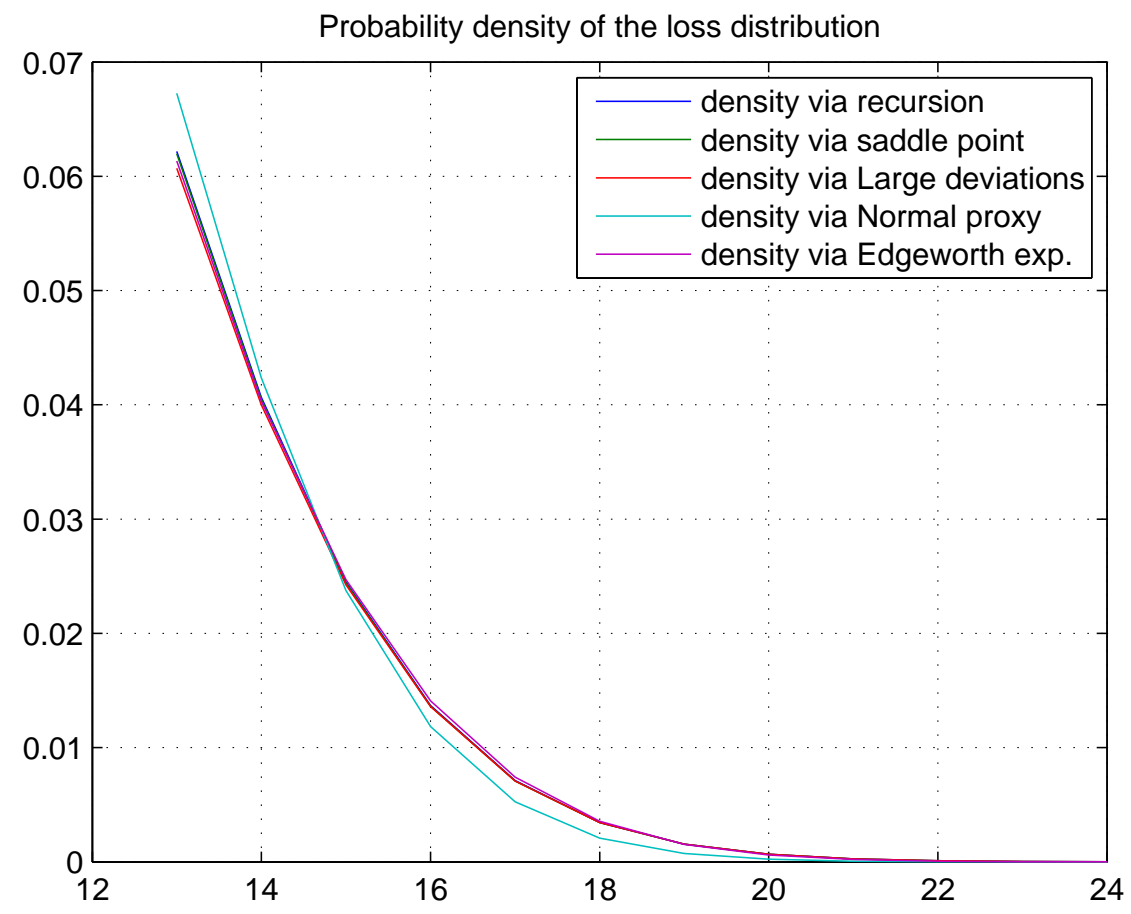

Now we compare the performance of each numerical method : the Saddle-point approximation (at order 2 and 4), the Large deviation approximation, the Normal proxy, the Edgeworth expansion (at order 3 and 4) with the recursion method, considered here as the benchmark numerical method. The portfolio considered is homogeneous: 
- Number of names $=100$;

- Recovery $=0 \%$;

- Individual spread $=50 \mathrm{bps}$, without term structure;

- Risk free rate $=0 \%$;

- Maturity of the Tranche swaps is 5Y, quarterly payments;

- Computed expected loss $=2,49 \%$

- Model: Gaussian copula with various flat correlations called "rho".

We consider 7 levels of correlation $\{2 \%, 10 \%, 20 \%, 30 \% ; 50 \% ; 60 \% ; 70 \%\}$ that largely includes the current levels of base correlations for the liquid credit indices (iTraxx, CDX etc.). The tranches considered span the entire capital structure from very thin equity to senior tranches.

We find the following tranche spreads: 


\begin{tabular}{|c|c|c|c|c|c|c|c|c|c|c|c|c|c|}
\hline tranche & $0 \%-2 \%$ & $0 \%-3 \%$ & $0 \%-4 \%$ & $2 \%-4 \%$ & $3 \%-6 \%$ & $4 \%-6 \%$ & $4 \%-8 \%$ & $6 \%-8 \%$ & $6 \%-9 \%$ & $8 \%-10 \%$ & $9 \%-12 \%$ & $10 \%-12 \%$ & $12 \%-14 \%$ \\
\hline Recursion & $2,994.0$ & $2,123.6$ & $1,596.7$ & 755.8 & 289.5 & 181.8 & 107.6 & 34.9 & 25.9 & 5.5 & 1.5 & 0.7 & 0.1 \\
\hline Saddle Point 2 & $2,915.4$ & $2,083.5$ & $1,578.8$ & 754.4 & 303.7 & 197.0 & 117.4 & 39.4 & 29.3 & 6.3 & 1.7 & 0.9 & 0.1 \\
\hline Saddle Point 4 & $2,945.5$ & $2,106.7$ & $1,589.9$ & 764.1 & 295.4 & 187.2 & 111.2 & 36.8 & 27.3 & 5.9 & 1.6 & 0.8 & 0.1 \\
\hline Large Dev & $3,620.2$ & $2,264.2$ & $1,630.6$ & 557.5 & 219.7 & 144.1 & 87.1 & 30.9 & 23.1 & 5.1 & 1.4 & 0.7 & 0.1 \\
\hline Normal & $2,934.7$ & $2,133.6$ & $1,611.8$ & 810.0 & 295.6 & 176.8 & 101.4 & 27.3 & 19.8 & 3.2 & 0.7 & 0.3 & 0.0 \\
\hline Jarrow-Rudd 3 & $2,912.7$ & $2,097.5$ & $1,587.3$ & 773.4 & 300.8 & 191.3 & 113.3 & 36.8 & 27.2 & 5.4 & 1.4 & 0.6 & 0.1 \\
\hline Jarrow-Rudd 4 & $2,833.5$ & $2,076.8$ & $1,579.1$ & 784.1 & 304.1 & 195.4 & 116.3 & 39.1 & 28.9 & 5.8 & 1.5 & 0.7 & 0.1 \\
\hline \multicolumn{14}{|l|}{ rho $=10 \%$} \\
\hline tranche & $0 \%-2 \%$ & $0 \%-3 \%$ & $0 \%-4 \%$ & $2 \%-4 \%$ & $3 \%-6 \%$ & $4 \%-6 \%$ & $4 \%-8 \%$ & $6 \%-8 \%$ & $6 \%-9 \%$ & $8 \%-10 \%$ & $9 \%-12 \%$ & $10 \%-12 \%$ & $12 \%-14 \%$ \\
\hline Recursion & $2,322.1$ & $1,733.6$ & $1,366.6$ & 718.0 & 367.8 & 282.1 & 198.9 & 118.2 & 99.2 & 51.1 & 28.6 & 22.6 & 10.1 \\
\hline Saddle Point 2 & $2,270.0$ & $1,713.0$ & $1,357.1$ & 727.6 & 374.6 & 288.0 & 203.2 & 121.0 & 101.6 & 52.5 & 29.4 & 23.2 & 10.4 \\
\hline Saddle Point 4 & $2,291.1$ & $1,722.6$ & $1,361.9$ & 725.6 & 371.8 & 285.3 & 201.2 & 119.5 & 100.3 & 51.7 & 28.9 & 22.8 & 10.3 \\
\hline Large Dev & $2,763.2$ & $1,869.2$ & $1,418.7$ & 585.3 & 309.2 & 240.7 & 171.3 & 103.7 & 87.2 & 45.4 & 25.6 & 20.4 & 9.3 \\
\hline Normal & $2,293.2$ & $1,733.8$ & $1,370.5$ & 741.0 & 372.7 & 283.6 & 198.8 & 116.5 & 97.5 & 49.7 & 27.6 & 21.7 & 9.7 \\
\hline Jarrow-Rudd 3 & $2,273.3$ & $1,717.8$ & $1,360.4$ & 732.4 & 374.4 & 287.0 & 202.2 & 119.9 & 100.6 & 51.7 & 28.9 & 22.8 & 10.2 \\
\hline Jarrow-Rudd 4 & $2,186.6$ & $1,694.1$ & $1,353.1$ & 759.9 & 385.5 & 294.5 & 206.6 & 121.5 & 101.7 & 51.8 & 28.8 & 22.6 & 10.1 \\
\hline \multicolumn{14}{|l|}{ rho $=20 \%$} \\
\hline tranche & $0 \%-2 \%$ & $0 \%-3 \%$ & $0 \%-4 \%$ & $2 \%-4 \%$ & $3 \%-6 \%$ & $4 \%-6 \%$ & $4 \%-8 \%$ & $6 \%-8 \%$ & $6 \%-9 \%$ & $8 \%-10 \%$ & $9 \%-12 \%$ & $10 \%-12 \%$ & $12 \%-14 \%$ \\
\hline Recursion & $1,774.2$ & $1,381.1$ & $1,128.5$ & 644.2 & 385.9 & 320.5 & 248.0 & 177.8 & 157.7 & 104.6 & 73.0 & 63.9 & 40.0 \\
\hline Saddle Point 2 & $1,742.1$ & $1,368.7$ & $1,122.5$ & 653.0 & 390.6 & 324.3 & 250.8 & 179.5 & 159.0 & 105.4 & 73.7 & 64.3 & 40.3 \\
\hline Saddle Point 4 & $1,754.8$ & $1,374.0$ & $1,125.2$ & 650.2 & 388.7 & 322.6 & 249.5 & 178.8 & 158.5 & 105.1 & 73.3 & 64.1 & 40.2 \\
\hline Large Dev & $2,090.1$ & $1,496.6$ & $1,182.5$ & 550.2 & 339.5 & 283.8 & 222.0 & 161.8 & 143.9 & 96.3 & 67.5 & 59.2 & 37.6 \\
\hline Normal & $1,757.8$ & $1,379.3$ & $1,129.2$ & 656.0 & 389.0 & 322.0 & 248.7 & 177.7 & 157.4 & 104.2 & 72.6 & 63.5 & 39.7 \\
\hline Jarrow-Rudd 3 & $1,745.3$ & $1,371.3$ & $1,124.2$ & 654.3 & 390.1 & 323.5 & 250.2 & 179.0 & 158.7 & 105.2 & 73.4 & 64.2 & 40.2 \\
\hline Jarrow-Rudd 4 & $1,679.6$ & $1,354.2$ & $1,118.8$ & 683.0 & 399.5 & 329.5 & 253.8 & 180.6 & 159.9 & 105.5 & 73.4 & 64.2 & 40.1 \\
\hline \multicolumn{14}{|l|}{ rho $=30 \%$} \\
\hline tranche & $0 \%-2 \%$ & $0 \%-3 \%$ & $0 \%-4 \%$ & $2 \%-4 \%$ & $3 \%-6 \%$ & $4 \%-6 \%$ & $4 \%-8 \%$ & $6 \%-8 \%$ & $6 \%-9 \%$ & $8 \%-10 \%$ & $9 \%-12 \%$ & $10 \%-12 \%$ & $12 \%-14 \%$ \\
\hline Recursion & $1,386.9$ & $1,113.5$ & 933.5 & 568.4 & 373.1 & 322.4 & 262.6 & 204.4 & 186.2 & 137.4 & 105.4 & 95.8 & 68.5 \\
\hline Saddle Point 2 & $1,366.3$ & $1,105.6$ & 929.4 & 575.1 & 376.2 & 325.0 & 264.4 & 205.4 & 187.3 & 138.4 & 105.7 & 96.0 & 68.8 \\
\hline Saddle Point 4 & $1,374.1$ & $1,108.7$ & 931.2 & 573.0 & 375.1 & 323.9 & 263.6 & 205.0 & 186.8 & 137.7 & 105.6 & 96.0 & 68.7 \\
\hline Large Dev & $1,615.6$ & $1,207.6$ & 981.8 & 501.3 & 337.3 & 293.1 & 240.9 & 189.9 & 173.2 & 129.2 & 99.3 & 89.8 & 65.7 \\
\hline Normal & $1,376.3$ & $1,111.5$ & 933.4 & 575.7 & 375.3 & 323.6 & 263.2 & 204.5 & 186.3 & 137.3 & 105.2 & 95.7 & 68.4 \\
\hline Jarrow-Rudd 3 & $1,368.4$ & $1,106.9$ & 930.5 & 575.7 & 376.1 & 324.5 & 264.1 & 205.3 & 187.0 & 137.8 & 105.7 & 96.1 & 68.7 \\
\hline Jarrow-Rudd 4 & $1,321.7$ & $1,094.8$ & 926.5 & 599.8 & 383.4 & 329.1 & 266.9 & 206.5 & 188.0 & 138.2 & 105.8 & 96.2 & 68.7 \\
\hline \multicolumn{14}{|l|}{ rho $=50 \%$} \\
\hline tranche & $0 \%-2 \%$ & $0 \%-3 \%$ & $0 \%-4 \%$ & $2 \%-4 \%$ & $3 \%-6 \%$ & $4 \%-6 \%$ & $4 \%-8 \%$ & $6 \%-8 \%$ & $6 \%-9 \%$ & $8 \%-10 \%$ & $9 \%-12 \%$ & $10 \%-12 \%$ & $12 \%-14 \%$ \\
\hline Recursion & 855.0 & 719.7 & 627.2 & 425.1 & 313.8 & 283.7 & 245.6 & 208.2 & 195.5 & 160.5 & 135.3 & 127.6 & 103.5 \\
\hline Saddle Point 2 & 845.9 & 715.5 & 625.6 & 429.4 & 315.8 & 284.3 & 246.3 & 209.1 & 195.8 & 160.6 & 136.1 & 128.3 & 103.0 \\
\hline Saddle Point 4 & 849.1 & 717.3 & 626.0 & 427.6 & 314.8 & 284.5 & 246.2 & 208.6 & 195.8 & 160.7 & 135.4 & 127.7 & 103.6 \\
\hline Large Dev & 982.3 & 780.2 & 661.8 & 388.3 & 291.9 & 265.2 & 232.2 & 199.7 & 185.9 & 151.2 & 130.6 & 123.8 & 99.3 \\
\hline Normal & 849.9 & 718.2 & 626.7 & 428.4 & 314.9 & 284.5 & 246.1 & 208.5 & 195.7 & 160.6 & 135.3 & 127.6 & 103.5 \\
\hline Jarrow-Rudd 3 & 846.7 & 716.5 & 625.6 & 428.9 & 315.3 & 284.8 & 246.4 & 208.7 & 195.9 & 160.8 & 135.5 & 127.7 & 103.6 \\
\hline Jarrow-Rudd 4 & 823.9 & 710.4 & 623.4 & 443.1 & 319.4 & 287.4 & 248.1 & 209.5 & 196.6 & 161.1 & 135.6 & 127.8 & 103.7 \\
\hline \multicolumn{14}{|l|}{ rho $=60 \%$} \\
\hline tranche & $0 \%-2 \%$ & $0 \%-3 \%$ & $0 \%-4 \%$ & $2 \%-4 \%$ & $3 \%-6 \%$ & $4 \%-6 \%$ & $4 \%-8 \%$ & $6 \%-8 \%$ & $6 \%-9 \%$ & $8 \%-10 \%$ & $9 \%-12 \%$ & $10 \%-12 \%$ & $12 \%-14 \%$ \\
\hline Recursion & 659.1 & 566.3 & 501.9 & 357.5 & 276.1 & 253.8 & 224.7 & 196.0 & 185.9 & 158.0 & 137.4 & 131.1 & 110.7 \\
\hline Saddle Point 2 & 652.1 & 564.1 & 500.4 & 360.9 & 277.2 & 255.2 & 225.3 & 195.9 & 185.7 & 158.2 & 138.2 & 131.9 & 110.3 \\
\hline Saddle Point 4 & 655.1 & 564.7 & 501.0 & 359.4 & 276.9 & 254.3 & 225.1 & 196.3 & 186.2 & 158.1 & 137.5 & 131.2 & 110.8 \\
\hline Large Dev & 755.4 & 613.4 & 530.7 & 330.4 & 259.1 & 237.4 & 213.0 & 189.0 & 178.0 & 150.4 & 133.5 & 127.9 & 108.3 \\
\hline Normal & 655.5 & 565.2 & 501.4 & 359.8 & 276.9 & 254.3 & 225.0 & 196.2 & 186.1 & 158.2 & 137.3 & 130.9 & 110.7 \\
\hline Jarrow-Rudd 3 & 653.5 & 564.1 & 500.7 & 360.2 & 277.1 & 254.5 & 225.3 & 196.5 & 186.3 & 158.1 & 137.5 & 131.2 & 110.8 \\
\hline Jarrow-Rudd 4 & 638.2 & 560.0 & 499.1 & 370.2 & 280.1 & 256.6 & 226.5 & 197.0 & 186.8 & 158.5 & 137.5 & 131.1 & 110.9 \\
\hline \multicolumn{14}{|l|}{ rho $=70 \%$} \\
\hline tranche & $0 \%-2 \%$ & $0 \%-3 \%$ & $0 \%-4 \%$ & $2 \%-4 \%$ & $3 \%-6 \%$ & $4 \%-6 \%$ & $4 \%-8 \%$ & $6 \%-8 \%$ & $6 \%-9 \%$ & $8 \%-10 \%$ & $9 \%-12 \%$ & $10 \%-12 \%$ & $12 \%-14 \%$ \\
\hline Recursion & 492.2 & 431.7 & 389.0 & 291.5 & 235.0 & 219.4 & 198.0 & 176.9 & 169.2 & 148.0 & 132.8 & 128.2 & 112.3 \\
\hline Saddle Point 2 & 488.8 & 429.7 & 387.8 & 292.3 & 236.7 & 221.3 & 198.4 & 175.7 & 168.7 & 148.9 & 133.5 & 128.8 & 112.1 \\
\hline Saddle Point 4 & 489.6 & 430.7 & 388.4 & 292.8 & 235.5 & 219.8 & 198.3 & 177.1 & 169.4 & 148.1 & 132.9 & 128.3 & 112.3 \\
\hline Large Dev & $1,018.2$ & 756.9 & 618.9 & 263.2 & 214.9 & 203.5 & 182.1 & 160.9 & 154.2 & 135.9 & 122.4 & 118.1 & 102.5 \\
\hline Normal & 489.7 & 430.8 & 388.7 & 293.1 & 235.4 & 219.4 & 198.4 & 177.6 & 169.8 & 148.1 & 132.4 & 127.7 & 112.4 \\
\hline Jarrow-Rudd 3 & 488.6 & 430.3 & 388.2 & 293.3 & 235.7 & 220.0 & 198.5 & 177.2 & 169.4 & 148.1 & 133.0 & 128.4 & 112.3 \\
\hline Jarrow-Rudd 4 & 479.5 & 427.2 & 387.4 & 299.8 & 237.8 & 220.4 & 199.3 & 178.3 & 170.2 & 148.0 & 132.6 & 128.0 & 112.6 \\
\hline
\end{tabular}

As we can see the tranches $[0 \%, 2 \%],[0 \%, 3 \%],[0 \%, 4 \%]$ and $[2 \%, 4 \%]$ have a spread that is monotonically decreasing function of correlation : those are the equity tranches for the basket considered while the next tranche $[3 \%, 6 \%]$ is the first mezzanine. The other tranches are senior mezzanine and senior tranches.

In the next table, we give the relative error, for each numerical method, between the spread and the

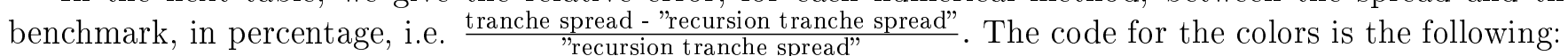

- green color: tranche spread relative error is smaller than $1 \%$

- blue color: tranche spread relative error is between $1 \%$ and $4 \%$

- red color: tranche spread relative error is greater than $20 \%$ 
We compute the Saddle-point at order 2 and 4, Edgeworth at order 3 and 4 and the Large deviation expansions.

\begin{tabular}{|c|c|c|c|c|c|c|c|c|c|c|c|c|c|}
\hline tranche & $0 \%-2 \%$ & $0 \%-3 \%$ & $0 \%-4 \%$ & $2 \%-4 \%$ & $3 \%-6 \%$ & $4 \%-6 \%$ & $4 \%-8 \%$ & $6 \%-8 \%$ & $6 \%-9 \%$ & $8 \%-10 \%$ & $9 \%-12 \%$ & $10 \%-12 \%$ & $12 \%-14 \%$ \\
\hline Saddle Point 2 & $2.6 \%$ & $1.9 \%$ & $1.1 \%$ & $0.2 \%$ & $4.9 \%$ & $8.3 \%$ & $9.0 \%$ & $13.0 \%$ & $13.2 \%$ & $14.6 \%$ & $15.0 \%$ & $15.2 \%$ & $15.4 \%$ \\
\hline Saddle Point 4 & $1.6 \%$ & $0.8 \%$ & $0.4 \%$ & $1.1 \%$ & $2.1 \%$ & $2.9 \%$ & $3.3 \%$ & $5.4 \%$ & $5.6 \%$ & $7.4 \%$ & $8.3 \%$ & $8.9 \%$ & $10.1 \%$ \\
\hline Large Dev & $20.9 \%$ & $6.6 \%$ & $2.1 \%$ & $26.2 \%$ & $24.1 \%$ & $20.7 \%$ & $19.1 \%$ & $11.4 \%$ & $11.0 \%$ & $6.5 \%$ & $4.9 \%$ & $4.0 \%$ & $2.7 \%$ \\
\hline Normal & $2.0 \%$ & $0.5 \%$ & $0.9 \%$ & $7.2 \%$ & $2.1 \%$ & $2.8 \%$ & $5.8 \%$ & $21.6 \%$ & $23.4 \%$ & $41.0 \%$ & $50.5 \%$ & $56.8 \%$ & $68.4 \%$ \\
\hline Jarrow-Rudd 3 & $2.7 \%$ & $1.2 \%$ & $0.6 \%$ & $2.3 \%$ & $3.9 \%$ & $5.2 \%$ & $5.2 \%$ & $5.6 \%$ & $5.1 \%$ & $1.0 \%$ & $7.2 \%$ & $12.4 \%$ & $25.3 \%$ \\
\hline Jarrow-Rudd 4 & $5.4 \%$ & $2.2 \%$ & $1.1 \%$ & $3.7 \%$ & $5.0 \%$ & $7.5 \%$ & $8.1 \%$ & $12.0 \%$ & $11.6 \%$ & $5.8 \%$ & $2.0 \%$ & $8.6 \%$ & $25.1 \%$ \\
\hline \multicolumn{14}{|l|}{ rho $=10 \%$} \\
\hline tranche & $0 \%-2 \%$ & $0 \%-3 \%$ & $0 \%-4 \%$ & $2 \%-4 \%$ & $3 \%-6 \%$ & $4 \%-6 \%$ & $4 \%-8 \%$ & $6 \%-8 \%$ & $6 \%-9 \%$ & $8 \%-10 \%$ & $9 \%-12 \%$ & $10 \%-12 \%$ & $12 \%-14 \%$ \\
\hline Saddle Point 2 & $2.2 \%$ & $1.2 \%$ & $0.7 \%$ & $1.3 \%$ & $1.9 \%$ & $2.1 \%$ & $2.2 \%$ & $2.4 \%$ & $2.5 \%$ & $2.7 \%$ & $2.6 \%$ & $2.7 \%$ & $2.7 \%$ \\
\hline Saddle Point 4 & $1.3 \%$ & $0.6 \%$ & $0.3 \%$ & $1.1 \%$ & $1.1 \%$ & $1.1 \%$ & $1.1 \%$ & $1.2 \%$ & $1.2 \%$ & $1.2 \%$ & $1.2 \%$ & $1.2 \%$ & $1.2 \%$ \\
\hline Large Dev & $19.0 \%$ & $7.8 \%$ & $3.8 \%$ & $18.5 \%$ & $15.9 \%$ & $14.7 \%$ & $13.9 \%$ & $12.3 \%$ & $12.0 \%$ & $11.2 \%$ & $10.4 \%$ & $9.4 \%$ & $8.4 \%$ \\
\hline Normal & $1.2 \%$ & $0.0 \%$ & $0.3 \%$ & $3.2 \%$ & $1.3 \%$ & $0.5 \%$ & $0.1 \%$ & $1.4 \%$ & $1.6 \%$ & $2.8 \%$ & $3.5 \%$ & $3.9 \%$ & $4.7 \%$ \\
\hline Jarrow-Rudd 3 & $2.1 \%$ & $0.9 \%$ & $0.5 \%$ & $2.0 \%$ & $1.8 \%$ & $1.7 \%$ & $1.6 \%$ & $1.5 \%$ & $1.4 \%$ & $1.3 \%$ & $1.1 \%$ & $1.1 \%$ & $0.9 \%$ \\
\hline Jarrow-Rudd 4 & $5.8 \%$ & $2.3 \%$ & $1.0 \%$ & $5.8 \%$ & $4.8 \%$ & $4.4 \%$ & $3.9 \%$ & $2.8 \%$ & $2.6 \%$ & $1.4 \%$ & $0.5 \%$ & $0.2 \%$ & $0.9 \%$ \\
\hline \multicolumn{14}{|l|}{ rho $=20 \%$} \\
\hline tranche & $0 \%-2 \%$ & $0 \%-3 \%$ & $0 \%-4 \%$ & $2 \%-4 \%$ & $3 \%-6 \%$ & $4 \%-6 \%$ & $4 \%-8 \%$ & $6 \%-8 \%$ & $6 \%-9 \%$ & $8 \%-10 \%$ & $9 \%-12 \%$ & $10 \%-12 \%$ & $12 \%-14 \%$ \\
\hline Saddle Point 2 & $1.8 \%$ & $0.9 \%$ & $0.5 \%$ & $1.4 \%$ & $1.2 \%$ & $1.2 \%$ & $1.1 \%$ & $1.0 \%$ & $0.9 \%$ & $0.8 \%$ & $0.9 \%$ & $0.7 \%$ & $0.8 \%$ \\
\hline Saddle Point 4 & $1.1 \%$ & $0.5 \%$ & $0.3 \%$ & $0.9 \%$ & $0.7 \%$ & $0.7 \%$ & $0.6 \%$ & $0.5 \%$ & $0.5 \%$ & $0.5 \%$ & $0.4 \%$ & $0.4 \%$ & $0.4 \%$ \\
\hline Large Dev & $17.8 \%$ & $8.4 \%$ & $4.8 \%$ & $14.6 \%$ & $12.0 \%$ & $11.5 \%$ & $10.5 \%$ & $9.0 \%$ & $8.7 \%$ & $8.0 \%$ & $7.6 \%$ & $7.2 \%$ & $5.9 \%$ \\
\hline Normal & $0.9 \%$ & $0.1 \%$ & $0.1 \%$ & $1.8 \%$ & $0.8 \%$ & $0.5 \%$ & $0.3 \%$ & $0.1 \%$ & $0.2 \%$ & $0.4 \%$ & $0.5 \%$ & $0.6 \%$ & $0.7 \%$ \\
\hline Jarrow-Rudd 3 & $1.6 \%$ & $0.7 \%$ & $0.4 \%$ & $1.6 \%$ & $1.1 \%$ & $1.0 \%$ & $0.9 \%$ & $0.7 \%$ & $0.7 \%$ & $0.6 \%$ & $0.5 \%$ & $0.5 \%$ & $0.4 \%$ \\
\hline Jarrow-Rudd 4 & $5.3 \%$ & $1.9 \%$ & $0.9 \%$ & $6.0 \%$ & $3.5 \%$ & $2.8 \%$ & $2.3 \%$ & $1.6 \%$ & $1.4 \%$ & $0.9 \%$ & $0.6 \%$ & $0.5 \%$ & $0.2 \%$ \\
\hline \multicolumn{14}{|l|}{ rho $=30 \%$} \\
\hline tranche & $0 \%-2 \%$ & $0 \%-3 \%$ & $0 \%-4 \%$ & $2 \%-4 \%$ & $3 \%-6 \%$ & $4 \%-6 \%$ & $4 \%-8 \%$ & $6 \%-8 \%$ & $6 \%-9 \%$ & $8 \%-10 \%$ & $9 \%-12 \%$ & $10 \%-12 \%$ & $12 \%-14 \%$ \\
\hline Saddle Point 2 & $1.5 \%$ & $0.7 \%$ & $0.4 \%$ & $1.2 \%$ & $0.8 \%$ & $0.8 \%$ & $0.7 \%$ & $0.5 \%$ & $0.6 \%$ & $0.7 \%$ & $0.4 \%$ & $0.3 \%$ & $0.3 \%$ \\
\hline Saddle Point 4 & $0.9 \%$ & $0.4 \%$ & $0.2 \%$ & $0.8 \%$ & $0.5 \%$ & $0.5 \%$ & $0.4 \%$ & $0.3 \%$ & $0.3 \%$ & $0.3 \%$ & $0.2 \%$ & $0.2 \%$ & $0.2 \%$ \\
\hline Large Dev & $16.5 \%$ & $8.4 \%$ & $5.2 \%$ & $11.8 \%$ & $9.6 \%$ & $9.1 \%$ & $8.3 \%$ & $7.1 \%$ & $7.0 \%$ & $6.0 \%$ & $5.7 \%$ & $6.2 \%$ & $4.2 \%$ \\
\hline Normal & $0.8 \%$ & $0.2 \%$ & $0.0 \%$ & $1.3 \%$ & $0.6 \%$ & $0.4 \%$ & $0.3 \%$ & $0.1 \%$ & $0.0 \%$ & $0.1 \%$ & $0.1 \%$ & $0.1 \%$ & $0.2 \%$ \\
\hline Jarrow-Rudd 3 & $1.3 \%$ & $0.6 \%$ & $0.3 \%$ & $1.3 \%$ & $0.8 \%$ & $0.7 \%$ & $0.6 \%$ & $0.4 \%$ & $0.4 \%$ & $0.3 \%$ & $0.3 \%$ & $0.3 \%$ & $0.2 \%$ \\
\hline Jarrow-Rudd 4 & $4.7 \%$ & $1.7 \%$ & $0.8 \%$ & $5.5 \%$ & $2.8 \%$ & $2.1 \%$ & $1.7 \%$ & $1.1 \%$ & $1.0 \%$ & $0.6 \%$ & $0.4 \%$ & $0.4 \%$ & $0.2 \%$ \\
\hline \multicolumn{14}{|l|}{ rho $=50 \%$} \\
\hline tranche & $0 \%-2 \%$ & $0 \%-3 \%$ & $0 \%-4 \%$ & $2 \%-4 \%$ & $3 \%-6 \%$ & $4 \%-6 \%$ & $4 \%-8 \%$ & $6 \%-8 \%$ & $6 \%-9 \%$ & $8 \%-10 \%$ & $9 \%-12 \%$ & $10 \%-12 \%$ & $12 \%-14 \%$ \\
\hline Saddle Point 2 & $1.1 \%$ & $0.6 \%$ & $0.3 \%$ & $1.0 \%$ & $0.6 \%$ & $0.2 \%$ & $0.3 \%$ & $0.4 \%$ & $0.2 \%$ & $0.1 \%$ & $0.6 \%$ & $0.6 \%$ & $0.5 \%$ \\
\hline Saddle Point 4 & $0.7 \%$ & $0.3 \%$ & $0.2 \%$ & $0.6 \%$ & $0.3 \%$ & $0.3 \%$ & $0.2 \%$ & $0.2 \%$ & $0.2 \%$ & $0.1 \%$ & $0.1 \%$ & $0.1 \%$ & $0.1 \%$ \\
\hline Large Dev & $14.9 \%$ & $8.4 \%$ & $5.5 \%$ & $8.7 \%$ & $7.0 \%$ & $6.5 \%$ & $5.5 \%$ & $4.1 \%$ & $4.9 \%$ & $5.8 \%$ & $3.5 \%$ & $2.9 \%$ & $4.1 \%$ \\
\hline Normal & $0.6 \%$ & $0.2 \%$ & $0.1 \%$ & $0.8 \%$ & $0.4 \%$ & $0.3 \%$ & $0.2 \%$ & $0.1 \%$ & $0.1 \%$ & $0.1 \%$ & $0.0 \%$ & $0.0 \%$ & $0.0 \%$ \\
\hline Jarrow-Rudd 3 & $1.0 \%$ & $0.4 \%$ & $0.3 \%$ & $0.9 \%$ & $0.5 \%$ & $0.4 \%$ & $0.3 \%$ & $0.2 \%$ & $0.2 \%$ & $0.2 \%$ & $0.1 \%$ & $0.1 \%$ & $0.1 \%$ \\
\hline Jarrow-Rudd 4 & $3.6 \%$ & $1.3 \%$ & $0.6 \%$ & $4.2 \%$ & $1.8 \%$ & $1.3 \%$ & $1.0 \%$ & $0.6 \%$ & $0.6 \%$ & $0.4 \%$ & $0.2 \%$ & $0.2 \%$ & $0.2 \%$ \\
\hline \multicolumn{14}{|l|}{ rho $=60 \%$} \\
\hline tranche & $0 \%-2 \%$ & $0 \%-3 \%$ & $0 \%-4 \%$ & $2 \%-4 \%$ & $3 \%-6 \%$ & $4 \%-6 \%$ & $4 \%-8 \%$ & $6 \%-8 \%$ & $6 \%-9 \%$ & $8 \%-10 \%$ & $9 \%-12 \%$ & $10 \%-12 \%$ & $12 \%-14 \%$ \\
\hline Saddle Point 2 & $1.1 \%$ & $0.4 \%$ & $0.3 \%$ & $0.9 \%$ & $0.4 \%$ & $0.6 \%$ & $0.3 \%$ & $0.1 \%$ & $0.1 \%$ & $0.2 \%$ & $0.6 \%$ & $0.6 \%$ & $0.3 \%$ \\
\hline Saddle Point 4 & $0.6 \%$ & $0.3 \%$ & $0.2 \%$ & $0.5 \%$ & $0.3 \%$ & $0.2 \%$ & $0.2 \%$ & $0.2 \%$ & $0.1 \%$ & $0.1 \%$ & $0.1 \%$ & $0.1 \%$ & $0.1 \%$ \\
\hline Large Dev & $14.6 \%$ & $8.3 \%$ & $5.8 \%$ & $7.6 \%$ & $6.1 \%$ & $6.5 \%$ & $5.2 \%$ & $3.6 \%$ & $4.2 \%$ & $4.8 \%$ & $2.8 \%$ & $2.4 \%$ & $2.2 \%$ \\
\hline Normal & $0.5 \%$ & $0.2 \%$ & $0.1 \%$ & $0.6 \%$ & $0.3 \%$ & $0.2 \%$ & $0.2 \%$ & $0.1 \%$ & $0.1 \%$ & $0.1 \%$ & $0.0 \%$ & $0.1 \%$ & $0.0 \%$ \\
\hline Jarrow-Rudd 3 & $0.8 \%$ & $0.4 \%$ & $0.2 \%$ & $0.7 \%$ & $0.4 \%$ & $0.3 \%$ & $0.3 \%$ & $0.2 \%$ & $0.2 \%$ & $0.1 \%$ & $0.1 \%$ & $0.1 \%$ & $0.1 \%$ \\
\hline Jarrow-Rudd 4 & $3.2 \%$ & $1.1 \%$ & $0.5 \%$ & $3.5 \%$ & $1.5 \%$ & $1.1 \%$ & $0.8 \%$ & $0.5 \%$ & $0.5 \%$ & $0.3 \%$ & $0.1 \%$ & $0.0 \%$ & $0.2 \%$ \\
\hline \multicolumn{14}{|l|}{ rho $=70 \%$} \\
\hline tranche & $0 \%-2 \%$ & $0 \%-3 \%$ & $0 \%-4 \%$ & $2 \%-4 \%$ & $3 \%-6 \%$ & $4 \%-6 \%$ & $4 \%-8 \%$ & $6 \%-8 \%$ & $6 \%-9 \%$ & $8 \%-10 \%$ & $9 \%-12 \%$ & $10 \%-12 \%$ & $12 \%-14 \%$ \\
\hline Saddle Point 2 & $0.7 \%$ & $0.5 \%$ & $0.3 \%$ & $0.3 \%$ & $0.7 \%$ & $0.9 \%$ & $0.2 \%$ & $0.7 \%$ & $0.3 \%$ & $0.6 \%$ & $0.5 \%$ & $0.5 \%$ & $0.2 \%$ \\
\hline Saddle Point 4 & $0.5 \%$ & $0.2 \%$ & $0.2 \%$ & $0.4 \%$ & $0.2 \%$ & $0.2 \%$ & $0.2 \%$ & $0.1 \%$ & $0.1 \%$ & $0.1 \%$ & $0.1 \%$ & $0.1 \%$ & $0.0 \%$ \\
\hline Large Dev & $106.9 \%$ & $75.3 \%$ & $59.1 \%$ & $9.7 \%$ & $8.6 \%$ & $7.2 \%$ & $8.1 \%$ & $9.1 \%$ & $8.9 \%$ & $8.2 \%$ & $7.9 \%$ & $7.9 \%$ & $8.6 \%$ \\
\hline Normal & $0.5 \%$ & $0.2 \%$ & $0.1 \%$ & $0.5 \%$ & $0.2 \%$ & $0.0 \%$ & $0.2 \%$ & $0.4 \%$ & $0.3 \%$ & $0.0 \%$ & $0.3 \%$ & $0.4 \%$ & $0.1 \%$ \\
\hline Jarrow-Rudd 3 & $0.7 \%$ & $0.3 \%$ & $0.2 \%$ & $0.6 \%$ & $0.3 \%$ & $0.3 \%$ & $0.2 \%$ & $0.1 \%$ & $0.1 \%$ & $0.0 \%$ & $0.1 \%$ & $0.1 \%$ & $0.1 \%$ \\
\hline Jarrow-Rudd 4 & $2.6 \%$ & $1.0 \%$ & $0.4 \%$ & $2.8 \%$ & $1.2 \%$ & $0.5 \%$ & $0.6 \%$ & $0.8 \%$ & $0.6 \%$ & $0.0 \%$ & $0.1 \%$ & $0.1 \%$ & $0.3 \%$ \\
\hline
\end{tabular}

We can see that equity tranches, i.e. "in the money" tranches relative to the current expected loss $(2.49 \%)$ are very well approximated with the normal proxy and whatever the correlation level. The Saddlepoint method is very robust, even for those equity tranches. But the large deviation approximation performs better for very senior tranches. On the other hand, it tends to give very bad results for equity tranches.

The most robust methods seems to be the Jarrow-Rudd approximation at order 4, except for very low correlations. 
Those results could be anticipated, given that the Saddle-point is a good approximation in the tail of the loss distribution, as well as the large deviation approximations. The observed robustness is more surprising for the equity tranches.

Other quantities are plotted in the last appendix: spread sensitivity (PV01), expected loss (tranche protection) and their relative errors with respect to the recursion.

\section{Conclusion}

In this paper, we compute higher order expansions for the Saddle-point and the Jarrow-Rudd methods applied to the loss distribution of a credit portfolio. We give the formula for the call on loss, which is necessary to feed the CDO tranches formula. We also propose an alternative numerical method based on large deviation approximations. In the light of the numerical results, we can say that the Saddle-point approximation and the Edgeworth approximation at order 4 are both robust, i.e. give good results whatever the seniority of the tranche. On the other hand the normal proxy should not be used to price senior tranches and the large deviations approximations should be used on the contrary only for the pricing of such tranches. Those results can be naturally applied to other "deterministic products" such as zero CDOs or CDO squares. The benefit of the Jarrow-Rudd approximation being its simplicity of implementation, its non dependance of the loss granularity and sign (short CDS could be considered here too and stochastic recoveries as well) and its non-dependency on a Saddle-point root to be found, makes it the fastest and most natural candidate to use for pricing, at least, vanilla index tranches. 


\section{Appendix}

\section{A Inversion formula}

We recall briefly the inversion of the Fourier Transform for $X=\sum_{i=1}^{n} X_{i}$ and $X_{i}$ are independent binomial distributions with $E\left(X_{i}\right)=p_{i}$

$$
M(\theta)=E\left[e^{\theta X}\right]=\sum_{k=0}^{n} \kappa_{k} \exp (\theta k)
$$

so for any $j \in\{0, \ldots, n\}$

$$
M\left(\frac{2 \pi i j}{n+1}\right)=\sum_{k=0}^{n} \kappa_{k} \exp \left(\frac{2 \pi i k j}{n+1}\right)
$$

as we have:

$$
\sum_{k=0}^{n} \exp \left(\frac{2 \pi i k j}{n+1}\right)=\frac{\exp (2 \pi i j)-1}{\exp \left(\frac{2 \pi i j}{n+1}\right)-1}=(n+1) \delta_{0}(j)=\left\{\begin{array}{c}
0 \text { if } j \neq 0 \\
n+1 \text { if } j=0
\end{array}\right.
$$

then we have the inversion formula:

$$
\kappa_{k}=\frac{1}{n+1} \sum_{j=0}^{n} M\left(\frac{2 \pi i j}{n+1}\right) \exp \left(-\frac{2 \pi i j k}{n+1}\right)
$$

Note that this is of the order $(n+1)^{2}$ in term of algorithmic complexity compared with $(n+1) \ln (n+1)$ if we use FFT. The only issue with FFT is that $n$ must be a power of 2 so we have to round it to the next power of 2 .

\section{B Useful integrals}

We use the same notations as in [3] for $J_{k}\left(m, \xi_{0}\right)=\frac{1}{2 \pi i} \int_{\xi_{0}-i \infty}^{\xi_{0}+i \infty} \frac{e^{\frac{1}{2} m\left(\xi-\xi_{0}\right)^{2}}}{\xi^{k}} d \xi$ :

$$
\left\{\begin{array}{c}
J_{0}\left(m, \xi_{0}\right)=\frac{1}{\sqrt{2 \pi m}} \\
J_{1}\left(m, \xi_{0}\right)=\operatorname{sign}\left(\xi_{0}\right) e^{\frac{1}{2} m \xi_{0}^{2}} \mathcal{N}\left(-\sqrt{m}\left|\xi_{0}\right|\right) \\
J_{2}\left(m, \xi_{0}\right)=\sqrt{\frac{m}{2 \pi}}-m\left|\xi_{0}\right| e^{\frac{1}{2} m \xi_{0}^{2}} \mathcal{N}\left(-\sqrt{m}\left|\xi_{0}\right|\right)
\end{array}\right.
$$

Note that by integration by parts we have:

$$
n J_{n+1}\left(m, \xi_{0}\right)=m\left(J_{n-1}\left(m, \xi_{0}\right)-\xi_{0} J_{n}\left(m, \xi_{0}\right)\right)
$$

We have by recursion for $I_{k}=\frac{1}{\sqrt{2 \pi}} \int_{-\infty}^{+\infty} x^{k} e^{-\frac{x^{2}}{2}} d x$ :

$$
\left\{\begin{array}{c}
I_{2 n}=\frac{1}{\sqrt{2 \pi}} \int_{-\infty}^{+\infty} x^{2 n} e^{-\frac{x^{2}}{2}} d x=\frac{(2 n-1) !}{2^{n-1}(n-1) !} \\
I_{2 n+1}=0
\end{array}\right.
$$


As a consequence:

$$
\int_{-\infty}^{+\infty} x^{2 n} e^{-\frac{x^{2}}{2} m} d x=\frac{\sqrt{2 \pi}}{m^{n+\frac{1}{2}}} \frac{(2 n-1) !}{2^{n-1}(n-1) !}
$$

and for any $\hat{\theta}$ and $c>0$ let define:

$$
c_{n}(m) \triangleq \frac{1}{2 i \pi} \int_{c-i \infty}^{c+i \infty}(\theta-\hat{\theta})^{n} e^{\frac{(\theta-\hat{\theta})^{2}}{2} m} d \theta
$$

We have $c_{2 n+1}(m)=0$ and:

$$
c_{2 n}(m)=\frac{1}{2 i \pi} \int_{\hat{\theta}-i \infty}^{\hat{\theta}+i \infty}(\theta-\hat{\theta})^{2 n} e^{\frac{(\theta-\hat{\theta})^{2}}{2} m} d \theta=\frac{(-1)^{n}}{\sqrt{2 \pi} m^{n+\frac{1}{2}}} \frac{(2 n-1) !}{2^{n-1}(n-1) !}
$$

More precisely:

- $2 n=0: c_{0}(m)=\frac{1}{2 i \pi} \int_{c-i \infty}^{c+i \infty} e^{\frac{(\theta-\hat{\theta})^{2}}{2} m} d \theta=\frac{1}{\sqrt{2 \pi m}}$

- $2 n=2: c_{2}(m)=\frac{1}{2 i \pi} \int_{c-i \infty}^{c+i \infty}(\theta-\hat{\theta})^{2} e^{\frac{(\theta-\hat{\theta})^{2}}{2} m} d \theta=-\frac{1}{\sqrt{2 \pi m} \cdot m}$

- $2 n=4: c_{4}(m)=\frac{1}{2 i \pi} \int_{c-i \infty}^{c+i \infty}(\theta-\hat{\theta})^{4} e^{\frac{(\theta-\hat{\theta})^{2}}{2} m} d \theta=\frac{3}{\sqrt{2 \pi m} \cdot m^{2}}$

- $2 n=6: c_{6}(m)=\frac{1}{2 i \pi} \int_{c-i \infty}^{c+i \infty}(\theta-\hat{\theta})^{6} e^{\frac{(\theta-\hat{\theta})^{2}}{2} m} d \theta=-\frac{15}{\sqrt{2 \pi m} \cdot m^{3}}$

- $2 n=8: c_{8}(m)=\frac{1}{2 i \pi} \int_{c-i \infty}^{c+i \infty}(\theta-\hat{\theta})^{8} e^{\frac{(\theta-\hat{\theta})^{2}}{2} m} d \theta=\frac{105}{\sqrt{2 \pi m} \cdot m^{4}}$

Let define:

$$
d_{n}(m) \triangleq \frac{1}{2 i \pi} \int_{\hat{\theta}-i \infty}^{\hat{\theta}+i \infty} \theta^{n} e^{\frac{(\theta-\hat{\theta})^{2}}{2} m} d \theta
$$

Unlike $c_{2 n+1}(m)$ the values of $d_{2 n+1}(m)$ are not trivial. We easily compute the first 8 terms:

- $d_{0}(m)=c_{0}(m)=\frac{1}{\sqrt{2 \pi m}}$

- $d_{1}(m)=c_{0}(m) \hat{\theta}=\frac{1}{\sqrt{2 \pi m}} \hat{\theta}$

- $d_{2}(m)=c_{2}(m)+c_{0}(m) \hat{\theta}^{2}=\frac{1}{\sqrt{2 \pi m}}\left(-\frac{1}{m}+\hat{\theta}^{2}\right)$

- $d_{3}(m)=3 c_{2}(m) \hat{\theta}+c_{0}(m) \hat{\theta}^{3}=\frac{1}{\sqrt{2 \pi m}}\left(-\frac{3}{m} \hat{\theta}+\hat{\theta}^{3}\right)$

- $d_{4}(m)=c_{4}(m)+6 c_{2}(m) \hat{\theta}^{2}+c_{0}(m) \hat{\theta}^{4}=\frac{1}{\sqrt{2 \pi m}}\left(\frac{3}{m^{2}}-\frac{6}{m} \hat{\theta}^{2}+\hat{\theta}^{4}\right)$

- $d_{5}(m)=5 c_{4}(m) \hat{\theta}+10 c_{2}(m) \hat{\theta}^{3}+c_{0}(m) \hat{\theta}^{5}=\frac{1}{\sqrt{2 \pi m}}\left(\frac{15}{m^{2}}-\frac{10}{m} \hat{\theta}^{3}+\hat{\theta}^{5}\right)$ 
- $d_{6}(m)=c_{6}(m)+15 c_{4}(m) \hat{\theta}^{2}+15 c_{2}(m) \hat{\theta}^{4}+c_{0}(m) \hat{\theta}^{6}=\frac{1}{\sqrt{2 \pi m}}\left(-\frac{15}{m^{3}}+\frac{45}{m^{2}} \hat{\theta}^{2}-\frac{15}{m} \hat{\theta}^{4}+\hat{\theta}^{6}\right)$

- $d_{7}(m)=7 c_{6}(m) \hat{\theta}+35 c_{4}(m) \hat{\theta}^{3}+21 c_{2}(m) \hat{\theta}^{5}+c_{0}(m) \hat{\theta}^{7}=\frac{1}{\sqrt{2 \pi m}}\left(-\frac{105}{m^{3}} \hat{\theta}+\frac{105}{m^{2}} \hat{\theta}^{3}-\frac{21}{m} \hat{\theta}^{5}+\hat{\theta}^{7}\right)$

- $d_{8}(m)=c_{8}(m)+28 c_{6}(m) \hat{\theta}^{2}+70 c_{4}(m) \hat{\theta}^{4}+28 c_{2}(m) \hat{\theta}^{6}+c_{0}(m) \hat{\theta}^{8}$ $=\frac{1}{\sqrt{2 \pi m}}\left(\frac{105}{m^{4}}-\frac{420}{m^{3}} \hat{\theta}^{2}+\frac{210}{m^{2}} \hat{\theta}^{4}-\frac{28}{m} \hat{\theta}^{6}+\hat{\theta}^{8}\right)$

Note finally that: $5 !=120 \quad ; \quad 6 !=720 \quad ; \quad 7 !=5040 \quad$ and $\quad 8 !=40320$.

\section{Computation of the cumulants derivatives}

\section{C.1 Cumulants of $X^{z}(t)$}

In the Large deviation approximation case, the sum in $k$ given by and are numerically intensive so we need to be able to compute $K_{t}^{z,(2)}(\hat{\theta}), K_{t}^{z,(3)}(\hat{\theta})$ and $K_{t}^{z,(4)}(\hat{\theta})$ very quickly. We define $q_{i}=1-p_{i}, \hat{p}_{i}=\frac{p_{i} e^{\hat{\theta}}}{q_{i}+p_{i} e^{\hat{\theta}}}$ and $\hat{q}_{i}=1-\hat{p}_{i}$. As a consequence we compute

$$
\begin{aligned}
& \frac{\partial \hat{p}_{i}}{\partial \theta}=\hat{p}_{i} \hat{q}_{i}=\hat{p}_{i}-\hat{p}_{i}^{2} \\
& \frac{\partial \hat{q}_{i}}{\partial \theta}=-\hat{p}_{i} \hat{q}_{i}=\hat{p}_{i}^{2}-\hat{p}_{i}
\end{aligned}
$$

and by derivation

$$
\frac{\partial \hat{p}_{i} \hat{q}_{i}}{\partial \theta}=\hat{p}_{i} \hat{q}_{i}\left(\hat{q}_{i}-\hat{p}_{i}\right)=2 \hat{p}_{i}^{3}-3 \hat{p}_{i}^{2}+\hat{p}_{i}
$$

by derivation again of the products

$$
\frac{\partial \hat{p}_{i} \hat{q}_{i}\left(\hat{q}_{i}-\hat{p}_{i}\right)}{\partial \theta}=\hat{p}_{i} \hat{q}_{i}\left(\hat{q}_{i}-\hat{p}_{i}\right)^{2}+\hat{p}_{i} \hat{q}_{i}\left(-\hat{p}_{i} \hat{q}_{i}-\hat{p}_{i} \hat{q}_{i}\right)
$$

noting that $\hat{q}_{i}=1-\hat{p}_{i}$ we have $\frac{\partial \hat{p}_{i} \hat{q}_{i}\left(\hat{q}_{i}-\hat{p}_{i}\right)}{\partial \theta}=\hat{p}_{i} \hat{q}_{i}\left(1-6 \hat{p}_{i}+6 \hat{p}_{i}^{2}\right)$ so finally

$$
\frac{\partial \hat{p}_{i} \hat{q}_{i}\left(\hat{q}_{i}-\hat{p}_{i}\right)}{\partial \theta}=\hat{p}_{i} \hat{q}_{i}\left(1-6 \hat{p}_{i} \hat{q}_{i}\right)
$$


we get

$$
\begin{aligned}
K_{t}^{z}(\hat{\theta}) & =\sum_{i=1}^{n} \ln \left(1-p_{i}+p_{i} e^{\hat{\theta}}\right) \\
K_{t}^{z,(1)}(\hat{\theta}) & =\sum_{i=1}^{n} \hat{p}_{i} \\
K_{t}^{z,(2)}(\hat{\theta}) & =\sum_{i=1}^{n} \hat{p}_{i}\left(1-\hat{p}_{i}\right)=\sum_{i=1}^{n}\left\{\hat{p}_{i}-\hat{p}_{i}^{2}\right\} \\
K_{t}^{z,(3)}(\hat{\theta}) & =\sum_{i=1}^{n} \hat{p}_{i} \hat{q}_{i}\left(1-2 \hat{p}_{i}\right)=\sum_{i=1}^{n}\left\{\hat{p}_{i}-3 \hat{p}_{i}^{2}+2 \hat{p}_{i}^{3}\right\} \\
K_{t}^{z,(4)}(\hat{\theta}) & =\sum_{i=1}^{n} \hat{p}_{i} \hat{q}_{i}\left(1-6 \hat{p}_{i} \hat{q}_{i}\right)=\sum_{i=1}^{n}\left\{\hat{p}_{i}-7 \hat{p}_{i}^{2}+12 \hat{p}_{i}^{3}-6 \hat{p}_{i}^{4}\right\}
\end{aligned}
$$

and

$$
\begin{aligned}
& K_{t}^{z,(5)}(\hat{\theta})=\sum_{i=1}^{n}\left\{\hat{p}_{i}-15 \cdot \hat{p}_{i}^{2}+50 \cdot \hat{p}_{i}^{3}-60 \cdot \hat{p}_{i}^{4}+24 \cdot \hat{p}_{i}^{5}\right\} \\
& K_{t}^{z,(6)}(\hat{\theta})=\sum_{i=1}^{n}\left\{\hat{p}_{i}-31 \cdot \hat{p}_{i}^{2}+180 \cdot \hat{p}_{i}^{3}-390 \cdot \hat{p}_{i}^{4}+360 \cdot \hat{p}_{i}^{5}-120 \cdot \hat{p}_{i}^{6}\right\} \\
& K_{t}^{z,(7)}(\hat{\theta})=\sum_{i=1}^{n}\left\{\hat{p}_{i}-63 \cdot \hat{p}_{i}^{2}+602 \cdot \hat{p}_{i}^{3}-2100 \cdot \hat{p}_{i}^{4}+3360 \cdot \hat{p}_{i}^{5}-2520 \cdot \hat{p}_{i}^{6}+720 \cdot \hat{p}_{i}^{7}\right\} \\
& K_{t}^{z,(8)}(\hat{\theta})=\sum_{i=1}^{n}\left\{\hat{p}_{i}-127 \cdot \hat{p}_{i}^{2}+1932 \cdot \hat{p}_{i}^{3}-10206 \cdot \hat{p}_{i}^{4}+25200 \cdot \hat{p}_{i}^{5}-31920 \cdot \hat{p}_{i}^{6}+20160 \cdot \hat{p}_{i}^{7}-5040 \cdot \hat{p}_{i}^{8}\right\}
\end{aligned}
$$

so we only need to generate vectors $\left(\hat{p}_{i}\right)_{i=1, n}$ and $\left(\hat{p}_{i} \hat{q}_{i}\right)_{i=1, n}$.

Note that

$$
\begin{aligned}
K_{t}^{z}(0) & =0 \\
K_{t}^{z,(1)}(0) & =\sum_{i=1}^{n} p_{i}=E^{Z}\left(X^{z}(t)\right)
\end{aligned}
$$

\section{C.2 Cumulants of $L^{z}(t)$}

Note that for the loss process $L^{z}(t)$ the formula are very similar:

$$
\hat{p}_{i}=\frac{p_{i} e^{a_{i} \hat{\theta}}}{q_{i}+p_{i} e^{a_{i} \hat{\theta}}}
$$


and

$$
\begin{aligned}
K_{t}^{z}(\hat{\theta}) & =\sum_{i=1}^{n} \ln \left(1-p_{i}+p_{i} e^{a_{i} \hat{\theta}}\right) \\
K_{t}^{z,(1)}(\hat{\theta}) & =\sum_{i=1}^{n} a_{i} \hat{p}_{i} \\
K_{t}^{z,(2)}(\hat{\theta}) & =\sum_{i=1}^{n} a_{i}^{2} \hat{p}_{i}\left(1-\hat{p}_{i}\right) \\
K_{t}^{z,(3)}(\hat{\theta}) & =\sum_{i=1}^{n} a_{i}^{3} \hat{p}_{i} \hat{q}_{i}\left(1-2 \hat{p}_{i}\right)
\end{aligned}
$$

\section{C.3 Relation between Cumulants and Moments}

For a given $\hat{\theta}$ let define the Esscher transform, i.e. the change of measure $X \longmapsto X \frac{e^{\hat{\theta} L}}{E\left(e^{\hat{\theta} L}\right)}$ as in 33 and $\hat{E}$ the associated expectation, i.e. $\hat{E}(X)=\frac{E\left(X e^{\hat{\theta} L}\right)}{E\left(e^{\hat{\theta} L}\right)}$. Then we can see that for $L^{z}(t)$ (and $X^{z}(t)$ ) we have:

$$
\begin{aligned}
K^{(1)}(\hat{\theta})= & \hat{E}(L) \\
K^{(2)}(\hat{\theta})= & \hat{\operatorname{Var}}(L)=\hat{E}\left(L^{2}\right)-\hat{E}(L)^{2} \\
= & \hat{E}\left((L-\hat{E}(L))^{2}\right) \\
K^{(3)}(\hat{\theta})= & \hat{E}\left(L^{3}\right)-\hat{E}(L) \hat{E}\left(L^{2}\right)-2 \hat{E}(L) \hat{\operatorname{Var}}(L) \\
= & \hat{E}\left(L^{3}\right)-3 \hat{E}(L) \hat{E}\left(L^{2}\right)+2 \hat{E}(L)^{3} \\
= & \hat{E}\left((L-\hat{E}(L))^{3}\right) \\
K^{(4)}(\hat{\theta})= & \hat{\operatorname{arr}}(L)\left(6 \hat{E}(L)^{2}-3 \hat{E}\left(L^{2}\right)\right) \\
& -3 \hat{E}(L)\left(\hat{E}\left(L^{3}\right)-\hat{E}(L) \hat{E}\left(L^{2}\right)\right) \\
& +\hat{E}\left(L^{4}\right)-\hat{E}(L) \hat{E}\left(L^{3}\right) \\
= & \hat{E}\left(L^{4}\right)-4 \hat{E}(L) \hat{E}\left(L^{3}\right)-3 \hat{E}\left(L^{2}\right)^{2}+12 \hat{E}\left(L^{2}\right) \hat{E}(L)^{2}-6 \hat{E}(L)^{4} \\
= & \hat{E}\left((L-\hat{E}(L))^{4}\right)-3 \hat{\operatorname{Var}}(L)^{2}
\end{aligned}
$$

So the relationship between the transformed cumulants $K^{(i)}(\hat{\theta})$ and transformed moments $\hat{E}\left(L^{i}\right)$ is independent of $\hat{\theta}$ : i.e. it is an invariant under the Esscher transform. 


\section{C.4 Moments of a Normal variable struck at $K$}

Let $\mu_{i}=E\left(X^{i}\right)=\int_{K}^{+\infty} x^{i} \phi(x) d x$ with $X$ a normal variable, centered with unit variance, $\phi$ given by (1) :

$$
\begin{aligned}
& \mu_{0}=\int_{K}^{+\infty} \phi(x) d x=\mathcal{N}(-K) \\
& \mu_{1}=\int_{K}^{+\infty} x \phi(x) d x=\phi(K) \\
& \mu_{2}=\int_{K}^{+\infty} x^{2} \phi(x) d x=K \phi(K)+\mathcal{N}(-K) \\
& \mu_{3}=\int_{K}^{+\infty} x^{3} \phi(x) d x=\left(K^{2}+2\right) \phi(K) \\
& \mu_{4}=\int_{K}^{+\infty} x^{4} \phi(x) d x=\left(K^{3}+3 K\right) \phi(K)+3 \mathcal{N}(-K) \\
& \mu_{5}=\int_{K}^{+\infty} x^{5} \phi(x) d x=\left(8+4 K^{2}+K^{4}\right) \phi(K)
\end{aligned}
$$

If the variable $X$ is $N\left(\mu, \sigma^{2}\right)$ let $\tilde{K}=\frac{K-\mu}{\sigma}$ :

$$
\begin{aligned}
\tilde{\mu}_{i} & =E\left(X^{i}\right)=\frac{1}{\sigma} \int_{K}^{+\infty} x^{i} 1 \phi\left(\frac{x-\mu}{\sigma}\right) d x \\
& =\int_{\tilde{K}}^{+\infty}(\sigma z+\mu)^{i} \phi(z) d z
\end{aligned}
$$

So

$$
\begin{aligned}
\tilde{\mu}_{0}= & \mathcal{N}(-\tilde{K}) \\
\tilde{\mu}_{1}= & \sigma \phi(\tilde{K})+\mu \mathcal{N}(-\tilde{K}) \\
\tilde{\mu}_{2}= & \left(2 \mu \sigma+\sigma^{2} \tilde{K}\right) \phi(\tilde{K})+\left(\mu^{2}+\sigma^{2}\right) \mathcal{N}(-\tilde{K}) \\
\tilde{\mu}_{3}= & \left(3 \mu^{2} \sigma+2 \sigma^{3}+3 \mu \sigma^{2} \tilde{K}+\sigma^{3} \tilde{K}^{2}\right) \phi(\tilde{K})+\left(3 \sigma^{2} \mu+\mu^{3}\right) \mathcal{N}(-\tilde{K}) \\
\tilde{\mu}_{4}= & \left(4 \mu^{3} \sigma+8 \mu \sigma^{3}+\left(3 \sigma^{4}+6 \sigma^{2} \mu^{2}\right) \tilde{K}+4 \sigma^{3} \mu \tilde{K}^{2}+\sigma^{4} \tilde{K}^{3}\right) \phi(\tilde{K}) \\
& +\left(3 \sigma^{4}+6 \sigma^{2} \mu^{2}+\mu^{4}\right) \mathcal{N}(-\tilde{K})
\end{aligned}
$$

\section{C.5 Cumulants of a Normal variable}

Let $X \sim N\left(\mu, \sigma^{2}\right)$ then we have an explicit formula for $K(\theta)$. It is actually a polynomial of degree 2 . So we already know that cumulants of higher orders ( larger than 3 ) are null :

$$
\begin{aligned}
K(\theta) & =\mu \theta+\frac{1}{2} \theta^{2} \sigma^{2} \\
K^{(1)}(\theta) & =\mu+\theta \sigma^{2} \\
K^{(2)}(\theta) & =\sigma^{2}=\operatorname{Var}(X) \\
K^{(i)}(\theta) & =0 \text { for } i \geq 3
\end{aligned}
$$




\section{Residue Theorem applied to the Saddle-point}

We recall here the Residue theorem. Given an analytic function $f(z)$, there is locally around $z_{0} \in \mathbb{C}$ a unique Laurent series given by $f(z)=\Sigma_{n \in \mathbb{Z}} a_{n}\left(z-z_{0}\right)^{n}$. If we integrate on a closed contour enclosing $z_{0}$, with interior $\Omega$, then

$$
\int_{\vec{\gamma}} f=\sum_{n=-\infty}^{-2} a_{n} \int_{\vec{\gamma}}\left(z-z_{0}\right)^{n}+a_{-1} \int_{\vec{\gamma}} \frac{1}{\left(z-z_{0}\right)}+\sum_{n=0}^{+\infty} a_{n} \int_{\vec{\gamma}}\left(z-z_{0}\right)^{n}
$$

The Cauchy integral theorem requires that the first and last terms vanish, so we have:

$$
\int_{\vec{\gamma}} f=a_{-1} \int_{\vec{\gamma}} \frac{1}{\left(z-z_{0}\right)}=2 i \pi a_{-1}
$$

If the contour $\vec{\gamma}$ encloses multiple poles, then the theorem gives the general result:

$$
\int_{\vec{\gamma}} f=2 i \pi \sum_{x \in \operatorname{Pôles}(\Omega)} \operatorname{Res}(f, x)
$$

$x$ is in Pôles $(\Omega)$ if $z \longmapsto(z-x)^{k} f(z)$ can be extended by continuity at $x$ for some $k \in \mathbb{N}$. The residue at $x$ for $f$ is noted $\operatorname{Res}(f, x)$ and is the coefficient $a_{-1}$ associated to the Laurent series of $f$ around $x$.

Example 1: if $f(z)=\frac{P(z)}{Q(z)}$ with $P(a)=Q(a)=0$ but $Q^{\prime}(a) \neq 0$ then $\operatorname{Res}(f, a)=\frac{P(a)}{Q^{\prime}(a)}$ otherwise we can do a limited development of $f$ around $a$.

Example 1: if $f(z)=\frac{a}{(z-1)^{2}}+\frac{b}{(z-1)}+\frac{c}{(z-2 i)}$ and $\vec{\gamma}$ enclosed $z=1$ and $z=2 i$ then

$$
\int_{\vec{\gamma}} f=2 i \pi(b+c)
$$

\section{E Loss Recursion}

We recall the general recursion described in [1], to compute both the number of defaults and the loss distribution recursively. The recursion technic described here is very powerful, as it gives the whole loss and number of defaults distribution. It is also very accurate and much faster than FFT. The formula described here are a bit different from those in Jacob's Risk paper.

Note also that the performance of the method in practice is very strongly dependant on the level of the implementation.

\section{E.1 Computation of the Number of defaults distribution}

Suppose that we have a basket of $n$ names and their default correlation in zero. Let $X_{T}=\sum_{i=1}^{n} 1_{\left\{\tau_{i}<T\right\}}$ for a fixed $T$. The survival probability of the $k^{\text {th }}$ to default, with $k \in\{1, \ldots, n\}$, is:

$$
Q_{0, T}^{k^{t h} T D}=Q\left(X_{T}=0\right)+Q\left(X_{T}=1\right)+\ldots+Q\left(X_{T}=k-1\right)
$$

We want to compute the number of defaults distribution for the portfolio, i.e. we want to compute accurately the probability $Q\left(X_{T}=k\right)$ for each $k \in\{0, \ldots, n\}$. The only quantities we know are the $q_{i}=$ 
$Q\left(\tau_{i}>T\right)$, i.e. the survival probability for each issuer $i$. Note that if $q_{i}=q$ for all $i$, then it is trivial, we have a multinomial distribution (mixture of independent iid binomial distributions) :

$$
Q\left(X_{T}=k\right)=C_{n}^{k} q^{n-k}(1-q)^{k}
$$

The idea in the general case where the $q_{i}$ are not the same, is to compute the $Q(k, l)$ recursively, where $Q(k, l)$ is the probability that the portfolio made of issuers $\{1, \ldots, k\}$ has exactly $l$ defaults $(0 \leq l \leq k)$.

Example :

- $k=0$ names in portfolio: $Q(k=0, l=0)=1$;

- $k=1$ names in portfolio:

$Q(k=1, l=0)=q_{1}$ no default from issuer 1 ;

$Q(k=1, l=1)=1-q_{1}$ one default from issuer 1 ;

- $k=2$ names in portfolio:

$Q(k=2, l=0)=q_{1} q_{2}$ no default from issuer 1 and 2 ;

$Q(k=2, l=1)=\left(1-q_{1}\right) q_{2}+\left(1-q_{2}\right) q_{1}$ one default from issuer 1 OR one default from issuer 2 ;

$Q(k=2, l=2)=\left(1-q_{1}\right)\left(1-q_{2}\right)$ one default from issuer 1 AND one default from issuer 2 ;

- ....and so on.

Now let make it more general : let suppose we already know $Q(k, l)$ for $l=0, \ldots, k$.

In order to compute $Q(k+1, l)$, from $Q(k, l-1)$ there are 2 possible outcomes:

either one name in the sub basket $\{1, \ldots, k\}$ defaults : so we have $l$ defaults with probability $Q(k, l)$;

or no name in the sub basket $\{1, \ldots, k\}$ defaults : so the defaults come from the new name added to the basket $\{k+1\}$ and its probability of defaulting is $\left(1-q_{k+1}\right)$.

Finally:

$$
\left\{\begin{array}{l}
Q(0,0)=1 \\
Q(k, 0)=q_{1} q_{2} \ldots q_{k} \text { for } k \in\{1, n\} \\
Q(k, k)=\left(1-q_{1}\right)\left(1-q_{2}\right) \ldots\left(1-q_{k}\right) \text { for } k \in\{1, n\}
\end{array}\right.
$$

and recursively for $l \in\{1, \ldots, k\}$ and $1<k<n$ :

$$
Q(k+1, l)=Q(k, l) \cdot q_{k+1}+Q(k, l-1) \cdot\left(1-q_{k+1}\right)
$$

\section{E.2 Computation of the Loss distribution}

Let suppose that each "ordered" name can lose $w_{i}$ for $i \in\{1, \ldots, n\}$ then the relation above is modified. $w_{i}$ must be an integer, i.e. a granularity adjustment should be done. It is also necessary to order the names in the following order : $w_{i} \leq w_{i+1}$. We also suppose $w_{1}>0$ otherwise this name can be removed from the basket (this can occur if the granularity is not small enough).

The loss accumulated a at time $T$ for the entire portfolio is:

$$
L_{T}=\sum_{i=1}^{n} w_{i} 1_{\left\{\tau_{i}<T\right\}}
$$


Let $Q(k, l)$ be the probability that the $\operatorname{loss} L_{T}^{k}=\sum_{i=1}^{k} w_{i} 1_{\left\{\tau_{i}<T\right\}}$ is exactly $l$ for a basket of $k$ names. Note that the expected loss is $E\left(L_{T}^{k}\right)=\sum_{i=1}^{k} w_{i}\left(1-q_{i}\right)$ and the "max loss" is $\operatorname{loss}_{k}=\sum_{i=1}^{k} w_{i}$. The general formula is:

$$
\left\{\begin{array}{l}
Q(0,0)=1 \\
Q(k, 0)=q_{1} q_{2} \ldots q_{K} \text { for } k \in\{1, \ldots, n\} \\
Q\left(k, l_{0 s s_{k}}\right)=\left(1-q_{1}\right)\left(1-q_{2}\right) \ldots\left(1-q_{k}\right) \text { for } k \in\{1, \ldots, n\}
\end{array}\right.
$$

We have a jump between $l=1$ and $l=w_{1}$ as the loss is either 0 or $w_{1}$ :

$$
\left\{\begin{array}{l}
Q(1, l)=0 \text { for } l \in\left\{1, \ldots w_{1}-1\right\} \\
Q\left(1, w_{1}\right)=1-q_{1}
\end{array}\right.
$$

Given that $Q(k, l)=0$ if $l<0$ and that the loss coming from name $(k+1)$ is $w_{k+1}$, we have by recursion for the $(k+1)$-names portfolio, for $l \in\left\{0, \ldots, \operatorname{loss}_{k}\right\}$ and $1 \leq k<n$ :

$$
Q(k+1, l) \equiv(k+1) \text { does not } \operatorname{default} \underbrace{Q(k, l) \cdot q_{k+1}}+(k+1) \operatorname{defaults} \underbrace{Q\left(k, l-w_{k+1}\right) \cdot\left(1-q_{k+1}\right)}
$$

in other words for $1 \leq k<n$ :

$\left\{\begin{array}{l}Q(k+1, l) \equiv(k+1) \text { does not default } \underbrace{Q(k, l) \cdot q_{k+1}} \text { for } l \in\left\{0, \ldots, w_{k+1}-1\right\} \\ Q\left(k+1, w_{k+1}\right) \equiv(k+1) \text { does not default } \underbrace{Q(k, l) \cdot q_{k+1}}+(k+1) \operatorname{defaults} \underbrace{q_{1} q_{2} \ldots q_{k}\left(1-q_{k+1}\right)} \\ Q(k+1, l) \equiv(k+1) \operatorname{does} \text { not default } \underbrace{Q(k, l) \cdot q_{k+1}}+Q, l-w_{k+1}) \cdot\left(1-q_{k+1}\right) \text { forl } l \in\left\{w_{k+1}+1, \ldots, l_{\text {loss }}\right\}\end{array}\right.$ and for $l \in\left\{l o s s_{k}+1, \ldots, \operatorname{loss}_{k+1}-1\right\}$ and $1 \leq k<n$ :

$$
Q(k+1, l)=0 .
$$

\section{F Higher order Saddle-point expansions}

By convention we write $K^{(i)}$ for $K_{t}^{z,(i)}(\hat{\theta})$ for any $i>0$, and we define $\Delta=(\theta-\hat{\theta})$

\section{F.1 Expansion for the density of $X^{z}(t) \sim 4^{t h}, 6^{t h}$ and $8^{t h}$ order expansion}

Let $\hat{\theta}$ be the Saddle-point. We develop $K_{t}^{z}(\theta)$ up to the order 8 around the Saddle-point $\hat{\theta}$. Using Appendix-B results we have:

$$
\begin{aligned}
& K_{t}^{z}(\theta)-\theta m_{0} \\
= & K_{t}^{z}(\hat{\theta})-\hat{\theta} m_{0}+\frac{\Delta^{2} K^{(2)}}{2}+\frac{\Delta^{3} K^{(3)}}{6}+\frac{\Delta^{4} K^{(4)}}{24} \\
& +\frac{\Delta^{5} K^{(5)}}{120}+\frac{\Delta^{6} K^{(6)}}{720}+\frac{\Delta^{7} K^{(7)}}{5040}+\frac{\Delta^{8} K^{(8)}}{40320}+o\left(\Delta^{8}\right)
\end{aligned}
$$


So using the fact the $e^{u}=1+u+\frac{u^{2}}{2}+\frac{u^{3}}{6}+o\left(u^{3}\right)$ :

$$
e^{K_{t}^{z}(\theta)-\theta m_{0}}=e^{K_{t}^{z}(\hat{\theta})-\hat{\theta} m_{0}} \times e^{\frac{1}{2} \Delta^{2} K^{(2)}} \times\left\{\begin{array}{c}
1+\frac{K^{(3)}}{6} \Delta^{3}+\frac{K^{(4)}}{24} \Delta^{4}+\frac{K^{(5)}}{120} \Delta^{5}+\left(\frac{K^{(6)}}{720}+\frac{K^{(3) 2}}{72}\right) \Delta^{6} \\
+\left(\frac{K^{(7)}}{5040}+\frac{K^{(3)} K^{(4)}}{144}\right) \Delta^{7}+\left(\frac{K^{(8)}}{40320}+\frac{K^{(4) 2}}{1152}+\frac{K^{(3)} K^{(5)}}{720}\right) \Delta^{8}+o\left(\Delta^{8}\right)
\end{array}\right\}
$$

In the expectation the odd terms vanish so we only consider coefficients of $\Delta^{2}, \Delta^{4}, \Delta^{6}$ and $\Delta^{8}$ :

$$
e^{K_{t}^{z}(\theta)-\theta m_{0}}=e^{K_{t}^{z}(\hat{\theta})-\hat{\theta} m_{0}} \times e^{\frac{1}{2} \Delta^{2} K^{(2)}} \times\left\{\begin{array}{c}
1+\frac{K^{(4)}}{24} \Delta^{4}+\left\{\frac{K^{(6)}}{720}+\frac{K^{(3) 2}}{72}\right\} \Delta^{6} \\
+\left\{\frac{K^{(8)}}{40320}+\frac{K^{(4) 2}}{1152}+\frac{K^{(3)} K^{(5)}}{720}\right\} \Delta^{8} \\
+\sum_{n<3} \Delta^{2 n+1} \alpha_{n}+o(\theta-\hat{\theta})^{8}
\end{array}\right\}
$$

Note that terms in $K^{(1)} K^{(7)}$ do not appear as $K^{(1)}$ is not in the sum from the beginning.

Integrating over $[c-i \infty ; c+i \infty]$ and using the definition of $c_{k}\left(K_{t}^{z,(2)}\right)$ in Appendix-B gives:

$$
\frac{1}{2 i \pi} \int_{c-i \infty}^{c+i \infty} e^{K_{t}^{z}(\theta)-\theta m_{0}} d \theta \simeq e^{K_{t}^{z}(\hat{\theta})-\hat{\theta} m_{0}} \times\left\{\begin{array}{c}
c_{0}\left(K^{(2)}\right)+\frac{K^{(4)}}{24} c_{4}\left(K^{(2)}\right)+\left(\frac{K^{(6)}}{720}+\frac{K^{(3) 2}}{72}\right) c_{6}\left(K^{(2)}\right) \\
+\left(\frac{K^{(8)}}{40320}+\frac{K^{(4) 2}}{1152}+\frac{K^{(3)} K^{(5)}}{720}\right) c_{8}\left(K^{(2)}\right)
\end{array}\right\}
$$

So for $n>1$, an $2 n^{t h}$ order expansion of $e^{K_{t}^{z}(\hat{\theta})-\hat{\theta} m_{0}}$ around $\hat{\theta}$ is equivalent to series in $\frac{1}{K^{(2)}}$ in power $\left(\frac{1}{K^{(2)}}\right)^{n}$ :

$$
Q^{t^{t h}}\left(X^{z}(t)=m_{0}\right) \simeq e^{K_{t}^{z}(\hat{\theta})-\hat{\theta} m_{0}} \times \frac{1}{\sqrt{2 \pi K^{(2)}}} \times\left\{\begin{array}{c}
1+\frac{K^{(4)}}{8 K^{(2) 2}}-\left\{\frac{K^{(6)}}{48}+\frac{5 K^{(3) 2}}{24}\right\} \frac{1}{K^{(2) 3}} \\
+\left\{\frac{K^{(8)}}{384}+\frac{35 K^{(4) 2}}{384}+\frac{7 K^{(3)} K^{(5)}}{48}\right\} \frac{1}{K^{(2) 4}}
\end{array}\right\}
$$

\section{F.2 Expansion for the tail of $Q\left(X^{z}(t) \geq m_{0}\right) \sim 4^{t h}$ and $6^{t h}$ order expansion}

As for the quadratic approximation, we have to take into account the fact that $\hat{\theta}$ may be positive or negative.

When it is positive, then:

$$
\frac{1}{2 i \pi} \int_{c-i \infty}^{c+i \infty} \frac{e^{K_{t}^{z}(\theta)-\theta m_{0}}}{\theta} d \theta=\frac{1}{2 i \pi} \int_{\hat{\theta}-i \infty}^{\hat{\theta}+i \infty} \frac{e^{K_{t}^{z}(\theta)-\theta m_{0}}}{\theta} d \theta
$$

otherwise:

$$
\frac{1}{2 i \pi} \int_{c-i \infty}^{c+i \infty} \frac{e^{K_{t}^{z}(\theta)-\theta m_{0}}}{\theta} d \theta=1+\frac{1}{2 i \pi} \int_{\hat{\theta}-i \infty}^{\hat{\theta}+i \infty} \frac{e^{K_{t}^{z}(\theta)-\theta m_{0}}}{\theta} d \theta
$$

Let suppose $\hat{\theta}>0$ then expanding $K_{t}^{z}(\theta)-\theta m_{0}$ around $\hat{\theta}$ to order 6 as is and using $e^{u}=1+u+\frac{u^{2}}{2}+o\left(u^{2}\right)$ we find:

$$
\begin{aligned}
& e^{K_{t}^{z}(\theta)-\theta m_{0}}=e^{K_{t}^{z}(\hat{\theta})-\hat{\theta} m_{0}} \times e^{\frac{1}{2} \Delta^{2} K^{(2)}} \times \\
& \left\{1+\frac{K^{(3)}}{6} \Delta^{3}+\frac{K^{(4)}}{24} \Delta^{4}+\frac{K^{(5)}}{120} \Delta^{5}+\left(\frac{K^{(6)}}{720}+\frac{K^{(3) 2}}{72}\right) \Delta^{6}+o\left(\Delta^{6}\right)\right\}
\end{aligned}
$$


Now we expand $\Delta^{k}=(\theta-\hat{\theta})^{k}$ and factorize in $\theta$ :

$$
e^{K_{t}^{z}(\theta)-\theta m_{0}}=e^{K_{t}^{z}(\hat{\theta})-\hat{\theta} m_{0}} \times e^{\frac{1}{2} \Delta^{2} K^{(2)}} \times\left(\sum_{k=0}^{6} \alpha_{k} \theta^{k}+o\left(\Delta^{6}\right)\right)
$$

with $\alpha_{k}$ and $K^{(k)}=K_{t}^{z,(k)}(\hat{\theta})$ are functions of $\hat{\theta}$ only $(\operatorname{not} \theta)$ :

- $\alpha_{0}=1-\frac{1}{6} K^{(3)} \hat{\theta}^{3}+\frac{1}{24} K^{(4)} \hat{\theta}^{4}-\frac{1}{120} K^{(5)} \hat{\theta}^{5}+\frac{1}{720} K^{(6)} \hat{\theta}^{6}+\frac{1}{72} K^{(3) 2} \hat{\theta}^{6}$

- $\alpha_{1}=\frac{1}{2} K^{(3)} \hat{\theta}^{2}-\frac{1}{6} K^{(4)} \hat{\theta}^{3}+\frac{1}{24} K^{(5)} \hat{\theta}^{4}-\frac{1}{120} K^{(6)} \hat{\theta}^{5}-\frac{1}{12} K^{(3) 2} \hat{\theta}^{5}$

- $\alpha_{2}=-\frac{1}{2} K^{(3)} \hat{\theta}+\frac{1}{4} K^{(4)} \hat{\theta}^{2}-\frac{1}{12} K^{(5)} \hat{\theta}^{3}+\frac{1}{48} K^{(6)} \hat{\theta}^{4}+\frac{5}{24} K^{(3) 2} \hat{\theta}^{4}$

- $\alpha_{3}=\frac{1}{6} K^{(3)}-\frac{1}{6} K^{(4)} \hat{\theta}+\frac{1}{12} K^{(5)} \hat{\theta}^{2}-\frac{1}{36} K^{(6)} \hat{\theta}^{3}-\frac{5}{18} K^{(3) 2} \hat{\theta}^{3}$

- $\alpha_{4}=\frac{1}{24} K^{(4)}-\frac{1}{24} K^{(5)} \hat{\theta}+\frac{1}{48} K^{(6)} \hat{\theta}^{2}+\frac{5}{24} K^{(3) 2} \hat{\theta}^{2}$

- $\alpha_{5}=\frac{1}{120} K^{(5)}-\frac{1}{120} K^{(6)} \hat{\theta}-\frac{1}{12} K^{(3) 2} \hat{\theta}$

- $\alpha_{6}=\frac{1}{720} K^{(6)}+\frac{1}{72} K^{(3) 2}$

Then dividing by $\theta$ and Integrating on ] $-i \infty,+i \infty[$ gives

$$
\begin{aligned}
& \frac{1}{2 i \pi} \int_{c-i \infty}^{c+i \infty} \frac{e^{K_{t}^{z}(\theta)-\theta m_{0}}}{\theta} d \theta \\
\simeq & e^{K_{t}^{z}(\hat{\theta})-\hat{\theta} m_{0}} \times\left\{\alpha_{0} J_{1}\left(K^{(2)}, \hat{\theta}\right)+\alpha_{1} J_{0}\left(K^{(2)}, \hat{\theta}\right)+\alpha_{2} d_{1}\left(K^{(2)}\right)+\alpha_{3} d_{2}\left(K^{(2)}\right) \ldots+\alpha_{6} d_{5}\left(K^{(2)}\right)\right\}
\end{aligned}
$$

where $J_{k}(.,$.$) and d_{k}($.$) are given in Appendix-B.$

A simplification and factorization finally gives for $\hat{\theta}>0$ :

$$
\begin{aligned}
& Q\left(X^{z}(t) \geq m_{0}\right)=\frac{1}{2 i \pi} \int_{c-i \infty}^{c+i \infty} \frac{e^{K_{t}^{z}(\theta)-\theta m_{0}}}{\theta} d \theta \\
& \simeq e^{K_{t}^{z}(\hat{\theta})-\hat{\theta} m_{0}} e^{\frac{1}{2} K^{(2)} \hat{\theta}^{2}} \mathcal{N}\left(-\sqrt{K^{(2)}} \hat{\theta}\right) \times\left\{1-\frac{K^{(3)} \hat{\theta}^{3}}{6}+\frac{K^{(4)} \hat{\theta}^{4}}{24}-\frac{K^{(5)} \hat{\theta}^{5}}{120}+\left(\frac{K^{(6)}}{720}+\frac{K^{(3) 2}}{72}\right) \hat{\theta}^{6}\right\} \\
&+\frac{e^{K_{t}^{z}(\hat{\theta})-\hat{\theta} m_{0}}}{72 \sqrt{2 \pi}\left(K^{(2)}\right)^{\frac{5}{2}}} \times\left\{\begin{array}{c}
3 K^{(2)}\left(1-\hat{\theta}^{2} K^{(2)}\right)\left(\hat{\theta} K^{(4)}-4 K^{(3)}+\frac{\hat{\theta}^{2}}{5}\left(\frac{\hat{\theta} K^{(6)}}{6}-K^{(5)}\right)\right) \\
-\hat{\theta} K^{(3) 2} \cdot\left(18-\hat{\theta}^{2} K^{(2)}+\hat{\theta}^{4} K^{(2) 2}\right) \\
+\frac{9 K^{(5)}}{5}+K^{(6)}\left(\frac{3}{2}-\frac{9 \hat{\theta}}{5}\right)+15 K^{(3) 2}
\end{array}\right\}
\end{aligned}
$$


The general formula for $\hat{\theta} \in \mathbb{R}$ is:

$$
\begin{aligned}
& Q^{6^{t h}}\left(X^{z}(t) \geq m_{0}\right) \\
\simeq & 1_{\{\hat{\theta} \leq 0\}}+\operatorname{sign}(\hat{\theta}) e^{K_{t}^{z}(\hat{\theta})-\hat{\theta} m_{0}} \times e^{\frac{1}{2} K^{(2)} \hat{\theta}^{2}} \mathcal{N}\left(-\sqrt{K^{(2)}}|\hat{\theta}|\right) \times \\
& \left\{1-\frac{K^{(3)} \hat{\theta}^{3}}{6}+\frac{K^{(4)} \hat{\theta}^{4}}{24}-\frac{K^{(5)} \hat{\theta}^{5}}{120}+\left(\frac{K^{(6)}}{720}+\frac{K^{(3) 2}}{72}\right) \hat{\theta}^{6}\right\} \\
& +\frac{e^{K_{t}^{z}(\hat{\theta})-\hat{\theta} m_{0}}}{72 \sqrt{2 \pi}\left(K^{(2)}\right)^{\frac{5}{2}}} \times\left\{\begin{array}{c}
3 K^{(2)}\left(1-\hat{\theta}^{2} K^{(2)}\right)\left[\hat{\theta} K^{(4)}-4 K^{(3)}+\frac{\hat{\theta}^{2}}{5}\left(\frac{\hat{\theta} K^{(6)}}{6}-K^{(5)}\right)\right] \\
-\hat{\theta} K^{(3) 2} \cdot\left(18-\hat{\theta}^{2} K^{(2)}+\hat{\theta}^{4} K^{(2) 2}\right) \\
+\frac{9 K^{(5)}}{5}+K^{(6)}\left(\frac{3}{2}-\frac{9 \hat{\theta}}{5}\right)+15 K^{(3) 2}
\end{array}\right\}
\end{aligned}
$$

Note that a $4^{\text {th }}$ order expansion is given by the following result:

$$
e^{K_{t}^{z}(\theta)-\theta m_{0}}=e^{K_{t}^{z}(\hat{\theta})-\hat{\theta} m_{0}} \times e^{\frac{1}{2} \Delta^{2} K^{(2)}} \times\left(\sum_{k=0}^{4} \beta_{k} \theta^{k}+o\left(\Delta^{4}\right)\right)
$$

with:

- $\beta_{0}=1-\frac{1}{6} K_{3} \theta_{0}^{3}+\frac{1}{24} K_{4} \theta_{0}^{4}$

- $\beta_{1}=\frac{1}{2} K_{3} \theta_{0}^{2}-\frac{1}{6} K_{4} \theta_{0}^{3}$

- $\beta_{2}=-\frac{1}{2} K_{3} \theta_{0}+\frac{1}{4} K_{4} \theta_{0}^{2}$

- $\beta_{3}=\frac{1}{6} K_{3}-\frac{1}{6} K_{4} \theta_{0}$

- $\beta_{4}=\frac{1}{24} K_{4}$

so:

$$
\begin{aligned}
& \frac{1}{2 i \pi} \int_{c-i \infty}^{c+i \infty} \frac{e^{K_{t}^{z}(\theta)-\theta m_{0}}}{\theta} d \theta \\
\simeq & e^{K_{t}^{z}(\hat{\theta})-\hat{\theta} m_{0}} \times\left\{\beta_{0} J_{1}\left(K^{(2)}, \hat{\theta}\right)+\beta_{1} J_{0}\left(K^{(2)}, \hat{\theta}\right)+\beta_{2} d_{1}\left(K^{(2)}\right)+\beta_{3} d_{2}\left(K^{(2)}\right)+\beta_{4} d_{3}\left(K^{(2)}\right)\right\}
\end{aligned}
$$

Then

$$
\begin{aligned}
& Q^{4^{t h}}\left(X^{z}(t) \geq m_{0}\right) \\
& \simeq 1_{\{\hat{\theta} \leq 0\}} \quad+\operatorname{sign}(\hat{\theta}) e^{K_{t}^{z}(\hat{\theta})-\hat{\theta} m_{0}} e^{\frac{1}{2} K^{(2)} \hat{\theta}^{2}} \mathcal{N}\left(-\sqrt{K^{(2)}}|\hat{\theta}|\right)\left(1-\frac{K^{(3)} \hat{\theta}^{3}}{6}+\frac{K^{(4)} \hat{\theta}^{4}}{24}\right) \\
&+\frac{e^{K_{t}^{z}(\hat{\theta})-\hat{\theta} m_{0}}}{24 \sqrt{2 \pi}\left(K^{(2)}\right)^{\frac{3}{2}}}\left(1-\hat{\theta}^{2} K^{(2)}\right)\left(\hat{\theta} K^{(4)}-4 K^{(3)}\right)
\end{aligned}
$$


F.3 Expansion for the call on Loss $E\left(L^{z}(t)-l_{0}\right)_{+} \sim 4^{\text {th }}$ and $6^{\text {th }}$ order expansion The call on loss for $\theta>0$ is given by :

$$
E\left(L^{z}(t)-l_{0}\right)_{+}=\frac{1}{2 i \pi} \int_{c-i \infty}^{c+i \infty} \frac{e^{K_{t}^{z}(\theta)-\theta l_{0}}}{\theta^{2}} d \theta
$$

and more generally:

$$
E\left(L^{z}(t)-l_{0}\right)_{+} \simeq 1_{\{\hat{\theta} \leq 0\}} \cdot\left(E^{Z}\left(L^{z}(t)\right)-l_{0}\right)+e^{K_{t}^{z}(\hat{\theta})-\hat{\theta} l_{0}} \times S^{k t h}
$$

with $S^{k t h}$ given below.

Using again we have

$$
\frac{1}{2 i \pi} \int_{c-i \infty}^{c+i \infty} \frac{e^{K_{t}^{z}(\theta)-\theta l_{0}}}{\theta^{2}} d \theta \simeq e^{K_{t}^{z}(\hat{\theta})-\hat{\theta} l_{0}} S^{6 t h}
$$

We compute the sum $S^{6 t h}$ :

$$
\begin{aligned}
S^{6 t h}= & \alpha_{0} J_{2}\left(K^{(2)}, \hat{\theta}\right)+\alpha_{1} J_{1}\left(K^{(2)}, \hat{\theta}\right)+\alpha_{2} J_{0}\left(K^{(2)}, \hat{\theta}\right)+\alpha_{3} d_{1}\left(K^{(2)}\right)+\alpha_{4} d_{2}\left(K^{(2)}\right) \\
& +\alpha_{5} d_{3}\left(K^{(2)}\right)+\alpha_{6} d_{4}\left(K^{(2)}\right)
\end{aligned}
$$

more precisely:

$$
\begin{aligned}
S^{6 t h}= & \hat{\theta}^{2} \operatorname{sign}(\hat{\theta}) \mathcal{N}\left(-\sqrt{K^{(2)}}|\hat{\theta}|\right) e^{\frac{1}{2} K^{(2)} \hat{\theta}^{2}}\left\{\frac{K^{(3)}}{2}-\frac{K^{(4)} \hat{\theta}}{6}+\frac{K^{(5)} \hat{\theta}^{2}}{24}-\frac{K^{(6)} \hat{\theta}^{3}}{120}-\frac{K^{(3) 2} \hat{\theta}^{3}}{12}\right\} \\
& -|\hat{\theta}| K^{(2)} \mathcal{N}\left(-\sqrt{K^{(2)}}|\hat{\theta}|\right) e^{\frac{1}{2} K^{(2)} \hat{\theta}^{2}}\left\{1-\frac{K^{(3)} \hat{\theta}^{3}}{6}+\frac{K^{(4)} \hat{\theta}^{4}}{24}-\frac{K^{(5)} \hat{\theta}^{5}}{120}+\frac{K^{(6)} \hat{\theta}^{6}}{720}+\frac{K^{(3) 2} \hat{\theta}^{6}}{72}\right\} \\
& +\frac{1}{\sqrt{2 \pi} K^{(2) \frac{5}{2}}}\left\{\begin{array}{c}
K^{(2) 2} \hat{\theta}\left(-\frac{K^{(3)}}{3}+\frac{K^{(4)} \hat{\theta}}{8}-\frac{K^{(5)} \hat{\theta}^{2}}{30}+\frac{K^{(6)} \hat{\theta}^{3}}{144}+\frac{5 K^{(3) 2} \hat{\theta}^{3}}{72}\right) \\
+K^{(2)}\left(-\frac{K^{(4)}}{24}+\frac{K^{(5)} \hat{\theta}}{60}-\frac{K^{(6)} \hat{\theta}^{2}}{240}-\frac{K^{(3) 2} \hat{\theta}^{2}}{24}\right) \\
+K^{(2) 3}\left(1-\frac{K^{(3)} \hat{\theta}^{3}}{6}+\frac{K^{(4)} \hat{\theta}^{4}}{24}-\frac{K^{(5)} \hat{\theta}^{5}}{120}+\frac{K^{(6)} \hat{\theta}^{6}}{720}+\frac{K^{(3) 2} \hat{\theta}^{6}}{72}\right) \\
+\frac{K^{(6)}}{240}+\frac{K^{(3) 2}}{24}
\end{array}\right\}
\end{aligned}
$$

A development at order 4 leads to:

$$
\frac{1}{2 i \pi} \int_{c-i \infty}^{c+i \infty} \frac{e^{K_{t}^{z}(\theta)-\theta l_{0}}}{\theta^{2}} d \theta \simeq e^{K_{t}^{z}(\hat{\theta})-\hat{\theta} l_{0}} S^{4 t h}
$$

with:

$$
S^{4 t h}=\beta_{0} J_{2}\left(K^{(2)}, \hat{\theta}\right)+\beta_{1} J_{1}\left(K^{(2)}, \hat{\theta}\right)+\beta_{2} J_{0}\left(K^{(2)}, \hat{\theta}\right)+\beta_{3} d_{1}\left(K^{(2)}\right)+\beta_{4} d_{2}\left(K^{(2)}\right)
$$


more precisely:

$$
\begin{aligned}
S^{4 t h}= & \hat{\theta}^{2} \operatorname{sign}(\hat{\theta}) \mathcal{N}\left(-\sqrt{K^{(2)}}|\hat{\theta}|\right) e^{\frac{1}{2} K^{(2)} \hat{\theta}^{2}}\left\{\frac{K^{(3)}}{2}-\frac{K^{(4)} \hat{\theta}}{6}\right\} \\
& -|\hat{\theta}| K^{(2)} \mathcal{N}\left(-\sqrt{K^{(2)}}|\hat{\theta}|\right) e^{\frac{1}{2} K^{(2)} \hat{\theta}^{2}}\left\{1-\frac{K^{(3)} \hat{\theta}^{3}}{6}+\frac{K^{(4)} \hat{\theta}^{4}}{24}\right\} \\
& +\frac{1}{\sqrt{2 \pi} K^{(2) \frac{3}{2}}}\left\{K^{(2) 2}-\frac{K^{(4)}}{24}+K^{(2)} \hat{\theta}\left(-\frac{K^{(3)}}{3}+\frac{K^{(4)} \hat{\theta}}{8}-\frac{K^{(2)} K^{(3)} \hat{\theta}^{2}}{6}+\frac{K^{(2)} K^{(4)} \hat{\theta}^{3}}{24}\right)\right\}
\end{aligned}
$$

\section{G Large Deviation Approximations}

We extend the proof in [9] by computing higher order terms in the Taylor expansions.

The idea is to find, for a given $m_{0}$ and a given positive $k$, a relation between $q_{k}=Q\left(X^{z}(t)=m_{0}+k\right)$ and $q_{0}=Q\left(X^{z}(t)=m_{0}\right)$. For that we are going to exploit the properties of the Saddle-point at $m_{0}+k$. More precisely let define $\hat{\theta}$ and $\hat{\theta}_{k}$ the solutions of :

$$
\begin{aligned}
K^{(1)}(\hat{\theta}) & =m_{0} \\
K^{(1)}\left(\hat{\theta}_{k}\right) & =m_{0}+k
\end{aligned}
$$

and

$$
\Delta_{k}=\hat{\theta}_{k}-\hat{\theta}
$$

For sake of clarity let define :

$$
K^{(j)}(\hat{\theta}):=K_{j}
$$

Basically, we are going to express $\Delta_{k}$ as a function of the cumulants of $X^{z}(t)$ at point $m_{0}$. In [9] we already assume that we have an approximation of $q_{k}$ given by Daniel's formula. Consequently:

$$
q_{k}=\frac{e^{K\left(\hat{\theta}_{k}\right)-\left(m_{0}+k\right) \hat{\theta}_{k}}}{\sqrt{2 \pi K^{(2)}\left(\hat{\theta}_{k}\right)}}\left\{1+\frac{K^{(4)}\left(\hat{\theta}_{k}\right)}{8 K^{(2)}\left(\hat{\theta}_{k}\right)^{2}}-\frac{5 K^{(3)}\left(\hat{\theta}_{k}\right)^{2}}{24 K^{(2)}\left(\hat{\theta}_{k}\right)^{3}}\right\}
$$

so

$$
\begin{aligned}
\ln \frac{q_{k}}{q_{0}}= & K\left(\hat{\theta}_{k}\right)-K(\hat{\theta})-\left\{\left(m_{0}+k\right) \hat{\theta}_{k}-m_{0} \hat{\theta}\right\} \\
& -\frac{1}{2}\left\{\ln K^{(2)}\left(\hat{\theta}_{k}\right)-\ln K^{(2)}(\hat{\theta})\right\} \\
+ & \ln \left\{1+\frac{K^{(4)}\left(\hat{\theta}_{k}\right)}{8 K^{(2)}\left(\hat{\theta}_{k}\right)^{2}}-\frac{5 K^{(3)}\left(\hat{\theta}_{k}\right)^{2}}{24 K^{(2)}\left(\hat{\theta}_{k}\right)^{3}}\right\} \\
& -\ln \left\{1+\frac{K^{(4)}(\hat{\theta})}{8 K^{(2)}(\hat{\theta})^{2}}-\frac{5 K^{(3)}(\hat{\theta})^{2}}{24 K^{(2)}(\hat{\theta})^{3}}\right\}
\end{aligned}
$$


Now we have to express everything in term of $k$ and $\hat{\theta}$. The Taylor expansions in $\Delta_{k}$ are stopped after $k=3$ as we will see, even order $k=2$ is accurate enough.

Computation of $\Delta_{k}:$ Using 23 we get

$$
K^{(1)}\left(\hat{\theta}_{k}\right)-K^{(1)}(\hat{\theta})=k
$$

and with a Taylor expansion of $K^{(1)}\left(\hat{\theta}_{k}\right)$ around $\hat{\theta}$ up to order 3 ,we get

$$
K^{(1)}\left(\hat{\theta}_{k}\right)-K^{(1)}(\hat{\theta}) \approx \Delta_{k} K_{2}+\frac{\Delta_{k}^{2}}{2} K_{3}+\frac{\Delta_{k}^{3}}{6} K_{4}
$$

So

$$
\Delta_{k} K_{2}+\frac{\Delta_{k}^{2}}{2} K_{3}+\frac{\Delta_{k}^{3}}{6} K_{4} \approx k
$$

and

$$
\Delta_{k} \approx \frac{k}{K_{2}}-\frac{K_{3}}{2 K_{2}} \Delta_{k}^{2}-\frac{K_{4}}{6 K_{2}} \Delta_{k}^{3}
$$

and $\Delta_{k}$ can be expressed recursively as a function of $k, k^{2} \ldots$ by re-injection $\frac{k}{K_{2}}$ in $\Delta_{k}^{2}$ and $\Delta_{k}^{3}$ the previous equation:

$$
\Delta_{k} \approx \frac{1}{K_{2}} k-\frac{K_{3}^{2}}{2 K_{2}^{3}} k^{2}+\left(\frac{K_{3}^{2}}{2 K_{2}^{5}}-\frac{K_{4}}{6 K_{2}^{4}}\right) k^{3}
$$

we now have a relationship between the Saddle-point $\hat{\theta}_{k}, \hat{\theta}$ and the cumulants $\left(K_{j}\right)_{j=2,4}$. Note that we could easily go further in the development but as we can see numerically order 3 is sufficient.

Computation of $\left(m_{0}+k\right) \hat{\theta}_{k}-m_{0} \hat{\theta}:$ We have $\hat{\theta}_{k}=\hat{\theta}+\Delta_{k}$ so

$$
\left(m_{0}+k\right) \hat{\theta}_{k}-m_{0} \hat{\theta}=\left(m_{0}+k\right) \Delta_{k}+k \hat{\theta}
$$

Computation of $K\left(\hat{\theta}_{k}\right)-K(\hat{\theta})$ : We compute $K\left(\hat{\theta}_{k}\right)-K(\hat{\theta})$ using a Taylor expansion at order 3 in $\Delta_{k}:$

$$
\begin{aligned}
K\left(\hat{\theta}_{k}\right)-K(\hat{\theta}) & \approx \Delta_{k} K_{1}+\frac{1}{2} \Delta_{k}^{2} K_{2}+\frac{1}{6} \Delta_{k}^{3} K_{3} \\
& \approx \Delta_{k} m_{0}+\frac{1}{2} \Delta_{k}^{2} K_{2}+\frac{1}{6} \Delta_{k}^{3} K_{3}
\end{aligned}
$$

Computation of $\ln K^{(2)}\left(\hat{\theta}_{k}\right)-\ln K^{(2)}(\hat{\theta}) \quad$ We have again by developing around $\hat{\theta}$ :

$$
\begin{aligned}
\ln K^{(2)}\left(\hat{\theta}_{k}\right)-\ln K^{(2)}(\hat{\theta}) \approx & \frac{K_{3}}{K_{2}} \Delta_{k}+\frac{1}{2}\left(\frac{K_{4}}{K_{2}}-\left(\frac{K_{3}}{K_{2}}\right)^{2}\right) \Delta_{k}^{2} \\
& +\frac{1}{6}\left(\frac{K_{5}}{K_{2}}-3 \frac{K_{4} K_{3}}{K_{2}^{2}}+2 \frac{K_{3}^{3}}{K_{2}^{3}}\right) \Delta_{k}^{3}
\end{aligned}
$$


Computation of $\ln \left\{1+\frac{K^{(4)}\left(\hat{\theta}_{k}\right)}{8 K^{(2)}\left(\hat{\theta}_{k}\right)^{2}}-\frac{5 K^{(3)}\left(\hat{\theta}_{k}\right)^{2}}{24 K^{(2)}\left(\hat{\theta}_{k}\right)^{3}}\right\} \quad$ Note that $g(\hat{\theta})=\frac{K^{(4)}(\hat{\theta})}{8 K^{(2)}(\hat{\theta})^{2}}-\frac{5 K^{(3)}(\hat{\theta})^{2}}{24 K^{(2)}(\hat{\theta})^{3}}$ is already the residue of an expansion so is very small. We can write

$$
\begin{aligned}
\ln \left\{1+g\left(\hat{\theta}_{k}\right)\right\}-\ln \{1+g(\hat{\theta})\} & \approx \frac{g^{\prime}(\hat{\theta})}{1+g(\hat{\theta})} \Delta_{k} \\
& \approx g^{\prime}(\hat{\theta})(1-g(\hat{\theta})) \Delta_{k}+\frac{1}{2} g^{\prime \prime}(\hat{\theta}) \Delta_{k}^{2}
\end{aligned}
$$

with

$$
\begin{aligned}
g(\hat{\theta}) & =\frac{K_{4}}{8 K_{2}^{2}}-\frac{5 K_{3}^{2}}{24 K_{2}^{3}} \\
g^{\prime}(\hat{\theta}) & =\frac{K_{5}}{8 K_{2}^{2}}-\frac{2 K_{3} K_{4}}{3 K_{2}^{3}}+\frac{5 K_{3}^{3}}{8 K_{2}^{4}} \\
g^{\prime \prime}(\hat{\theta}) & =\frac{K_{6}}{8 K_{2}^{2}}-\frac{2 K_{4}^{2}}{3 K_{2}^{3}}-\frac{11 K_{3} K_{5}}{12 K_{2}^{3}}+\frac{31 K_{3}^{2} K_{4}}{8 K_{2}^{4}}-\frac{5 K_{4}^{4}}{2 K_{2}^{5}}
\end{aligned}
$$

Computation of $\ln \frac{q_{k}}{q_{0}}$ :power in $\frac{1}{K_{2}^{j}}$ up to $j=2$ only Using the approximation (27) we have $\Delta_{k} \approx \frac{k}{K_{2}}$. Replacing $\Delta_{k}$ in the formulas (28) (29) (30) we (32) finally have if we retain only terms in $\frac{1}{K_{2}}$ and $\frac{1}{K_{2}^{j}}$ :

$$
\ln \frac{q_{k}}{q_{0}} \approx-k \hat{\theta}-\frac{1}{2 K_{2}} k^{2}-\frac{K_{3}}{2 K_{2}} k
$$

so the relation between the density $Q\left(X^{z}(t)=m_{0}+k\right)$ and $Q\left(X^{z}(t)=m_{0}\right)$ is finally:

$$
Q\left(X^{z}(t)=m_{0}+k\right)=Q\left(X^{z}(t)=m_{0}\right) \exp \left(-k\left(\hat{\theta}+\frac{K_{3}}{2 K_{2}}\right)-\frac{1}{2 K_{2}} k^{2}\right)
$$

\section{G.1 Higher order expansions:}

Order 2: The previous result consist in expanding the polynomial in $k^{2}$ but to use $\Delta_{k} \approx \frac{k}{K_{2}}$. We can refine the result with higher order terms in $\frac{1}{K_{2}^{j}}$ by replacing $\Delta_{k}$ with (27) in (28) (29) (30). We finally find:

$$
Q\left(X^{z}(t)=m_{0}+k\right)=Q\left(X^{z}(t)=m_{0}\right) \exp \left(a_{1} k+a_{2} k^{2}\right)
$$

with

$$
\begin{aligned}
& a_{1}=-\theta-\frac{1}{2} \frac{K_{3}}{K_{2}^{2}}+\frac{1}{8} \frac{K_{5}}{K_{2}^{3}}-\frac{2}{3} \frac{K_{3} K_{4}}{K_{2}^{4}}+\frac{5}{8} \frac{K_{3}^{3}}{K_{2}^{5}} \\
& a_{2}=-\frac{1}{2} \frac{1}{K_{2}}-\frac{1}{4} \frac{K_{4}}{K_{2}^{3}}+\left(\frac{1}{4} K_{3}^{2}+\frac{1}{4} K_{3}^{3}\right) \frac{1}{K_{2}^{4}}-\frac{5}{16} \frac{K_{3}^{5}}{K_{2}^{7}}-\frac{1}{16} \frac{K_{3}^{2} K_{5}}{K_{2}^{5}}+\frac{1}{3} \frac{K_{3}^{3} K_{4}}{K_{2}^{6}}
\end{aligned}
$$


Order 3: If we go up to order $k^{3}$, we have to rewrite (27):

$$
\Delta_{k}=\frac{1}{K_{2}} k-\frac{\left(K_{3}\right)^{2}}{2\left(K_{2}\right)^{3}} k^{2}+\left(\frac{\left(K_{3}\right)^{2}}{2\left(K_{2}\right)^{5}}-\frac{K_{4}}{6 K_{2}^{4}}\right) k^{3}
$$

and also (32) :

$$
\ln \left\{1+g\left(\hat{\theta}_{k}\right)\right\}-\ln \{1+g(\hat{\theta})\} \approx g_{1} \Delta+\frac{1}{2} g_{2} \Delta^{2}
$$

with

$$
\begin{aligned}
g_{1} & =\frac{K_{5}}{8 K_{2}^{2}}-\frac{2 K_{3} K_{4}}{3 K_{2}^{3}}+\frac{5 K_{3}^{3}}{8 K_{2}^{4}} \\
g_{2} & =\frac{K_{6}}{8 K_{2}^{2}}-\frac{2 K_{4}^{2}}{3 K_{2}^{3}}-\frac{11 K_{3} K_{5}}{12 K_{2}^{3}}+\frac{31 K_{3}^{2} K_{4}}{8 K_{2}^{4}}-\frac{5 K_{4}^{4}}{2 K_{2}^{5}}
\end{aligned}
$$

and (30) :

$$
\ln K^{(2)}\left(\hat{\theta}_{k}\right)-\ln K^{(2)}(\hat{\theta}) \approx-\frac{1}{2}\left(\frac{K_{3}}{K_{2}} \Delta+\frac{1}{2}\left(\frac{K_{4}}{K_{2}}-\frac{\left(K_{3}\right)^{2}}{\left(K_{2}\right)^{2}}\right) \Delta^{2}+\frac{1}{6}\left(\frac{K_{5}}{K_{2}}-3 \frac{K_{4} K_{3}}{K_{2}^{2}}+2 \frac{K_{3}^{3}}{K_{2}^{3}}\right) \Delta^{3}\right)
$$

We then find by expanding in $k$ :

$$
Q\left(X^{z}(t)=m_{0}+k\right)=Q\left(X^{z}(t)=m_{0}\right) \exp \left(b_{1} k+b_{2} k^{2}+b_{3} k^{3}\right)
$$

with

$$
\begin{aligned}
b_{1}=-\theta-\frac{1}{2} \frac{K_{3}}{K_{2}^{2}}+\frac{1}{8} \frac{K_{5}}{K_{2}^{3}}-\frac{2}{3} \frac{K_{3} K_{4}}{K_{2}^{4}}+\frac{5}{8} \frac{K_{3}^{3}}{K_{2}^{5}} \\
b_{2}=-\frac{1}{2} \frac{1}{K_{2}}-\frac{1}{4} \frac{K_{4}}{K_{2}^{3}}+\left(\frac{1}{16} K_{6}+\frac{1}{4} K_{3}^{2}+\frac{1}{4} K_{3}^{3}\right) \frac{1}{K_{2}^{4}}-\left(\frac{11}{24} K_{3} K_{5}+\frac{1}{3} K_{4}^{2}+\frac{1}{16} K_{3}^{2} K_{5}\right) \frac{1}{K_{2}^{5}} \\
+\left(\frac{31}{16} K_{3}^{2} K_{4}+\frac{1}{3} K_{3}^{3} K_{4}\right) \frac{1}{K_{2}^{6}}-\left(\frac{5}{16} K_{3}^{5}+\frac{5}{4} K_{4}^{4}\right) \frac{1}{K_{2}^{7}} \\
b_{3}=\frac{1}{6} \frac{K_{3}}{K_{2}^{3}}-\frac{1}{12} \frac{K_{5}}{K_{2}^{4}}+\left(\frac{1}{3} K_{3} K_{4}+\frac{1}{4} K_{3}^{2} K_{4}\right) \frac{1}{K_{2}^{5}}-\left(\frac{1}{48} K_{4}+\frac{5}{12} K_{3}^{3}+\frac{1}{4} K_{3}^{4}+\frac{1}{16} K_{3}^{2} K_{6}\right) \frac{1}{K_{2}^{6}} \\
+\left(\frac{1}{9} K_{3} K_{4}^{2}+\frac{1}{16} K_{3}^{2} K_{5}+\frac{11}{24} K_{3}^{3} K_{5}+\frac{1}{3} K_{3}^{2} K_{4}^{2}\right) \frac{1}{K_{2}^{7}}-\left(\frac{7}{16} K_{3}^{3} K_{4}+\frac{31}{16} K_{3}^{4} K_{4}\right) \frac{1}{K_{2}^{8}} \\
+\left(\frac{5}{16} K_{3}^{5}+\frac{5}{4} K_{3}^{2} K_{4}^{4}\right) \frac{1}{K_{2}^{9}}
\end{aligned}
$$




\section{H Additional numerical results}

The spreads differences reported in the part Numerical results are based on a portfolio of 100 names with identical recovery $(=0)$ and identical spread $(=50 \mathrm{bps})$. The tranches maturity is $5 \mathrm{Y}$ and with assume zero discounting rate. The tranches expected loss computed for those tranches is given by the following table:

\begin{tabular}{|c|c|c|c|c|c|c|c|c|c|c|c|c|c|}
\hline tranche & $0 \%-2 \%$ & $0 \%-3 \%$ & $0 \%-4 \%$ & $2 \%-4 \%$ & $3 \%-6 \%$ & $4 \%-6 \%$ & $4 \%-8 \%$ & $6 \%-8 \%$ & $6 \%-9 \%$ & $8 \%-10 \%$ & $9 \%-12 \%$ & $10 \%-12 \%$ & $12 \%-14 \%$ \\
\hline Recursion & $73.5 \%$ & $62.2 \%$ & $52.2 \%$ & $30.8 \%$ & $12.9 \%$ & $8.2 \%$ & $4.9 \%$ & $1.6 \%$ & $1.2 \%$ & $0.3 \%$ & $0.1 \%$ & $0.0 \%$ & $0.0 \%$ \\
\hline Saddle Point 2 & $72.9 \%$ & $61.4 \%$ & $51.7 \%$ & $30.6 \%$ & $13.5 \%$ & $8.9 \%$ & $5.4 \%$ & $1.8 \%$ & $1.4 \%$ & $0.3 \%$ & $0.1 \%$ & $0.0 \%$ & $0.0 \%$ \\
\hline Saddle Point 4 & $73.0 \%$ & $61.9 \%$ & $52.0 \%$ & $31.1 \%$ & $13.1 \%$ & $8.5 \%$ & $5.1 \%$ & $1.7 \%$ & $1.3 \%$ & $0.3 \%$ & $0.1 \%$ & $0.0 \%$ & $0.0 \%$ \\
\hline Large Dev & $82.6 \%$ & $65.3 \%$ & $53.1 \%$ & $23.6 \%$ & $9.9 \%$ & $6.6 \%$ & $4.0 \%$ & $1.4 \%$ & $1.1 \%$ & $0.2 \%$ & $0.1 \%$ & $0.0 \%$ & $0.0 \%$ \\
\hline Normal & $72.2 \%$ & $62.2 \%$ & $52.5 \%$ & $32.9 \%$ & $13.2 \%$ & $8.0 \%$ & $4.6 \%$ & $1.3 \%$ & $0.9 \%$ & $0.1 \%$ & $0.0 \%$ & $0.0 \%$ & $0.0 \%$ \\
\hline Jarrow-Rudd 3 & $72.5 \%$ & $61.7 \%$ & $51.9 \%$ & $31.3 \%$ & $13.3 \%$ & $8.7 \%$ & $5.2 \%$ & $1.7 \%$ & $1.3 \%$ & $0.3 \%$ & $0.1 \%$ & $0.0 \%$ & $0.0 \%$ \\
\hline Jarrow-Rudd 4 & $72.1 \%$ & $61.5 \%$ & $51.8 \%$ & $31.5 \%$ & $13.4 \%$ & $8.8 \%$ & $5.3 \%$ & $1.8 \%$ & $1.3 \%$ & $0.3 \%$ & $0.1 \%$ & $0.0 \%$ & $0.0 \%$ \\
\hline \multicolumn{14}{|l|}{ rho $=10 \%$} \\
\hline tranche & $0 \%-2 \%$ & $0 \%-3 \%$ & $0 \%-4 \%$ & $2 \%-4 \%$ & $3 \%-6 \%$ & $4 \%-6 \%$ & $4 \%-8 \%$ & $6 \%-8 \%$ & $6 \%-9 \%$ & $8 \%-10 \%$ & $9 \%-12 \%$ & $10 \%-12 \%$ & $12 \%-14 \%$ \\
\hline Recursion & $63.5 \%$ & $53.9 \%$ & $46.2 \%$ & $28.9 \%$ & $16.0 \%$ & $12.5 \%$ & $8.9 \%$ & $5.4 \%$ & $4.5 \%$ & $2.4 \%$ & $1.3 \%$ & $1.0 \%$ & $0.5 \%$ \\
\hline Saddle Point 2 & $62.7 \%$ & $53.5 \%$ & $45.9 \%$ & $29.2 \%$ & $16.2 \%$ & $12.7 \%$ & $9.1 \%$ & $5.5 \%$ & $4.6 \%$ & $2.4 \%$ & $1.4 \%$ & $1.1 \%$ & $0.5 \%$ \\
\hline Saddle Point 4 & $63.0 \%$ & $53.7 \%$ & $46.1 \%$ & $29.1 \%$ & $16.1 \%$ & $12.6 \%$ & $9.0 \%$ & $5.4 \%$ & $4.6 \%$ & $2.4 \%$ & $1.3 \%$ & $1.1 \%$ & $0.5 \%$ \\
\hline Large Dev & $71.0 \%$ & $57.0 \%$ & $47.6 \%$ & $24.2 \%$ & $13.6 \%$ & $10.7 \%$ & $7.7 \%$ & $4.7 \%$ & $4.0 \%$ & $2.1 \%$ & $1.2 \%$ & $0.9 \%$ & $0.4 \%$ \\
\hline Normal & $62.8 \%$ & $53.9 \%$ & $46.3 \%$ & $29.7 \%$ & $16.2 \%$ & $12.5 \%$ & $8.9 \%$ & $5.3 \%$ & $4.5 \%$ & $2.3 \%$ & $1.3 \%$ & $1.0 \%$ & $0.4 \%$ \\
\hline Jarrow-Rudd 3 & $62.7 \%$ & $53.6 \%$ & $46.0 \%$ & $29.4 \%$ & $16.2 \%$ & $12.7 \%$ & $9.1 \%$ & $5.5 \%$ & $4.6 \%$ & $2.4 \%$ & $1.3 \%$ & $1.1 \%$ & $0.5 \%$ \\
\hline Jarrow-Rudd 4 & $61.6 \%$ & $53.1 \%$ & $45.9 \%$ & $30.1 \%$ & $16.7 \%$ & $13.0 \%$ & $9.3 \%$ & $5.5 \%$ & $4.6 \%$ & $2.4 \%$ & $1.3 \%$ & $1.0 \%$ & $0.5 \%$ \\
\hline \multicolumn{14}{|l|}{ rho $=20 \%$} \\
\hline tranche & $0 \%-2 \%$ & $0 \%-3 \%$ & $0 \%-4 \%$ & $2 \%-4 \%$ & $3 \%-6 \%$ & $4 \%-6 \%$ & $4 \%-8 \%$ & $6 \%-8 \%$ & $6 \%-9 \%$ & $8 \%-10 \%$ & $9 \%-12 \%$ & $10 \%-12 \%$ & $12 \%-14 \%$ \\
\hline Recursion & $53.6 \%$ & $45.8 \%$ & $39.8 \%$ & $26.0 \%$ & $16.5 \%$ & $13.9 \%$ & $11.0 \%$ & $8.0 \%$ & $7.1 \%$ & $4.8 \%$ & $3.3 \%$ & $2.9 \%$ & $1.8 \%$ \\
\hline Saddle Point 2 & $53.0 \%$ & $45.5 \%$ & $39.6 \%$ & $26.2 \%$ & $16.7 \%$ & $14.1 \%$ & $11.1 \%$ & $8.1 \%$ & $7.2 \%$ & $4.8 \%$ & $3.4 \%$ & $3.0 \%$ & $1.9 \%$ \\
\hline Saddle Point 4 & $53.3 \%$ & $45.7 \%$ & $39.7 \%$ & $26.2 \%$ & $16.6 \%$ & $14.0 \%$ & $11.0 \%$ & $8.0 \%$ & $7.1 \%$ & $4.8 \%$ & $3.4 \%$ & $2.9 \%$ & $1.9 \%$ \\
\hline Large Dev & $60.0 \%$ & $48.7 \%$ & $41.3 \%$ & $22.7 \%$ & $14.7 \%$ & $12.4 \%$ & $9.9 \%$ & $7.3 \%$ & $6.5 \%$ & $4.4 \%$ & $3.1 \%$ & $2.7 \%$ & $1.7 \%$ \\
\hline Normal & $53.2 \%$ & $45.8 \%$ & $39.8 \%$ & $26.4 \%$ & $16.7 \%$ & $14.0 \%$ & $11.0 \%$ & $8.0 \%$ & $7.1 \%$ & $4.7 \%$ & $3.3 \%$ & $2.9 \%$ & $1.8 \%$ \\
\hline Jarrow-Rudd 3 & $53.1 \%$ & $45.6 \%$ & $39.7 \%$ & $26.3 \%$ & $16.7 \%$ & $14.1 \%$ & $11.1 \%$ & $8.0 \%$ & $7.1 \%$ & $4.8 \%$ & $3.4 \%$ & $2.9 \%$ & $1.9 \%$ \\
\hline Jarrow-Rudd 4 & $51.9 \%$ & $45.2 \%$ & $39.5 \%$ & $27.2 \%$ & $17.1 \%$ & $14.3 \%$ & $11.2 \%$ & $8.1 \%$ & $7.2 \%$ & $4.8 \%$ & $3.4 \%$ & $2.9 \%$ & $1.8 \%$ \\
\hline \multicolumn{14}{|l|}{ rho $=30 \%$} \\
\hline tranche & $0 \%-2 \%$ & $0 \%-3 \%$ & $0 \%-4 \%$ & $2 \%-4 \%$ & $3 \%-6 \%$ & $4 \%-6 \%$ & $4 \%-8 \%$ & $6 \%-8 \%$ & $6 \%-9 \%$ & $8 \%-10 \%$ & $9 \%-12 \%$ & $10 \%-12 \%$ & $12 \%-14 \%$ \\
\hline Recursion & $45.4 \%$ & $39.0 \%$ & $34.3 \%$ & $23.1 \%$ & $15.9 \%$ & $13.9 \%$ & $11.5 \%$ & $9.1 \%$ & $8.3 \%$ & $6.2 \%$ & $4.8 \%$ & $4.4 \%$ & $3.1 \%$ \\
\hline Saddle Point 2 & $44.9 \%$ & $38.8 \%$ & $34.1 \%$ & $23.3 \%$ & $16.0 \%$ & $14.0 \%$ & $\%$ & $9.1 \%$ & $8.4 \%$ & $6.2 \%$ & $4.8 \%$ & $4.4 \%$ & $3.1 \%$ \\
\hline Saddle Point 4 & $45.1 \%$ & $38.9 \%$ & $34.2 \%$ & $23.3 \%$ & $16.0 \%$ & $14.0 \%$ & $6 \%$ & $9.1 \%$ & $8.3 \%$ & $6.2 \%$ & $4.8 \%$ & $4.4 \%$ & $3.1 \%$ \\
\hline Large Dev & $50.7 \%$ & $41.6 \%$ & $35.7 \%$ & $20.7 \%$ & $14.5 \%$ & $12.8 \%$ & $10.6 \%$ & $8.5 \%$ & $7.8 \%$ & $5.8 \%$ & $4.5 \%$ & $4.1 \%$ & $3.0 \%$ \\
\hline Normal & $45.1 \%$ & $39.0 \%$ & $34.2 \%$ & $23.4 \%$ & $16.0 \%$ & $14.0 \%$ & $11.5 \%$ & $9.1 \%$ & $8.3 \%$ & $6.2 \%$ & $4.8 \%$ & $4.4 \%$ & $3.1 \%$ \\
\hline Jarrow-Rudd 3 & $45.0 \%$ & $38.9 \%$ & $34.2 \%$ & $23.4 \%$ & $16.0 \%$ & $14.0 \%$ & $11.6 \%$ & $9.1 \%$ & $8.3 \%$ & $6.2 \%$ & $4.8 \%$ & $4.4 \%$ & $3.1 \%$ \\
\hline Jarrow-Rudd 4 & $44.0 \%$ & $38.5 \%$ & $34.0 \%$ & $24.1 \%$ & $16.3 \%$ & $14.2 \%$ & $11.7 \%$ & $9.2 \%$ & $8.4 \%$ & $6.2 \%$ & $4.8 \%$ & $4.4 \%$ & $3.1 \%$ \\
\hline \multicolumn{14}{|l|}{ rho $=50 \%$} \\
\hline tranche & $0 \%-2 \%$ & $0 \%-3 \%$ & $0 \%-4 \%$ & $2 \%-4 \%$ & $3 \%-6 \%$ & $4 \%-6 \%$ & $4 \%-8 \%$ & $6 \%-8 \%$ & $6 \%-9 \%$ & $8 \%-10 \%$ & $9 \%-12 \%$ & $10 \%-12 \%$ & $12 \%-14 \%$ \\
\hline Recursion & $31.6 \%$ & $27.6 \%$ & $24.7 \%$ & $17.7 \%$ & $13.5 \%$ & $12.3 \%$ & $10.7 \%$ & $9.2 \%$ & $8.7 \%$ & $7.2 \%$ & $6.1 \%$ & $5.7 \%$ & $4.7 \%$ \\
\hline Saddle Point 2 & $31.4 \%$ & $27.5 \%$ & $24.6 \%$ & $17.9 \%$ & $13.6 \%$ & $12.3 \%$ & $10.8 \%$ & $9.2 \%$ & $8.7 \%$ & $7.2 \%$ & $6.1 \%$ & $5.8 \%$ & $4.7 \%$ \\
\hline Saddle Point 4 & $31.5 \%$ & $27.5 \%$ & $24.6 \%$ & $17.8 \%$ & $13.5 \%$ & $12.3 \%$ & $10.8 \%$ & $9.2 \%$ & $8.7 \%$ & $7.2 \%$ & $6.1 \%$ & $5.8 \%$ & $4.7 \%$ \\
\hline Large Dev & $35.3 \%$ & $29.5 \%$ & $25.8 \%$ & $16.4 \%$ & $12.6 \%$ & $11.5 \%$ & $10.2 \%$ & $8.8 \%$ & $8.3 \%$ & $6.8 \%$ & $5.9 \%$ & $5.6 \%$ & $4.5 \%$ \\
\hline Normal & $31.5 \%$ & $27.6 \%$ & $24.7 \%$ & $17.8 \%$ & $13.5 \%$ & $12.3 \%$ & $10.8 \%$ & $9.2 \%$ & $8.7 \%$ & $7.2 \%$ & $6.1 \%$ & $5.7 \%$ & $4.7 \%$ \\
\hline Jarrow-Rudd 3 & $31.4 \%$ & $27.5 \%$ & $24.6 \%$ & $17.9 \%$ & $13.5 \%$ & $12.3 \%$ & 10 & $9.2 \%$ & $8.7 \%$ & $7.2 \%$ & $6.1 \%$ & $5.8 \%$ & $4.7 \%$ \\
\hline Jarrow-Rudd 4 & $30.7 \%$ & $27.3 \%$ & $24.6 \%$ & $18.4 \%$ & $13.7 \%$ & $12.4 \%$ & $10.8 \%$ & $9.2 \%$ & $8.7 \%$ & $7.2 \%$ & $6.1 \%$ & $5.8 \%$ & $4.7 \%$ \\
\hline \multicolumn{14}{|l|}{ rho $=60 \%$} \\
\hline tranche & $0 \%-2 \%$ & $0 \%-3 \%$ & $0 \%-4 \%$ & $2 \%-4 \%$ & $3 \%-6 \%$ & $4 \%-6 \%$ & $4 \%-8 \%$ & $6 \%-8 \%$ & $6 \%-9 \%$ & $8 \%-10 \%$ & $9 \%-12 \%$ & $10 \%-12 \%$ & $12 \%-14 \%$ \\
\hline Recursion & $25.6 \%$ & $22.6 \%$ & $20.4 \%$ & $15.1 \%$ & $11.9 \%$ & $11.0 \%$ & $9.9 \%$ & $8.7 \%$ & $8.2 \%$ & $7.1 \%$ & $6.2 \%$ & $5.9 \%$ & $5.0 \%$ \\
\hline Saddle Point 2 & $25.4 \%$ & $22.5 \%$ & $20.3 \%$ & $15.3 \%$ & $12.0 \%$ & $11.1 \%$ & $9.9 \%$ & $8.7 \%$ & $8.2 \%$ & $7.1 \%$ & $6.2 \%$ & $5.9 \%$ & $5.0 \%$ \\
\hline Saddle Point 4 & $25.5 \%$ & $22.5 \%$ & $20.3 \%$ & $15.2 \%$ & $12.0 \%$ & $11.1 \%$ & $9.9 \%$ & $8.7 \%$ & $8.3 \%$ & $7.1 \%$ & $6.2 \%$ & $5.9 \%$ & $5.0 \%$ \\
\hline Large Dev & $28.7 \%$ & $24.2 \%$ & $21.4 \%$ & $14.1 \%$ & $11.3 \%$ & $10.4 \%$ & $9.4 \%$ & $8.4 \%$ & $7.9 \%$ & $6.7 \%$ & $6.0 \%$ & $5.8 \%$ & $4.9 \%$ \\
\hline Normal & $25.5 \%$ & $22.5 \%$ & $20.4 \%$ & $15.2 \%$ & $12.0 \%$ & $11.1 \%$ & $9.9 \%$ & $8.7 \%$ & $8.2 \%$ & $7.1 \%$ & $6.2 \%$ & $5.9 \%$ & $5.0 \%$ \\
\hline Jarrow-Rudd 3 & $25.4 \%$ & $22.5 \%$ & $20.3 \%$ & $15.2 \%$ & $12.0 \%$ & $11.1 \%$ & $9.9 \%$ & $8.7 \%$ & $8.3 \%$ & $7.1 \%$ & $6.2 \%$ & $5.9 \%$ & $5.0 \%$ \\
\hline Jarrow-Rudd 4 & $24.9 \%$ & $22.4 \%$ & $20.3 \%$ & $15.6 \%$ & $12.1 \%$ & $11.2 \%$ & $9.9 \%$ & $8.7 \%$ & $8.3 \%$ & $7.1 \%$ & $6.2 \%$ & $5.9 \%$ & $5.0 \%$ \\
\hline \multicolumn{14}{|l|}{ rho $=70 \%$} \\
\hline tranche & $0 \%-2 \%$ & $0 \%-3 \%$ & $0 \%-4 \%$ & $2 \%-4 \%$ & $3 \%-6 \%$ & $4 \%-6 \%$ & $4 \%-8 \%$ & $6 \%-8 \%$ & $6 \%-9 \%$ & $8 \%-10 \%$ & $9 \%-12 \%$ & $10 \%-12 \%$ & $12 \%-14 \%$ \\
\hline Recursion & $20.0 \%$ & $17.8 \%$ & $16.3 \%$ & $12.5 \%$ & $10.3 \%$ & $9.6 \%$ & $8.7 \%$ & $7.8 \%$ & $7.5 \%$ & $6.6 \%$ & $6.0 \%$ & $5.8 \%$ & $5.1 \%$ \\
\hline Saddle Point 2 & $19.9 \%$ & $17.7 \%$ & $16.2 \%$ & $12.6 \%$ & $10.3 \%$ & $9.7 \%$ & $8.7 \%$ & $7.8 \%$ & $7.5 \%$ & $6.7 \%$ & $6.0 \%$ & $5.8 \%$ & $5.1 \%$ \\
\hline Saddle Point 4 & $19.9 \%$ & $17.8 \%$ & $16.2 \%$ & $12.6 \%$ & $10.3 \%$ & $9.6 \%$ & $8.7 \%$ & $7.9 \%$ & $7.5 \%$ & $6.6 \%$ & $6.0 \%$ & $5.8 \%$ & $5.1 \%$ \\
\hline Large Dev & $39.2 \%$ & $30.3 \%$ & $25.3 \%$ & $11.4 \%$ & $9.4 \%$ & $9.0 \%$ & $8.1 \%$ & $7.2 \%$ & $6.9 \%$ & $6.1 \%$ & $5.5 \%$ & $5.3 \%$ & $4.6 \%$ \\
\hline Normal & $19.9 \%$ & $17.8 \%$ & $16.2 \%$ & $12.6 \%$ & $10.3 \%$ & $9.6 \%$ & $8.7 \%$ & $7.9 \%$ & $7.5 \%$ & $6.6 \%$ & $5.9 \%$ & $5.7 \%$ & $5.1 \%$ \\
\hline Jarrow-Rudd 3 & $19.8 \%$ & $17.8 \%$ & $16.2 \%$ & $12.6 \%$ & $10.3 \%$ & $9.6 \%$ & $8.8 \%$ & $7.9 \%$ & $7.5 \%$ & $6.6 \%$ & $6.0 \%$ & $5.8 \%$ & $5.1 \%$ \\
\hline Jarrow-Rudd 4 & $19.5 \%$ & $17.7 \%$ & $16.2 \%$ & $12.9 \%$ & $10.4 \%$ & $9.7 \%$ & $8.8 \%$ & $7.9 \%$ & $7.6 \%$ & $6.6 \%$ & $6.0 \%$ & $5.8 \%$ & $5.1 \%$ \\
\hline
\end{tabular}


The expected loss relative difference with the recursion (in percentage) for each tranche is given by:

\begin{tabular}{|c|c|c|c|c|c|c|c|c|c|c|c|c|c|}
\hline tranche & $0 \%-2 \%$ & $0 \%-3 \%$ & $0 \%-4 \%$ & $2 \%-4 \%$ & $3 \%-6 \%$ & $4 \%-6 \%$ & $4 \%-8 \%$ & $6 \%-8 \%$ & $6 \%-9 \%$ & $8 \%-10 \%$ & $9 \%-12 \%$ & $10 \%-12 \%$ & $12 \%-14 \%$ \\
\hline Saddle Point 2 & $0.9 \%$ & $1.2 \%$ & $0.9 \%$ & $0.8 \%$ & $4.5 \%$ & $8.0 \%$ & $8.8 \%$ & $12.9 \%$ & $13.1 \%$ & $14.6 \%$ & $14.9 \%$ & $15.2 \%$ & $17.1 \%$ \\
\hline Saddle Point 4 & $0.8 \%$ & $0.5 \%$ & $0.3 \%$ & $0.7 \%$ & $1.9 \%$ & $2.8 \%$ & $3.2 \%$ & $5.4 \%$ & $5.5 \%$ & $7.4 \%$ & $8.2 \%$ & $9.0 \%$ & $12.2 \%$ \\
\hline Large Dev & $12.4 \%$ & $5.1 \%$ & $1.8 \%$ & $23.5 \%$ & $23.4 \%$ & $20.4 \%$ & $18.9 \%$ & $11.4 \%$ & $10.9 \%$ & $6.5 \%$ & $4.9 \%$ & $4.1 \%$ & $2.4 \%$ \\
\hline Normal & $1.8 \%$ & $0.0 \%$ & $0.7 \%$ & $6.6 \%$ & $2.3 \%$ & $2.5 \%$ & $5.6 \%$ & $21.6 \%$ & $23.3 \%$ & $41.0 \%$ & $50.5 \%$ & $56.9 \%$ & $68.3 \%$ \\
\hline Jarrow-Rudd 3 & $1.4 \%$ & $0.8 \%$ & $0.5 \%$ & $1.7 \%$ & $3.6 \%$ & $5.1 \%$ & $5.2 \%$ & $5.6 \%$ & $5.1 \%$ & $0.9 \%$ & $7.4 \%$ & $12.2 \%$ & $24.4 \%$ \\
\hline Jarrow-Rudd 4 & $1.9 \%$ & $1.1 \%$ & $0.7 \%$ & $2.1 \%$ & $4.3 \%$ & $7.0 \%$ & $7.8 \%$ & $12.0 \%$ & $11.5 \%$ & $5.8 \%$ & $2.0 \%$ & $8.5 \%$ & $24.4 \%$ \\
\hline \multicolumn{14}{|l|}{ rho $=10 \%$} \\
\hline tranche & $0 \%-2 \%$ & $0 \%-3 \%$ & $0 \%-4 \%$ & $2 \%-4 \%$ & $3 \%-6 \%$ & $4 \%-6 \%$ & $4 \%-8 \%$ & $6 \%-8 \%$ & $6 \%-9 \%$ & $8 \%-10 \%$ & 9\%-12\% & $10 \%-12 \%$ & $12 \%-14 \%$ \\
\hline Saddle Point 2 & $1.1 \%$ & $0.8 \%$ & $0.5 \%$ & $0.9 \%$ & $1.6 \%$ & $1.9 \%$ & $2.0 \%$ & $2.3 \%$ & $2.4 \%$ & $2.6 \%$ & $2.6 \%$ & $2.7 \%$ & $2.7 \%$ \\
\hline Saddle Point 4 & $0.7 \%$ & $0.4 \%$ & $0.3 \%$ & $0.8 \%$ & $1.0 \%$ & $1.0 \%$ & $1.1 \%$ & $1.1 \%$ & $1.1 \%$ & $1.2 \%$ & $1.2 \%$ & $1.2 \%$ & $1.2 \%$ \\
\hline Large Dev & $11.8 \%$ & $5.8 \%$ & $3.1 \%$ & $16.1 \%$ & $15.0 \%$ & $14.1 \%$ & $13.5 \%$ & $12.1 \%$ & $11.9 \%$ & $11.2 \%$ & $10.3 \%$ & $9.4 \%$ & $8.4 \%$ \\
\hline Normal & $1.1 \%$ & $0.1 \%$ & $0.2 \%$ & $2.9 \%$ & $1.3 \%$ & $0.6 \%$ & $0.0 \%$ & $1.3 \%$ & $1.6 \%$ & $2.8 \%$ & $3.5 \%$ & $3.9 \%$ & $4.7 \%$ \\
\hline Jarrow-Rudd 3 & $1.2 \%$ & $0.6 \%$ & $0.4 \%$ & $1.6 \%$ & $1.6 \%$ & $1.6 \%$ & $1.6 \%$ & $1.4 \%$ & $1.4 \%$ & $1.2 \%$ & $1.1 \%$ & $1.1 \%$ & $0.9 \%$ \\
\hline Jarrow-Rudd 4 & $2.9 \%$ & $1.5 \%$ & $0.7 \%$ & $4.1 \%$ & $4.2 \%$ & $4.0 \%$ & $3.7 \%$ & $2.8 \%$ & $2.5 \%$ & $1.4 \%$ & $0.5 \%$ & $0.2 \%$ & $0.9 \%$ \\
\hline \multicolumn{14}{|l|}{$\mathrm{rho}=20 \%$} \\
\hline tranche & $0 \%-2 \%$ & $0 \%-3 \%$ & $0 \%-4 \%$ & $2 \%-4 \%$ & $3 \%-6 \%$ & $4 \%-6 \%$ & $4 \%-8 \%$ & $6 \%-8 \%$ & $6 \%-9 \%$ & $8 \%-10 \%$ & $9 \%-12 \%$ & $10 \%-12 \%$ & $12 \%-14 \%$ \\
\hline Saddle Point 2 & $1.1 \%$ & $0.6 \%$ & $0.4 \%$ & $1.0 \%$ & $1.1 \%$ & $1.1 \%$ & $1.0 \%$ & $0.9 \%$ & $0.8 \%$ & $0.8 \%$ & $0.9 \%$ & $0.7 \%$ & $0.8 \%$ \\
\hline Saddle Point 4 & $0.7 \%$ & $0.4 \%$ & $0.2 \%$ & $0.7 \%$ & $0.6 \%$ & $0.6 \%$ & $0.6 \%$ & $0.5 \%$ & $0.5 \%$ & $0.4 \%$ & $\%$ & $0.4 \%$ & $0.4 \%$ \\
\hline Large Dev & $11.9 \%$ & $6.3 \%$ & $3.9 \%$ & $12.7 \%$ & $11.1 \%$ & $10.8 \%$ & $10.0 \%$ & $8.7 \%$ & $8.5 \%$ & $7.8 \%$ & $7.5 \%$ & $7.2 \%$ & $5.9 \%$ \\
\hline Normal & $0.8 \%$ & $0.2 \%$ & $0.0 \%$ & $1.6 \%$ & $0.8 \%$ & $0.5 \%$ & $0.3 \%$ & $0.1 \%$ & $0.1 \%$ & $0.4 \%$ & $0.5 \%$ & $0.6 \%$ & $0.7 \%$ \\
\hline Jarrow-Rudd 3 & $1.0 \%$ & $0.5 \%$ & $0.3 \%$ & $1.3 \%$ & $1.0 \%$ & $0.9 \%$ & $0.8 \%$ & $0.7 \%$ & $0.6 \%$ & $0.5 \%$ & $0.5 \%$ & $0.5 \%$ & $0.4 \%$ \\
\hline Jarrow-Rudd 4 & $3.2 \%$ & $1.4 \%$ & $0.7 \%$ & $4.6 \%$ & $3.1 \%$ & $2.6 \%$ & $2.2 \%$ & $1.5 \%$ & $1.4 \%$ & $0.9 \%$ & $0.6 \%$ & $0.4 \%$ & $0.2 \%$ \\
\hline \multicolumn{14}{|l|}{ rho $=30 \%$} \\
\hline tranche & $0 \%-2 \%$ & $0 \%-3 \%$ & $0 \%-4 \%$ & $2 \%-4 \%$ & $3 \%-6 \%$ & $4 \%-6 \%$ & $4 \%-8 \%$ & $6 \%-8 \%$ & $6 \%-9 \%$ & $8 \%-10 \%$ & 9\%-12\% & $10 \%-12 \%$ & $12 \%-14 \%$ \\
\hline Saddle Point 2 & $1.0 \%$ & $0.5 \%$ & $0.3 \%$ & $0.9 \%$ & $0.7 \%$ & $0.7 \%$ & $0.6 \%$ & $0.5 \%$ & $0.6 \%$ & $0.7 \%$ & $0.4 \%$ & $0.2 \%$ & $0.3 \%$ \\
\hline Saddle Point 4 & $0.6 \%$ & $0.3 \%$ & $0.2 \%$ & $0.7 \%$ & $0.5 \%$ & $0.4 \%$ & $0.4 \%$ & $0.3 \%$ & $0.3 \%$ & $0.3 \%$ & $0.2 \%$ & $0.2 \%$ & $0.2 \%$ \\
\hline Large Dev & $11.6 \%$ & $6.5 \%$ & $4.2 \%$ & $10.3 \%$ & $8.8 \%$ & $8.5 \%$ & $7.8 \%$ & $6.8 \%$ & $6.7 \%$ & $5.8 \%$ & $5.6 \%$ & $6.1 \%$ & $4.1 \%$ \\
\hline Normal & $0.6 \%$ & $0.2 \%$ & $0.0 \%$ & $1.1 \%$ & $0.5 \%$ & $0.4 \%$ & $0.3 \%$ & $0.1 \%$ & $0.1 \%$ & $0.1 \%$ & $0.1 \%$ & $0.1 \%$ & $0.2 \%$ \\
\hline Jarrow-Rudd 3 & $0.9 \%$ & $0.4 \%$ & $0.3 \%$ & $1.1 \%$ & $0.7 \%$ & $0.6 \%$ & $0.5 \%$ & $0.4 \%$ & $0.4 \%$ & $0.3 \%$ & $0.3 \%$ & $0.3 \%$ & $0.2 \%$ \\
\hline Jarrow-Rudd 4 & $3.2 \%$ & $1.2 \%$ & $0.6 \%$ & $4.5 \%$ & $2.4 \%$ & $1.9 \%$ & $1.5 \%$ & $1.0 \%$ & $0.9 \%$ & $0.6 \%$ & $0.4 \%$ & $0.4 \%$ & $0.2 \%$ \\
\hline \multicolumn{14}{|l|}{ rho $=50 \%$} \\
\hline tranche & $0 \%-2 \%$ & $0 \%-3 \%$ & $0 \%-4 \%$ & $2 \%-4 \%$ & $3 \%-6 \%$ & $4 \%-6 \%$ & $4 \%-8 \%$ & $6 \%-8 \%$ & $6 \%-9 \%$ & $8 \%-10 \%$ & $9 \%-12 \%$ & $10 \%-12 \%$ & $12 \%-14 \%$ \\
\hline Saddle Point 2 & $0.8 \%$ & $0.5 \%$ & $0.2 \%$ & $0.9 \%$ & $0.6 \%$ & $0.2 \%$ & $0.3 \%$ & $0.4 \%$ & $0.2 \%$ & $0.1 \%$ & $0.6 \%$ & $0.6 \%$ & $0.5 \%$ \\
\hline Saddle Point 4 & $0.5 \%$ & $3 \%$ & $\%$ & $.5 \%$ & $0.3 \%$ & $0.3 \%$ & $0.2 \%$ & $0.2 \%$ & $0.2 \%$ & $0.1 \%$ & $0.1 \%$ & $0.1 \%$ & $0.1 \%$ \\
\hline Large Dev & $11.7 \%$ & $7.0 \%$ & $4.7 \%$ & $7.7 \%$ & $6.4 \%$ & $6.1 \%$ & $5.1 \%$ & $3.8 \%$ & $4.7 \%$ & $5.6 \%$ & $3.4 \%$ & $2.8 \%$ & $4.0 \%$ \\
\hline Normal & $0.5 \%$ & $0.2 \%$ & $0.1 \%$ & $0.7 \%$ & $0.3 \%$ & $0.2 \%$ & $0.2 \%$ & $0.1 \%$ & $0.1 \%$ & $0.1 \%$ & $0.0 \%$ & $0.0 \%$ & $0.0 \%$ \\
\hline Jarrow-Rudd 3 & $0.8 \%$ & $0.4 \%$ & $0.2 \%$ & $0.8 \%$ & $0.4 \%$ & $0.4 \%$ & $0.3 \%$ & $0.2 \%$ & $0.2 \%$ & $0.2 \%$ & $0.1 \%$ & $0.1 \%$ & $0.1 \%$ \\
\hline Jarrow-Rudd 4 & $2.8 \%$ & $1.1 \%$ & $0.5 \%$ & $3.7 \%$ & $1.6 \%$ & $1.2 \%$ & $0.9 \%$ & $0.6 \%$ & $0.5 \%$ & $0.4 \%$ & $0.2 \%$ & $0.2 \%$ & $0.2 \%$ \\
\hline \multicolumn{14}{|l|}{ rho $=60 \%$} \\
\hline tranche & $0 \%-2 \%$ & $0 \%-3 \%$ & $0 \%-4 \%$ & $2 \%-4 \%$ & $3 \%-6 \%$ & $4 \%-6 \%$ & $4 \%-8 \%$ & $6 \%-8 \%$ & $6 \%-9 \%$ & $8 \%-10 \%$ & $9 \%-12 \%$ & $10 \%-12 \%$ & $12 \%-14 \%$ \\
\hline Saddle Point 2 & $0.9 \%$ & $0.3 \%$ & $0.2 \%$ & $0.8 \%$ & $0.4 \%$ & $0.5 \%$ & $0.3 \%$ & $0.1 \%$ & $0.1 \%$ & $0.2 \%$ & $0.6 \%$ & $0.6 \%$ & $0.3 \%$ \\
\hline Saddle Point 4 & $0.5 \%$ & $0.2 \%$ & & $0.5 \%$ & $0.3 \%$ & $0.2 \%$ & $0.2 \%$ & $0.2 \%$ & $0.1 \%$ & $0.1 \%$ & $0.1 \%$ & $0.1 \%$ & $0.1 \%$ \\
\hline Large Dev & $12.1 \%$ & $7.1 \%$ & & $6.9 \%$ & $5.7 \%$ & $6.1 \%$ & $4.9 \%$ & $3.3 \%$ & $4.0 \%$ & $4.6 \%$ & $2.7 \%$ & $2.3 \%$ & $2.1 \%$ \\
\hline Normal & $0.5 \%$ & $0.2 \%$ & $0.1 \%$ & $0.6 \%$ & $0.3 \%$ & $0.2 \%$ & $0.2 \%$ & $0.1 \%$ & $0.1 \%$ & $0.1 \%$ & $0.0 \%$ & $0.1 \%$ & $0.0 \%$ \\
\hline Jarrow-Rudd 3 & $0.7 \%$ & $0.3 \%$ & $0.2 \%$ & $0.7 \%$ & $0.3 \%$ & $0.3 \%$ & $0.3 \%$ & $0.2 \%$ & $0.2 \%$ & $0.1 \%$ & $0.1 \%$ & $0.1 \%$ & $0.1 \%$ \\
\hline Jarrow-Rudd 4 & $2.6 \%$ & $0.9 \%$ & $0.5 \%$ & $3.1 \%$ & $1.3 \%$ & $1.0 \%$ & $0.8 \%$ & $0.5 \%$ & $0.5 \%$ & $0.3 \%$ & $0.1 \%$ & $0.0 \%$ & $0.2 \%$ \\
\hline \multicolumn{14}{|l|}{ rho $=70 \%$} \\
\hline tranche & $0 \%-2 \%$ & $0 \%-3 \%$ & $0 \%-4 \%$ & $2 \%-4 \%$ & $3 \%-6 \%$ & $4 \%-6 \%$ & $4 \%-8 \%$ & $6 \%-8 \%$ & $6 \%-9 \%$ & $8 \%-10 \%$ & $9 \%-12 \%$ & $10 \%-12 \%$ & $12 \%-14 \%$ \\
\hline Saddle Point 2 & $0.6 \%$ & $0.4 \%$ & $0.3 \%$ & $0.2 \%$ & $0.7 \%$ & $0.9 \%$ & $0.2 \%$ & $0.7 \%$ & $0.3 \%$ & $0.6 \%$ & $0.5 \%$ & $0.5 \%$ & $0.2 \%$ \\
\hline Saddle Point 4 & $0.5 \%$ & $0.2 \%$ & $0.1 \%$ & $0.4 \%$ & $0.2 \%$ & $0.2 \%$ & $0.1 \%$ & $0.1 \%$ & $0.1 \%$ & $0.1 \%$ & $0.1 \%$ & $0.1 \%$ & $0.0 \%$ \\
\hline Large Dev & $96.4 \%$ & $69.9 \%$ & $55.7 \%$ & $9.2 \%$ & $8.2 \%$ & $6.9 \%$ & $7.8 \%$ & $8.8 \%$ & $8.7 \%$ & $8.0 \%$ & $7.7 \%$ & $7.8 \%$ & $8.5 \%$ \\
\hline Normal & $0.4 \%$ & $0.2 \%$ & $0.1 \%$ & $0.5 \%$ & $0.2 \%$ & $0.0 \%$ & $0.2 \%$ & $0.4 \%$ & $0.3 \%$ & $0.0 \%$ & $0.3 \%$ & $0.4 \%$ & $0.1 \%$ \\
\hline Jarrow-Rudd 3 & $0.6 \%$ & $0.3 \%$ & $0.2 \%$ & $0.6 \%$ & $0.3 \%$ & $0.3 \%$ & $0.2 \%$ & $0.1 \%$ & $0.1 \%$ & $0.0 \%$ & $0.1 \%$ & $0.1 \%$ & $0.1 \%$ \\
\hline Jarrow-Rudd 4 & $2.2 \%$ & $0.9 \%$ & $0.4 \%$ & $2.6 \%$ & $1.1 \%$ & $0.4 \%$ & $0.6 \%$ & $0.8 \%$ & $0.6 \%$ & $0.0 \%$ & $0.1 \%$ & $0.1 \%$ & $0.3 \%$ \\
\hline
\end{tabular}

The PV01 for each tranche is given by: 


\begin{tabular}{|c|c|c|c|c|c|c|c|c|c|c|c|c|c|}
\hline tranche & $0 \%-2 \%$ & $0 \%-3 \%$ & $0 \%-4 \%$ & $2 \%-4 \%$ & $3 \%-6 \%$ & $4 \%-6 \%$ & $4 \%-8 \%$ & $6 \%-8 \%$ & $6 \%-9 \%$ & $8 \%-10 \%$ & $9 \%-12 \%$ & $10 \%-12 \%$ & $12 \%-14 \%$ \\
\hline Recursion & 2.46 & 2.93 & 3.27 & 4.08 & 4.45 & 4.53 & 4.58 & 4.62 & 4.62 & 4.63 & 4.64 & 4.64 & 4.64 \\
\hline Saddle Point 2 & 2.50 & 2.95 & 3.28 & 4.05 & 4.43 & 4.52 & 4.57 & 4.62 & 4.62 & 4.63 & 4.64 & 4.64 & 4.64 \\
\hline Saddle Point 4 & 2.48 & 2.94 & 3.27 & 4.07 & 4.44 & 4.53 & 4.57 & 4.62 & 4.62 & 4.63 & 4.64 & 4.64 & 4.64 \\
\hline Large Dev & 2.28 & 2.89 & 3.26 & 4.23 & 4.49 & 4.55 & 4.59 & 4.62 & 4.63 & 4.63 & 4.64 & 4.64 & 4.64 \\
\hline Normal & 2.46 & 2.92 & 3.26 & 4.06 & 4.46 & 4.54 & 4.58 & 4.63 & 4.63 & 4.64 & 4.64 & 4.64 & 4.64 \\
\hline Jarrow-Rudd 3 & 2.49 & 2.94 & 3.27 & 4.05 & 4.44 & 4.52 & 4.57 & 4.62 & 4.62 & 4.63 & 4.64 & 4.64 & 4.64 \\
\hline Jarrow-Rudd 4 & 2.54 & 2.96 & 3.28 & 4.01 & 4.42 & 4.51 & 4.56 & 4.62 & 4.62 & 4.63 & 4.64 & 4.64 & 4.64 \\
\hline
\end{tabular}

\begin{tabular}{|c|c|c|c|c|c|c|c|c|c|c|c|c|c|}
\hline rho $=10 \%$ & & & & & & & & & & & & & \\
\hline tranche & $0 \%-2 \%$ & $0 \%-3 \%$ & $0 \%-4 \%$ & $2 \%-4 \%$ & $3 \%-6 \%$ & $4 \%-6 \%$ & $4 \%-8 \%$ & $6 \%-8 \%$ & $6 \%-9 \%$ & $8 \%-10 \%$ & $9 \%-12 \%$ & $10 \%-12 \%$ & $12 \%-14 \%$ \\
\hline Recursion & 2.73 & 3.11 & 3.38 & 4.03 & 4.34 & 4.42 & 4.49 & 4.56 & 4.57 & 4.60 & 4.62 & 4.62 & 4.63 \\
\hline Saddle Point 2 & 2.76 & 3.12 & 3.39 & 4.01 & 4.33 & 4.42 & 4.48 & 4.55 & 4.57 & 4.60 & 4.62 & 4.62 & 4.63 \\
\hline Saddle Point 4 & 2.75 & 3.12 & 3.38 & 4.02 & 4.34 & 4.42 & 4.49 & 4.56 & 4.57 & 4.60 & 4.62 & 4.62 & 4.63 \\
\hline Large Dev & 2.57 & 3.05 & 3.36 & 4.14 & 4.39 & 4.46 & 4.51 & 4.57 & 4.58 & 4.61 & 4.62 & 4.62 & 4.63 \\
\hline Normal & 2.74 & 3.11 & 3.38 & 4.01 & 4.34 & 4.43 & 4.49 & 4.56 & 4.57 & 4.61 & 4.62 & 4.62 & 4.63 \\
\hline Jarrow-Rudd 3 & 2.76 & 3.12 & 3.38 & 4.01 & 4.34 & 4.42 & 4.49 & 4.55 & 4.57 & 4.60 & 4.62 & 4.62 & 4.63 \\
\hline Jarrow-Rudd 4 & 2.82 & 3.14 & 3.39 & 3.96 & 4.32 & 4.41 & 4.48 & 4.55 & 4.57 & 4.60 & 4.62 & 4.62 & 4.63 \\
\hline
\end{tabular}

\begin{tabular}{|c|c|c|c|c|c|c|c|c|c|c|c|c|c|}
\hline & & & & & & & & & & & & & \\
\hline tranche & $0 \%-2 \%$ & $0 \%-3 \%$ & $0 \%-4 \%$ & $2 \%-4 \%$ & $3 \%-6 \%$ & $4 \%-6 \%$ & $4 \%-8 \%$ & $6 \%-8 \%$ & $6 \%-9 \%$ & $8 \%-10 \%$ & $9 \%-12 \%$ & $10 \%-12 \%$ & $12 \%-14 \%$ \\
\hline Recursion & 3.02 & 3.32 & 3.53 & 4.03 & 4.29 & 4.35 & 4.42 & 4.49 & 4.51 & 4.55 & 4.58 & 4.59 & 4.61 \\
\hline Saddle Point 2 & 3.05 & 3.33 & 3.53 & 4.02 & 4.28 & 4.35 & 4.42 & 4.49 & 4.50 & 4.55 & 4.58 & 4.59 & 4.61 \\
\hline Saddle Point 4 & 3.04 & 3.32 & 3.53 & 4.02 & 4.28 & 4.35 & 4.42 & 4.49 & 4.50 & 4.55 & 4.58 & 4.59 & 4.61 \\
\hline Large Dev & 2.87 & 3.25 & 3.50 & 4.12 & 4.33 & 4.38 & 4.44 & 4.50 & 4.52 & 4.56 & 4.58 & 4.59 & 4.61 \\
\hline Normal & 3.03 & 3.32 & 3.53 & 4.02 & 4.28 & 4.35 & 4.42 & 4.49 & 4.51 & 4.55 & 4.58 & 4.59 & 4.61 \\
\hline Jarrow-Rudd 3 & 3.04 & 3.32 & 3.53 & 4.02 & 4.28 & 4.35 & 4.42 & 4.49 & 4.50 & 4.55 & 4.58 & 4.59 & 4.61 \\
\hline Jarrow-Rudd 4 & 3.09 & 3.34 & 3.53 & 3.98 & 4.27 & 4.34 & 4.41 & 4.48 & 4.50 & 4.55 & 4.58 & 4.59 & 4.61 \\
\hline
\end{tabular}

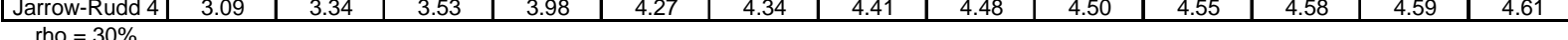

\begin{tabular}{|c|c|c|c|c|c|c|c|c|c|c|c|c|c|}
\hline tranche & $0 \%-2 \%$ & $0 \%-3 \%$ & $0 \%-4 \%$ & $2 \%-4 \%$ & $3 \%-6 \%$ & $4 \%-6 \%$ & $4 \%-8 \%$ & $6 \%-8 \%$ & $6 \%-9 \%$ & $8 \%-10 \%$ & $9 \%-12 \%$ & $10 \%-12 \%$ & $12 \%-14 \%$ \\
\hline Recursion & 3.27 & 3.51 & 3.67 & 4.07 & 4.27 & 4.32 & 4.38 & 4.45 & 4.46 & 4.51 & 4.54 & 4.55 & 4.58 \\
\hline Saddle Point 2 & 3.29 & 3.51 & 3.67 & 4.06 & 4.26 & 4.32 & 4.38 & 4.44 & 4.46 & 4.51 & 4.54 & 4.55 & 4.58 \\
\hline Saddle Point 4 & 3.28 & 3.51 & 3.67 & 4.06 & 4.27 & 4.32 & 4.38 & 4.44 & 4.46 & 4.51 & 4.54 & 4.55 & 4.58 \\
\hline Large Dev & 3.14 & 3.44 & 3.64 & 4.14 & 4.31 & 4.35 & 4.41 & 4.46 & 4.48 & 4.52 & 4.55 & 4.56 & 4.58 \\
\hline Normal & 3.28 & 3.51 & 3.67 & 4.06 & 4.27 & 4.32 & 4.38 & 4.45 & 4.46 & 4.51 & 4.54 & 4.55 & 4.58 \\
\hline Jarrow-Rudd 3 & 3.29 & 3.51 & 3.67 & 4.06 & 4.27 & 4.32 & 4.38 & 4.44 & 4.46 & 4.51 & 4.54 & 4.55 & 4.58 \\
\hline Jarrow-Rudd 4 & 3.33 & 3.52 & 3.67 & 4.02 & 4.26 & 4.32 & 4.38 & 4.44 & 4.46 & 4.51 & 4.54 & 4.55 & 4.58 \\
\hline
\end{tabular}

\begin{tabular}{|c|c|c|c|c|c|c|c|c|c|c|c|c|c|}
\hline tranche & $0 \%-2 \%$ & $0 \%-3 \%$ & $0 \%-4 \%$ & $2 \%-4 \%$ & $3 \%-6 \%$ & $4 \%-6 \%$ & $4 \%-8 \%$ & $6 \% 8 \%$ & $6 \%-9 \%$ & $8 \% 10 \%$ & $0 \% 12 \%$ & ו & $12 \% 14 \%$ \\
\hline Recursion & 3.70 & 3.84 & 3.93 & 4.17 & 4.30 & 4.33 & 4.37 & 442 & 443 & 47 & 150 & 151 & $12 \%-14 \%$ \\
\hline Saddle Point 2 & 3.71 & 3.84 & 3.94 & 4.16 & 4.29 & 433 & 4.37 & 442 & 10 & 17 & 450 & 151 & 4.53 \\
\hline Saddle Point 4 & 3.70 & 3.84 & 3.94 & 4.17 & 4.29 & 4.33 & 4.37 & 4.42 & 443 & 447 & 450 & 451 & 4.53 \\
\hline Large Dev & 3.60 & 3.79 & 3.90 & 4.21 & 4.32 & 4.35 & 4.39 & 4.43 & 4.44 & 4.48 & 4.50 & 4.51 & 4.54 \\
\hline Normal & 3.70 & 3.84 & 3.93 & 4.17 & 4.30 & 4.33 & 4.37 & 4.42 & 4.43 & 4.47 & 4.50 & 4.51 & 4.53 \\
\hline Jarrow-Rudd 3 & 3.71 & 3.84 & 3.94 & 4.17 & 4.29 & 4.33 & 4.37 & 4.42 & 4.43 & 4.47 & 4.50 & 4.51 & 4.53 \\
\hline Jarrow-Rudd 4 & 3.73 & 3.85 & 3.94 & 4.15 & 4.29 & 4.33 & 4.37 & 4.41 & 4.43 & 4.47 & 4.50 & 4.51 & 4.53 \\
\hline
\end{tabular}

\begin{tabular}{|c|c|c|c|c|c|c|c|c|c|c|c|c|c|}
\hline tranche & $0 \%-2 \%$ & $0 \%-3 \%$ & $0 \%-4 \%$ & $2 \%-4 \%$ & $3 \%-6 \%$ & $4 \%-6 \%$ & $4 \%-8 \%$ & $6 \%-8 \%$ & $6 \%-9 \%$ & $8 \%-10 \%$ & $9 \%-12 \%$ & $10 \%-12 \%$ & $12 \%-14 \%$ \\
\hline Recursion & 3.89 & 3.99 & 4.06 & 4.23 & 4.33 & 4.35 & 4.39 & 4.42 & 4.43 & 4.46 & 4.49 & 4.50 & 4.52 \\
\hline Saddle Point 2 & 3.89 & 3.99 & 4.06 & 4.23 & 4.33 & 4.35 & 4.39 & 4.42 & 4.43 & 4.46 & 4.49 & 4.50 & 4.52 \\
\hline Saddle Point 4 & 3.89 & 3.99 & 4.06 & 4.23 & 4.33 & 4.35 & 4.39 & 4.42 & 4.43 & 4.46 & 4.49 & 4.50 & 4.52 \\
\hline Large Dev & 3.80 & 3.94 & 4.03 & 4.26 & 4.35 & 4.37 & 4.40 & 4.43 & 4.44 & 4.47 & 4.49 & 4.50 & 4.52 \\
\hline Normal & 3.89 & 3.99 & 4.06 & 4.23 & 4.33 & 4.35 & 4.39 & 4.42 & 4.43 & 4.46 & 4.49 & 4.50 & 4.52 \\
\hline Jarrow-Rudd 3 & 3.89 & 3.99 & 4.06 & 4.23 & 4.33 & 4.35 & 4.39 & 4.42 & 4.43 & 4.46 & 4.49 & 4.50 & 4.52 \\
\hline Jarrow-Rudd 4 & 3.91 & 3.99 & 4.06 & 4.22 & 4.32 & 4.35 & 4.38 & 4.42 & 4.43 & 4.46 & 4.49 & 4.50 & 4.52 \\
\hline
\end{tabular}

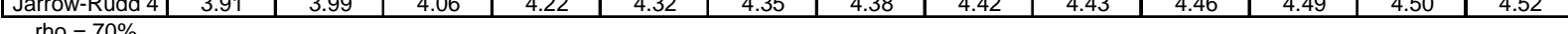

\begin{tabular}{|c|c|c|c|c|c|c|c|c|c|c|c|c|c|}
\hline tranche & $0 \%-2 \%$ & $0 \%-3 \%$ & $0 \%-4 \%$ & $2 \%-4 \%$ & $3 \%-6 \%$ & $4 \%-6 \%$ & $4 \%-8 \%$ & $6 \%-8 \%$ & $6 \%-9 \%$ & $8 \%-10 \%$ & $9 \%-12 \%$ & $10 \%-12 \%$ & $12 \%-14 \%$ \\
\hline Recursion & 4.06 & 4.13 & 4.18 & 4.30 & 4.37 & 4.39 & 4.41 & 4.44 & 4.44 & 4.47 & 4.49 & 4.49 & 4.51 \\
\hline Saddle Point 2 & 4.06 & 4.13 & 4.18 & 4.30 & 4.37 & 4.38 & 4.41 & 4.44 & 4.44 & 4.47 & 4.49 & 4.49 & 4.51 \\
\hline Saddle Point 4 & 4.06 & 4.13 & 4.18 & 4.30 & 4.37 & 4.39 & 4.41 & 4.44 & 4.44 & 4.47 & 4.49 & 4.49 & 4.51 \\
\hline Large Dev & 3.85 & 4.00 & 4.09 & 4.32 & 4.38 & 4.40 & 4.42 & 4.45 & 4.45 & 4.48 & 4.50 & 4.50 & 4.52 \\
\hline Normal & 4.06 & 4.13 & 4.18 & 4.30 & 4.37 & 4.39 & 4.41 & 4.44 & 4.44 & 4.47 & 4.49 & 4.49 & 4.51 \\
\hline Jarrow-Rudd 3 & 4.06 & 4.13 & 4.18 & 4.30 & 4.37 & 4.38 & 4.41 & 4.44 & 4.44 & 4.47 & 4.49 & 4.49 & 4.51 \\
\hline Jarrow-Rudd 4 & 4.07 & 4.13 & 4.18 & 4.29 & 4.36 & 4.38 & 4.41 & 4.44 & 4.44 & 4.47 & 4.49 & 4.49 & 4.51 \\
\hline
\end{tabular}

This quantity varies less that the spread as a function of the numerical method, as we can expect from a PV01. 


\section{The Esscher Transform}

The Esscher Transform is more often used in insurance than n Finance. It refers to a paper from F. Esscher, in 1932 (cf. [12]). As quoted in [15], "The Esscher transform was developed to approximate the aggregate claim amount distribution around a point of interest, $x_{0}$, by applying an analytic approximation (the Edgeworth series) to the transformed distribution with a parameter $\theta$ chosen such that the new mean is equal to $\hat{x}_{0}$. When the Esscher transform is used to calculate a stop-loss premium, the parameter $\theta$ is usually determined by specifying the mean of the transformed distribution as the retention limit." The Esscher Transform has an analogy in Finance with the Change of Measure, and the commonly used Change of Numeraire discovered by H. Geman, N. El Karoui, J.C. Rochet [14].

\section{I.1 General definition and analogy with a change of measure}

Let suppose that a random variable $X$ has a density function $f(x)$ in a probability space $\left(\Omega, F, F_{t}, Q\right)$. We define for $\theta \in \mathbb{R}$ :

$$
f_{\theta}(x)=\frac{e^{\theta x} f(x)}{M(\theta)} \text { and } M(\theta)=E\left(e^{\theta X}\right) .
$$

We check easily that $\int f_{\theta}(x) d x=1$. We call $f_{\theta}$ the "tilted measure" of $X$, or Esscher transform of $f$. Note that if $K(\theta)=\ln (M(\theta))$ then $f_{\theta}(x)=f(x) e^{\theta x-K(\theta)}$.

When $X$ is Gaussian, its tilted measure is simply the measure of $X$ shifted with a new mean $\theta$.

\section{I.1.1 Example with a process: $X$ is a Brownian motion at time $t$}

Let $X=W_{t}$ be a Brownian motion at time $t$. Then $M(\theta)=e^{\frac{\theta^{2}}{2} t}$ and $f_{\theta}(x)=f(x) e^{\theta x-\frac{\theta^{2}}{2} t}$. We guess immediately the analogy with the Girsanov theorem: $e^{\theta x-\frac{\theta^{2}}{2} t}$ is the density of the Radon-Nykodim derivative from the probability measure $Q$ to the probability measure $\hat{Q}$, under which $\hat{W}_{t}=W_{t}-\theta t$ is a Brownian motion. As we have $\frac{d \hat{Q}}{d Q}{ }_{F_{t}}=e^{\theta W_{t}-\frac{\theta^{2}}{2} t}$ and by applying Bayes' rule:

$$
E^{\hat{Q}}\left[\phi\left(W_{t}\right)\right]=E^{Q}\left[\phi\left(W_{t}\right) \frac{d \hat{Q}}{d Q}\right]=\int \phi(x) f(x) e^{\theta x-\frac{\theta^{2}}{2} t} d x=\int \phi(x) f_{\theta}(x) d x .
$$

But by Girsanov theorem, we also know that:

$$
E^{\hat{Q}}\left[\phi\left(W_{t}\right)\right]=E^{\hat{Q}}\left[\phi\left(\hat{W}_{t}+\theta t\right)\right]=E^{Q}\left[\phi\left(W_{t}+\theta t\right)\right]
$$

as both $W_{t}$ and $\hat{W}_{t}$ are Brownian motions under their respective measures.

So finally:

$$
E^{Q}\left[\phi\left(W_{t}+\theta t\right)\right]=\int \phi(x) f_{\theta}(x) d x
$$

We conclude that $f_{\theta}(x)$ is the density of the translated Brownian motion $W_{t}+\theta t$, with mean $\theta t$. So $f_{\theta}(x)$ is the measure of the original process translated with $\theta t$. Transforming the process into a translated one is also similar to sampling when dealing with Monte Carlo methods. We will see that the application to multivariate distribution of the tilted measure turns out to be also a kind of importance sampling for the $N^{\text {th }}$ to default or the Loss process. 


\section{I.1.2 Example with a non-continuous variable : $X$ is a binomial distribution}

Let $X$ be a binomial distribution with $p=Q(X=1)$. Then we have the following relations:

$$
\begin{aligned}
f(x) & =P(X=x)=p^{x}(1-p)^{1-x} \\
M(\theta) & =E\left[e^{\theta X}\right]=1-p+p e^{\theta}
\end{aligned}
$$

and the tilted measure is:

$$
f_{\theta}(x)=\frac{e^{\theta x} p^{x}(1-p)^{1-x}}{1-p+p e^{\theta}}=\left(\frac{p e^{\theta}}{1-p+p e^{\theta}}\right)^{x}\left(1-\frac{p e^{\theta}}{1-p+p e^{\theta}}\right)^{1-x}=\left(p^{\theta}\right)^{x}\left(1-p^{\theta}\right)^{1-x} .
$$

In other words, the tilted measure is the measure of a binomial distribution with parameter $p^{\theta}=\frac{p e^{\theta}}{1-p+p e^{\theta}}$. Note that $p^{\theta}$ spans $] 0,1[$ as $\theta$ spans $]-\infty,+\infty\left[\right.$ and $p^{\theta=0}=p$. In our applications, $p$ is close to $\lambda T$ with $T$ a year fraction and $\lambda$ the default intensity. So for $\lambda=100 b p s$ then $p=1 \%$. As we can see, $\theta=5$ is enough to transform $p$ to $p^{\theta}=0,5$.

\section{I.1.3 Example with a non-continuous variable : $X$ is a multinomial distribution}

Let now $X=\sum_{i=1}^{N} X_{i}$ with $X_{i}$ a binomial distribution where $Q\left(X_{i}=1\right)=p_{i}$. Thanks to the last example we have:

$$
M(\theta)=E\left[e^{\theta X}\right]=\prod_{i=1}^{N} E\left[e^{\theta X_{i}}\right]=\prod_{i=1}^{N} M_{i}(\theta)
$$

and

$$
K(\theta)=\sum_{i=1}^{N} \ln M_{i}(\theta)=\sum_{i=1}^{N} \ln \left(1-p_{i}+p_{i} e^{\theta}\right)=\sum_{i=1}^{N} K_{i}(\theta) .
$$

The tilted measure applied to $X$ is the measure of a random variable $X^{\theta}$. More precisely, for any measurable function $h$ we have:

$$
\begin{aligned}
E\left[h\left(X^{\theta}\right)\right] & =\int_{\left(x_{1}, \ldots, x_{N}\right) \in\{0,1\}^{N}} h\left(x_{1}+\ldots+x_{N}\right) e^{\theta\left(x_{1}+\ldots+x_{N}\right)-K(\theta)} \prod_{i=1}^{N} f_{i}\left(x_{i}\right) d x_{i} \\
& =\int_{\left(x_{1}, \ldots, x_{N}\right) \in\{0,1\}^{N}} h\left(x_{1}+\ldots+x_{N}\right) \prod_{i=1}^{N} e^{\theta x_{i}} \frac{f_{i}\left(x_{i}\right)}{M_{i}(\theta)} d x_{i} \\
& =\int_{\left(x_{1}, \ldots, x_{N}\right) \in\{0,1\}^{N}} h\left(x_{1}+\ldots+x_{N}\right) \prod_{i=1}^{N} f_{i}^{\theta}\left(x_{i}\right) d x_{i}
\end{aligned}
$$

with

$$
f_{i}^{\theta}\left(x_{i}\right)=\left(p_{i}^{\theta}\right)^{x_{i}}\left(1-p_{i}^{\theta}\right)^{1-x_{i}} \text { and } p_{i}^{\theta}=\frac{p_{i} e^{\theta}}{1-p_{i}+p_{i} e^{\theta}} .
$$

So we see that the tilted measure of $X$ is a multinomial distribution associated with $\left(p_{i}^{\theta}\right)_{i=1, N}$. Then applying the tilted measure on $X$ is surprisingly equivalent to applying it individually to each $X_{i}$. This is quite 
remarkable and comes from the dependence of the $X_{i}$. Note that we have:

$$
E[X]=\sum_{i=1}^{N} p_{i} \text { and } E\left[X^{\theta}\right]=\sum_{i=1}^{N} p_{i}^{\theta}
$$

and if we define:

$$
\eta_{k}=Q(X=k) \text { and } \eta_{k}^{\theta}=Q\left(X^{\theta}=k\right)
$$

then

$$
\eta_{k}=\eta_{k}^{\theta} \cdot e^{K(\theta)-\theta k}
$$

Let define our shift by fixing an arbitrary mean $m_{0}$ and search for $\hat{\theta}$ such that $\sum_{i=1}^{N} p_{i}^{\hat{\theta}}=m_{0}$.

Then $\hat{\theta}$ is called Saddle-point associated to the "new mean" $m_{0}$ because $E\left[X^{\theta}\right]=m_{0}$. The transformation from the distribution $\left(p_{i}\right)_{i=1, N}$ to the distribution $\left(p_{i}^{\hat{\theta}}\right)_{i=1, N}$ is called "Esscher Transform" (cf. [12]):

$$
K^{\prime}(\hat{\theta})=\sum_{i=1}^{N} p_{i}^{\hat{\theta}}=\sum_{i=1}^{N} \frac{p_{i} e^{\hat{\theta}}}{1-p_{i}+p_{i} e^{\hat{\theta}}}=m_{0}
$$

The new distribution is not centered at the initial $E[X]$ but at $m_{0}$. Note that $K^{\prime}(-\infty)=0_{+}$and that $K^{\prime}(+\infty)=\tilde{N}$ where $\tilde{N}$ is the number of $p_{i}$ strictly positive $p_{i}$.Said differently, $\tilde{N}$ is the maximum number of defaults that can occur in the portfolio, and $K^{\prime}(\hat{\theta})$ is always smaller or equal to that number. This remark is important as in the computation of tail probabilities for CDO portfolio, because it can happen that the conditioning on a state variable $Z$ some $p_{i}^{z}$ may be null.

As a conclusion, we have seen through 3 examples that the Esscher Transform does "not modify the nature of the random variable, but just modify its mean" (cf. [13]).

\section{I.2 Application to the pricing of a $N^{\text {th }}$ to default swap, using FFT method}

In a credit derivatives basket, the number of names $n$ is typical around 125 or more for CDOs and much smaller for $m_{0}^{\text {th }}$-to-defaults. The expected number of defaults implied for the credit curves is usually below 5. So computing the fair spread of a $m_{0}^{\text {th }}$-to-default tranche for $m_{0}$ greater than 5 will usually turn into numerical imprecision as we reach the machine precision of $10^{-16}$. This is a problem that often happens when one wants to compute the "tail probabilities". So shifting the counting process mean to a higher mean will remove this problem.

Let suppose that we want to value a $m_{0}^{\text {th }}$-to-default swap and $m_{0}$ is greater that the expected number of defaults.

In order to compute the fair spread of a $m_{0}^{\text {th }}$ to default swap, we need to compute its fixed leg and its protection leg. We assume that both of those legs expected values are only function of the discount factors and the survival probabilities of the $m_{0}^{t h}$-to-default event. Said differently, we only need to compute

$$
Q\left(X(t)<m_{0}\right)=\kappa_{0}(t)+\ldots+\kappa_{m_{0}-1}(t)=1-Q\left(X(t) \geq m_{0}\right) \text { and } \kappa_{k}(t)=Q(X(t)=k)
$$

so we actually only need to compute the tail $Q\left(X(t) \geq m_{0}\right)$. 
Using the third example in the first part " $X$ is a multinomial distribution", we first have to find $\hat{\theta}$ such that

$$
\sum_{i=1}^{n} p_{i}^{\hat{\theta}}=m_{0} \text { with } p_{i}^{\hat{\theta}}=\frac{p_{i} e^{\hat{\theta}}}{1-p_{i}+p_{i} e^{\hat{\theta}}} \text { and } p_{i}=Q\left(\tau_{i} \leq t\right) .
$$

In other words, we shift the mean of the distribution of $X(t)$ to be exactly at $m_{0}$. We find easily $\hat{\theta}$ using a Newton Raphson algorithm. Using the FFT method, we compute $\eta_{k}^{\theta}(t)=Q\left(X^{\hat{\theta}}(t)=k\right)$ for this transformed $X^{\theta}(t)$. Finally we back out $\kappa_{k}(t)$ using $\kappa_{k}(t)=\kappa_{k}^{\theta}(t) \cdot e^{\hat{\theta} k-K(\hat{\theta})}$.

As the names are independent conditional on the latent variable $Z=z$ we have the survival probability of the $n^{\text {th }}$ tho default basket given by :

$$
Q\left(X(t) \geq m_{0}\right)=\int_{-\infty}^{+\infty} Q\left(X^{Z}(t) \geq m_{0}\right) \phi(z) d z
$$

where $Q\left(X^{Z}(t) \geq m_{0}\right)$ is computed using independent $X_{i}^{Z}$.

\section{References}

[1] L. Andersen, J. Sidenius, S. Basu "All your hedges in one basket", Risk November 2003.

[2] L. Andersen, J. Sidenius "Extensions to the Gaussian copula: random recovery and random factor loadings", 2005 Journal of Credit Risk, 1(1), 29-70.

[3] A. Antonov, S. Mechkov, T. Misirpashaev"Analytical Techniques for Synthetic CDOs and Credit Default Risk Measures", Numerix working paper 2005.

[4] C. Corrado, T. Su "Skewnness and Kurtosis in S\&P500 index returns implied by option prices" 1996 The Journal Of Financial Research 19(2) p.175-192.

[5] H. Cramer "mathematical methods of Statistics" , 1946 Princeton University Press.

[6] H. Daniels "Tail Probability Approximations", 1987 International Statistical Review 55 37-48

[7] H. Daniels "Saddle-point Approximations In Statistics", 1954, Ann. Math. Statist. 25 631-650.

[8] J. Yang, T. Hurd, X. Zhang "Saddle-point Approximation Method for Pricing CDOs", working paper 2005

[9] M. Akahira, K. Takahashi "A Higher Order Large Deviation Approximation for The Discrete Distributions", J. Japan Stat. Soc. Vol 31 No.2 2001 257-267

[10] H. Bäuhlmann, F. Delbean, P. Embrechts, A.N. Shiryaev "On Esscher Transform in Discrete Finance Models", ASTIN papers

[11] Y. Elouerkhaoui "Credit Derivatives : the Homogeneous Expansion", UBS working paper 2002

[12] F. Esscher "On the Probability Function in the Collective Theory of Risk", Skandinavisk Aktuarietidskrift 151932 175-95 
[13] H. Geman, Transaction of Society of Actuaries 1994 Vol.46

[14] H. Geman, N. El Karoui, J.C. Rochet "Changes of Numeraire, Change of Probability Measures and Option Pricing", AFFI conferenc Paris 1992

[15] H.U. Gerber, E.S.W. Shiu "Option Pricing By Esscher Transform", Transaction of Society of Actuaries 1994 Vol.46

[16] R. Jarrow, A. Rudd "Approximate Option Valuation For Arbitrary Stochastic Processes", 1982 Journal of Financial Economics 101982 347-369.

[17] JP Morgan Credit Research Team "Introducing base correlation", JP Morgan Credit Derivatives Strategy March 2004

[18] J.L. Jensen "Saddle-point Approximations", Oxford Statistical Science Series 16

[19] S. Kendall "The advanced theory of statistics", vol1 : distribution theory (Macmillan, NY)

[20] D. Li "On default Correlation : a copula function approach", Risk Metrics group 2000

[21] T. Roncalli " modelling dependence in finance using copulas", Credit Lyonnais GRO 2001

[22] R.B. Nelsen "An introduction to copulas", Springer Verlag 1998

[23] J.P. Laurent, J gregory "basket default swap, CDO's and Factor Copulas", 2002 working paper

[24] R. Lugannani, S. Rice "Saddle-point approximations for the distribution of the sum of independent random variables", Advances in Applied Probability 12: 4754901980

[25] R. Martin, K. Thompson , C. Browne "Taking to the Saddle", Risk June 2001

[26] D. Shelton "Back To Normal", Citigroup Research avalaible on www.defaultrisk.com

[27] J.Turc P. Very B. Benhamou "Pricing CDOs with a Smile", Societe Generale Quantitative Strategy February 2005

[28] D. Taras, C. Cloke-Browne, E. Kalimtgis "Analytical improvment of the Saddle-point approximation and spread risk attribution in a portfolio of tranches", 2005 www.defaultrisk.com

[29] L. Veilex "A Relative Value Approach For CDO Pricing", Goldman Sachs working paper 2004

[30] J. Yang, T. Hurd, X. Zhang "Saddle-point approximation method for pricing", working paper 2005 www.defaultrisk.com 